\title{
CROSS SECTION EVALUATION WORKING GROUP BENCHMARK SPECIFICATIONS
}

\author{
VOLUME II \\ SUPPLEMENT
}

September 1986

\author{
NATIONAL NUCLEAR DATA CENTER \\ BROOKHAVEN NATIONAL LABORATORY \\ UPTON, LONG ISLAND, NEW YORK 11973 U.S.A.
}

\author{
BROOKHAVEN NATIONAL LABORATORY \\ ASSOCIATED UNIVERSITIES, INC. \\ UNDER CONTRACT NO. DE-AC02-76CH00016 WITH THE \\ UNITED STATES DEPARTMENT OF ENERGY
}




\section{DISCLAIMER}

This report was prepared as an account of work sponsored by an agency of the United States Government. Neither the United States Government nor any agency thereof, nor any of their employees, nor any of their contractors, subcontractors, or their employees, makes any warranty, express or implied, or assumes any legal liability or responsibility for the accuracy, completeness, or usefulness of any information, apparatus, product, or process disclosed, or represents that its use would not infringe privately owned rights. Reference herein to any specific commercial product, process, or service by trade name, trademark, manufacturer, or otherwise, does not necessarily constitute or imply its endorsement, recommendation, or favoring by the United States Government or any agency, contractor or subcontractor thereof. The views and opinions of authors expressed herein do not necessarily state or reflect those of the United States Government or any agency, contractor or subcontractor thereof.

Printed in the United States of America

Available from

National Technical Information Service

U.S. Department of Commerce

5285 Port Royal Road

Springfield, VA 22161

NTIS price codes:

Printed Copy: A04; Microfiche Copy: A01 
TABLE OF CONTENTS

Section

$\underline{\text { Page }}$

I. Introduction

A. Availability of Integral Experiment Information (The SDT and SB Series)

B. Relevance of the Benchmarks

II. Compilation of Shielding Benchmark Specifications

SB5. Fusion Reactor Shielding Benchmark

SB6. Fusion Reactor Shielding Benchmark II. Duct Streaming Experiment and Analyses 
A. Benchmark Name and Type: Fusion Rector Shielding Benchmark II: Duct Streaming Experiments and Analyses.

B. System Description: Neutron and photon flux spectra have been measured and calculated for the case of neutrons produced by $D-T$ reactions streaming through a cylindrical iron duct surrounded by concrete. Measurements and calculations have also been obtained when the iron duct is partially filled by a laminated stainless steel and borated polyethylene shadow bar.

C. Model Description: The experimental configuration is threedimensional but may to a good approximation be reduced to a two-dimensional configuration. The three-dimensional configuration will first be described and then the two-dimensional configuration used in the ORNL analysis will be discussed. Both the three-dimensional configuration and the approximate twodimensional configuration are similar to those used in the benchmark SB5. 1

\section{Three-Dimensional Configuration}

In Fig. 1 a horizontal cut through the room that houses the experiment is given. In this figure and throughout this discussion the z-axis is the axis of the deuteron beam, the $x$-axis is parallel to the floor and roof, and the $y$-axis is perpendicular to the floor and the roof. The iron cavity liner around the target serves as a spectrum modifier and makes the neutron spectrum incident on the experimental shield configuration similar to the spectrum incident on the first wall of a fusion reactor. The thermal neutron shield in Figure 1 is composed of stainless steel 304, is $5.08 \mathrm{~cm}$ thick, and has a square cross sectional area, $152.4 \mathrm{~cm}$ by $152.4 \mathrm{~cm}$. In Figs. 2 and 3 other views of the experimental enclosure and the experimental room are given. 
In the present series of experiments the spatial region labeled "the experimental shield configuration", Fig. 1 was filled by a concrete block with a cylindrical iron duct at its center. The concrete block has a height of $172.72 \mathrm{~cm}$, a width of $156.2 \mathrm{~cm}$ and a length of $78.0 \mathrm{~cm}$. The iron duct has an inside diameter of $30.0 \mathrm{~cm}$, a thickness of $7.50 \mathrm{~cm}$. The iron duct forms an extension of the source can. The duct extends $85.0 \mathrm{~cm}$ from the tritium target ' (neutron source) and the length-to-diameter (L/D) ratio of the duct is 2.83. The $L / D$ ratio is measured from the tritium target to the mouth of the duct.

The dimensions given in Figs. $1-3$ and above are sufficient to enable the experiment to be modeled to any desired detail, but the reader who wishes to model the experiment in three dimensions should also see Ref. 2. The positions of the detectors in Figs. 1 and 3 are only illustrative. The detector positions at which experimental data are available, are completely defined in Section D.

\section{Two-Dimensional Configuration}

In Fig. 4 the reduced geometry representation of the experiment that is used to obtain the calculated results presented later is shown. In some cases the iron duct was partially filled by a cylindrical shadow. In Fig. 5 the reduced geometry of the experiment is shown with the shadow bar in place. Also, in Fig. 5, the dimensions and composition of the shadow bar are shown. The end of the shadow bar closest to the source is at $z=77.0 \mathrm{~cm}$.

The geometry in Figs. 4 and 5 is two-dimensional in that there is cylindrical symmetry about the $z$ axis. The concrete block around the duct has a radius of $72.5 \mathrm{~cm}$. In the two-dimensional model the lithiated paraffin that is shown in Fig. $l$ is not included. Also in the two-dimensional model the back concrete wall is placed directly against the stainless steel thermal shield.

In the two-dimensional model the radial boundary of the configuration is taken as an albedo surface with a reflection coefficient of 0.2 for all particle types and all energies. This reflection coefficient was determined by matching particle flux contours obtained with the two-dimensional 


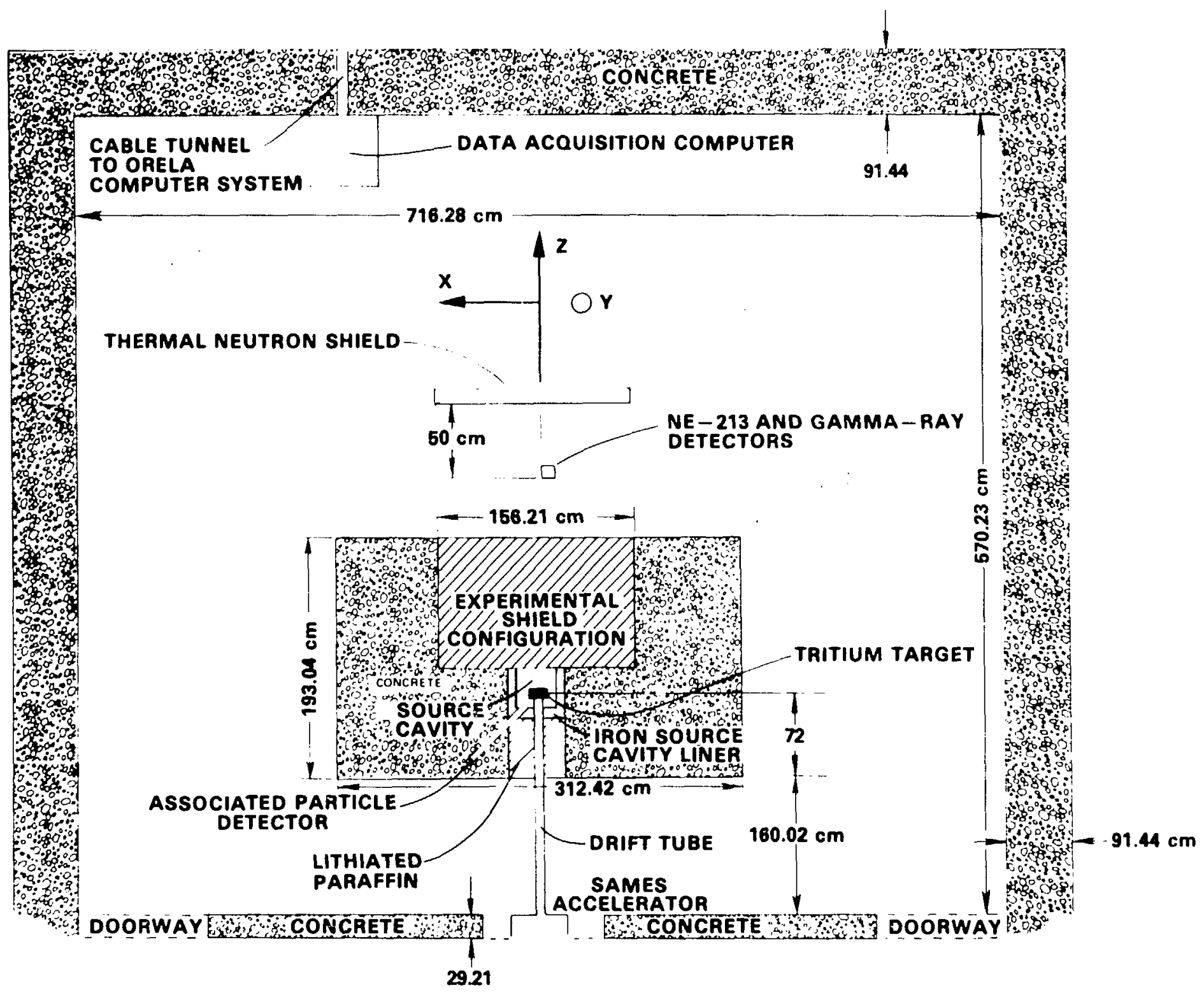

Fig. 1. Horizontal schematic of experimental enclosure. See Figs. 2 and 3 for additional dimensions. [Drawing not to scale] 


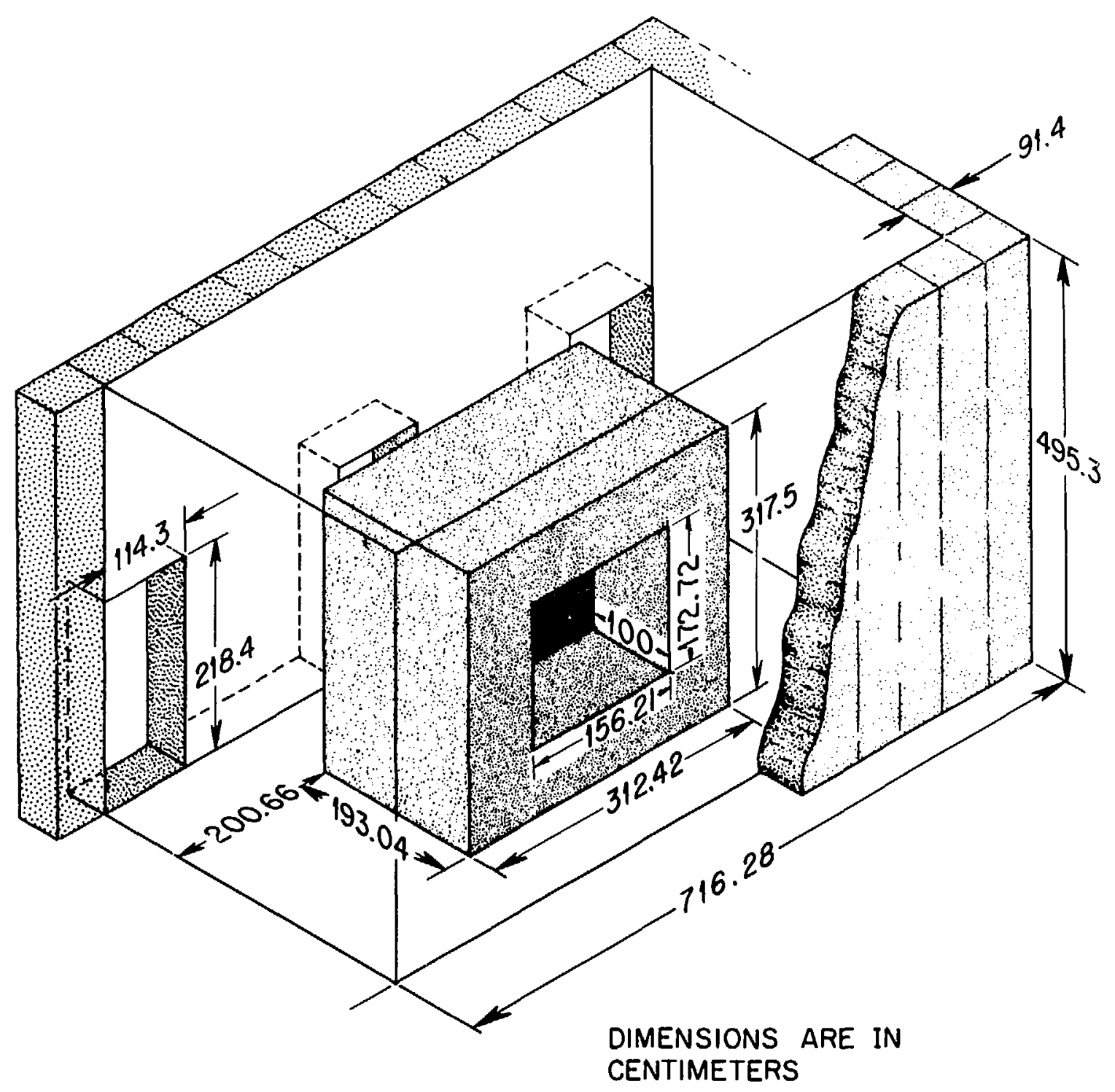

Fig. 2. Three-dimensional view of experimental enclosure. See Figs. 1 and 3 for additional dimensions. [Drawing not to scale] 
FIGURE 3

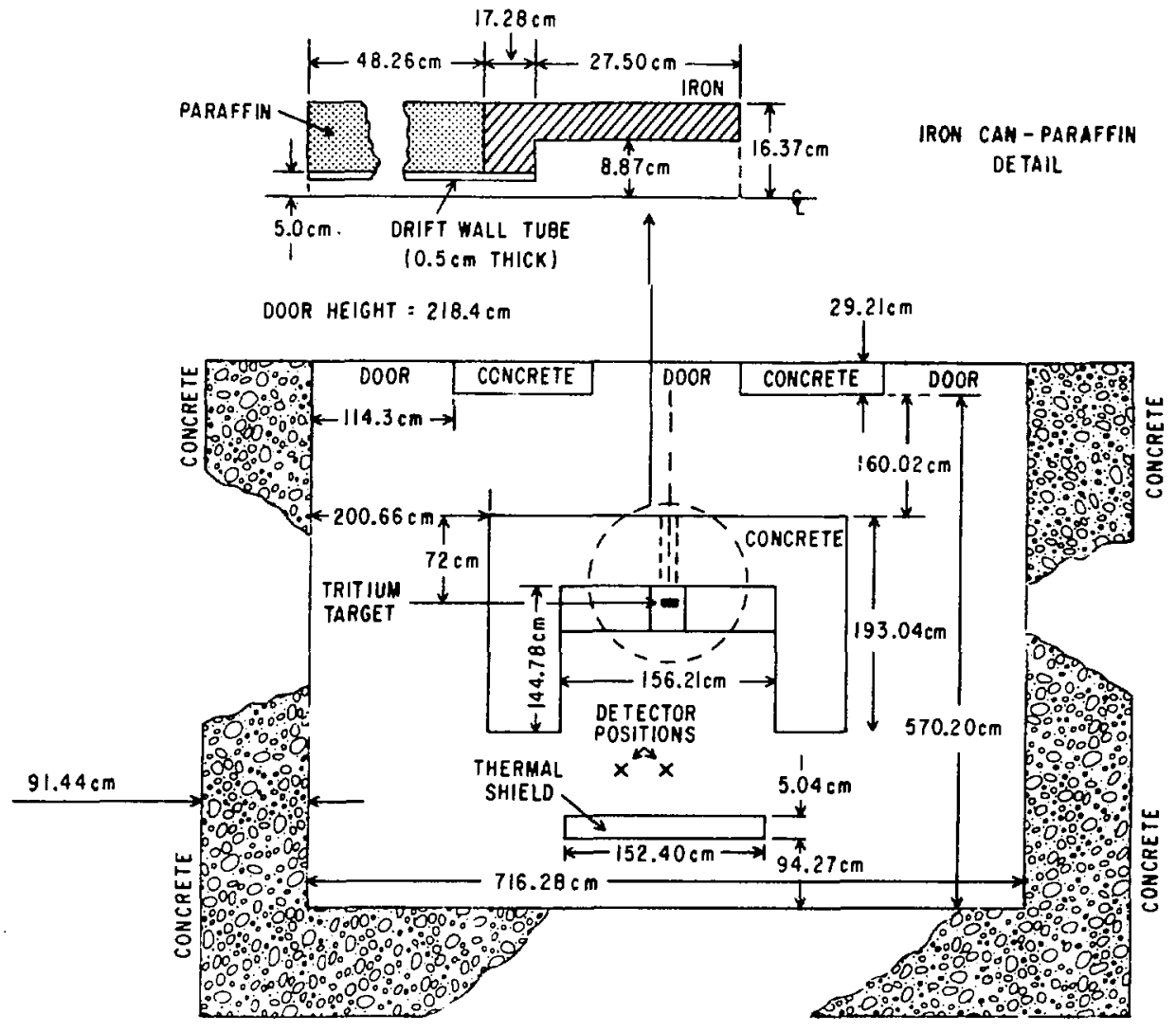

HORIZONTAL SLICE OF MAGNETIC FUSION ENERGY (MFE) SHIELO AND ROOM

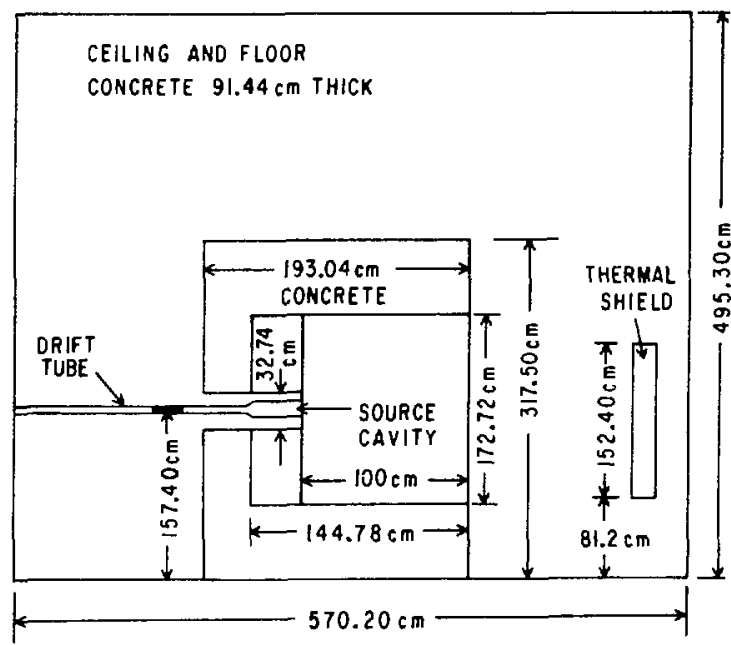

VERTICAL SLICE OF MFE SHIELD AND ROOM

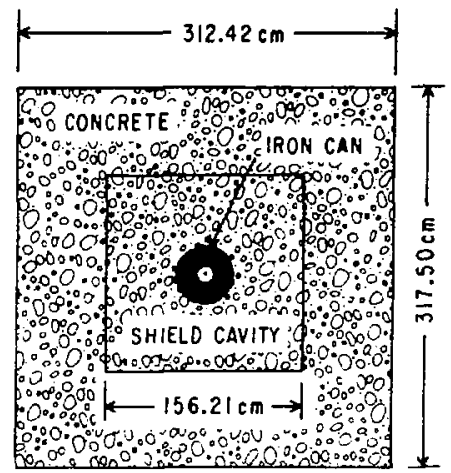

FRONT VIEW OF SOURCE CAN AND SHIELD

Fig. 3. Additional schematics of experimental enclosure. See Figs. 1 and 2 for additional dimensions. [Drawing not to scale] 


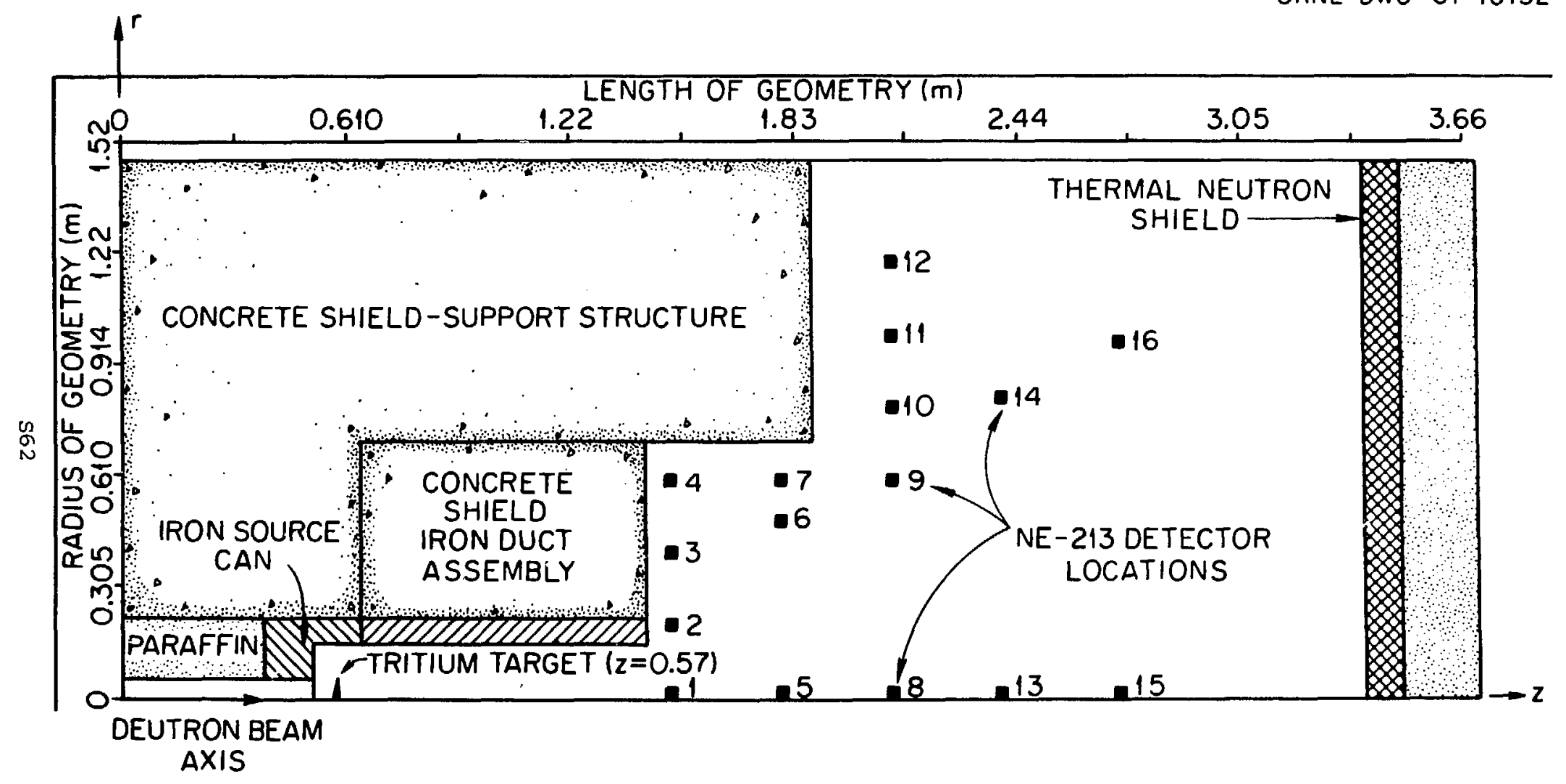

Fig. 4. Two-dimensional model of the experimental configuration. The model is cylindrically symmetric about the deuteron beam axis. 


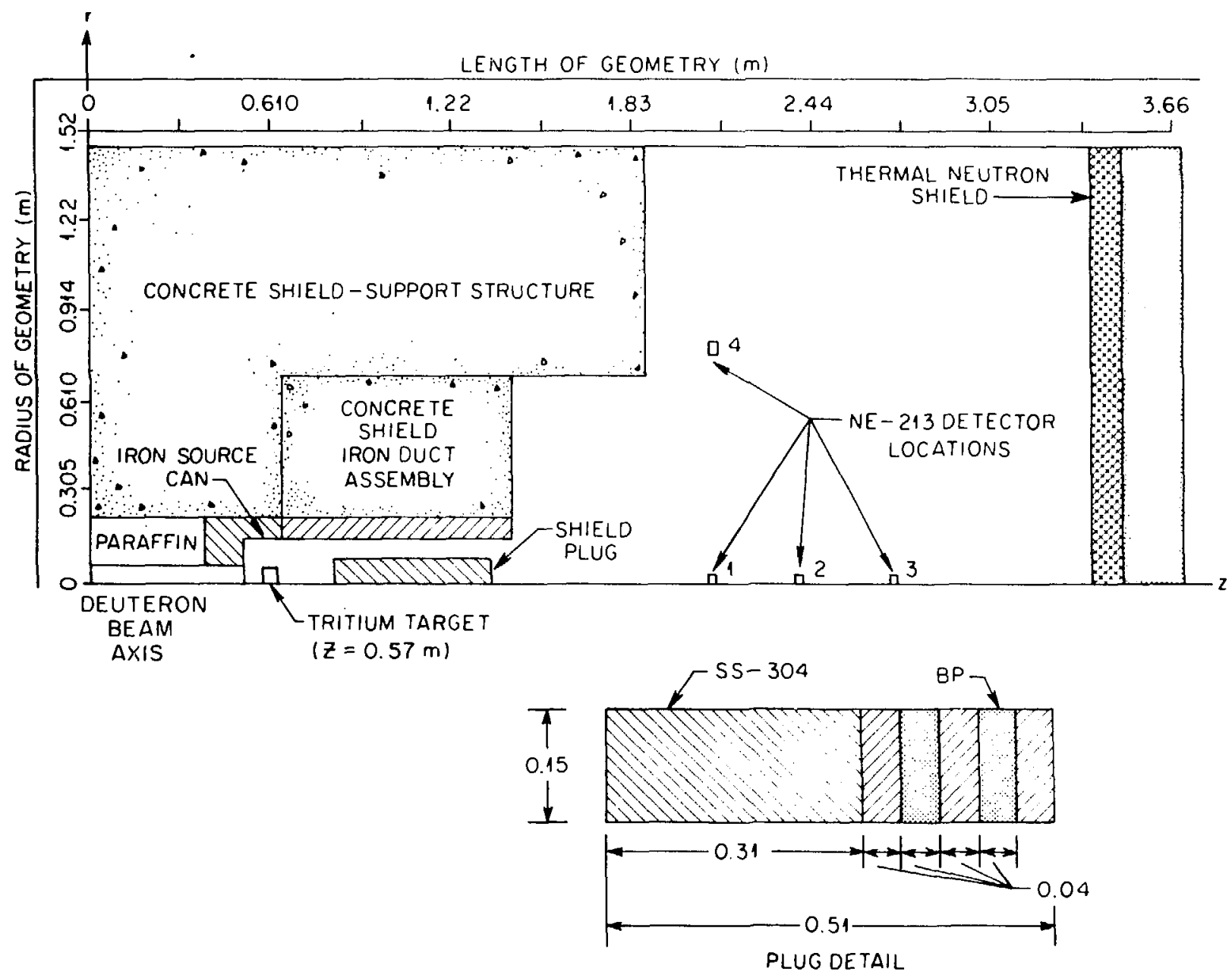

Fig. 5. Two-dimensional model of the experimental configuration with the shield plug, i.e., shadow bar, in place. 
representation of the complete experimental room. More details concerning the determination of this reflection coefficient will be found in Ref. 2 .

\section{Material Composition}

In Table 1 the number densities of the materials that occur in Figs. 1-5 are given. These are the number densities that were used in carrying out all of the calculation.

4. Method of Calculation

With the two-dimensional model the benchmark may be calculated with either discrete ordinates or Monte Carlo methods. In the case of discrete ordinates methods, however, satisfactory results are not obtained until corrections obtained using Monte Carlo methods are applied. The calculational procedures are described and the results obtained are presented in Section $E$, Analytical Results and Comparisons with Experimental Data.

The benchmark may also be calculated using a thret-dimensional model and Monte Carlo techniques, but such calculations have not yet been performed.

D. Experimental Data: The neutron source was obtained by focusing a 250 keV deuteron beam on a cylindrical titanium-tritide (TiT) target having a radius of $0.64 \mathrm{~cm}$, a thickness of $4 \mathrm{mg} / \mathrm{cm}^{3}$ and a density of $4.28 \mathrm{~g} / \mathrm{cm}^{3}$. The neutron source strength was measured by using the associated particle technique.

The neutron-gamma-ray detector consisted of $66.1 \mathrm{~g}$ of $\mathrm{NE}-213$ 1iquid scintillator contained in a cylindrical aluminum can having a wall thickness of $4.32 \times 10^{-2} \mathrm{~cm}$. The active volume of the detector was $79 \mathrm{~cm}^{3}(4.66 \mathrm{~cm}$ diameter and $4.66 \mathrm{~cm}$ height). Neutron and gamma-ray events were separated using pulse shape discrimination methods. The neutron and gamma pulse height spectra were unfolded using FERD ${ }^{4}$ to produce energy spectra. The neutron response matrix was obtained using the pulsed neutron beam from the Oak Ridge Electron Linear Accelerator and the gamma-ray response matrix was generated using gamma-ray sources of known energy. The energy resolution of the 
detector varies as

$$
R_{N}=\sqrt{300+\frac{800}{E_{N}}}
$$

for neutrons of energy $\mathrm{E}_{\mathrm{N}}$ in $\mathrm{MeV}$, and

$$
R \gamma=\sqrt{130+\frac{288}{B \gamma}}
$$

for gamma rays of energy $E_{\gamma}$ in $M e V$. The quantities $R_{N}$ and $R_{\gamma}$ are the full width at half maximum (in percent) of the detector response (assumed Gaussian) to neutrons and gamma rays.

Experimental data have been obtained at 16 detector locations when the shadow bar was not present and at 4 detector locations when the shadow bar was present. In the experiment, the detector position was specified in a right-handed coordinate system with the $z$ axis corresponding to the deuteron beam axis and the $x$ axis parallel to the experimental room floor. The coordinates of the detector positions at which experimental data are available are given in Table 2. The origin of $z$ coordinates is as shown in Figs. 4 and 5. In this coordinate system the target is at $z=0.57 \mathrm{~m}$ so the source to detector distance is obtained by subtracting $0.57 \mathrm{~m}$ from the $z$ coordinate of the detector given in Table 2. The detector positions are shown in Figs. 4 and 5. In the two dimensional models the radial coordinates of the detector are given by $\sqrt{\mathrm{x}^{2}+\mathrm{y}^{2}}$.

At each detector location the neutron flux per unit energy for all energies $\geqslant 850 \mathrm{keV}$ and the gamma-ray $f 1 \mathrm{ux}$ per unit energy for all energies $>750$ $\mathrm{keV}$ have been measured. The experimental data at each detector location are given in Appendix A. As part of the data unfolding process, a confidence interval with a width of two standard deviations is obtained so the data presented are in all cases the upper and lower limits of this confidence interval. 
Table 1. Composition of Materials Used in the Calculation

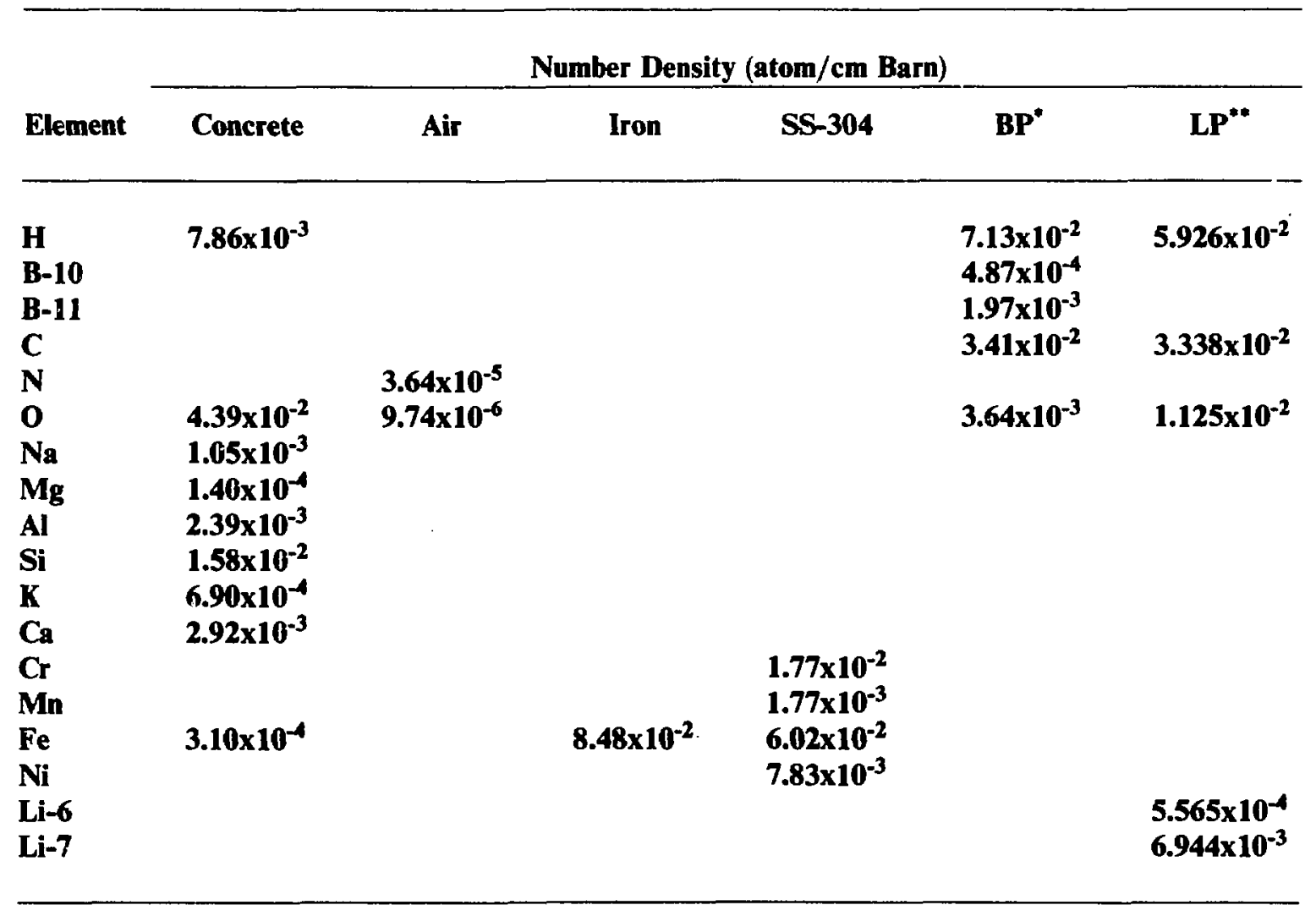

"BP = Borated Polyethylene

"LP = Lithisted Paraffin 
E. Analytical Results and Comparisons with Experimental Data: All of the analytical results reported in this benchmark description are based on the two-dimensional configurations shown in Figs. 4 and 5.

In the three subsections below, analytice? results obtained using discrete ordinates methods, discrete ordinates plus Monte Carlo methods, and Monte C'arlo methods are presented and compared with the experimental data. Only representative comparisons are presented here but comparisons with all of the data are given in Refs. 5, 6, and 7 .

\section{Discrete Ordinates Transport Methods}

The sequence of radiation transport calculations used to obtain neutron and gamma-ray spectra by discrete ordinates methods is "complex and has been described in detail elsewhere so only a very brief description will be given here. $1,2,5,6,7$

The configuration in Fig. 4 was modeled in $r-z$ geometry using 62 radial and 87 axial mesh intervals. The radial and axial boundaries used in the calculations are given in Table 3. The energy-angle dependence of the neutrons from the $D-T$ source was taken into account in the calculations by the methods described in Refs. 1, 2, 5, 6, and 7. The cross section data used are based on ENDF/B-IV, are the same as those used in Ref. 1, and are available from the Radiation Shielding Information Center of the Oak Ridge National Laboratory. 8 The group boundaries are shown in Table 4. The calculations were carried out using the $\operatorname{DOT}^{9}$ code in conjunction with the first and last flight codes GRTUNCL ${ }^{10}$ and FALSTF ${ }^{11}$. In the calculations, a $S_{12}$ angular quadrature and a $\mathrm{P}_{3}$ Legendre expansion were used. A flux convergence criterion of $10^{-4}$ was used and the flux was calculated using weighted differences. Here, as in Ref. 1, the radial boundary of the two-dimensional configuration was taken to be an albedo surface for both neutron and gamma rays, with a reflection coefficient of 0.20 at all energies. The manner in which this reflection coefficient was'derived is described in Ref. 2 .

In Fig. 6 the calculated and experimental neutron fluxes per unit 
Table 2. Detector Coordinates

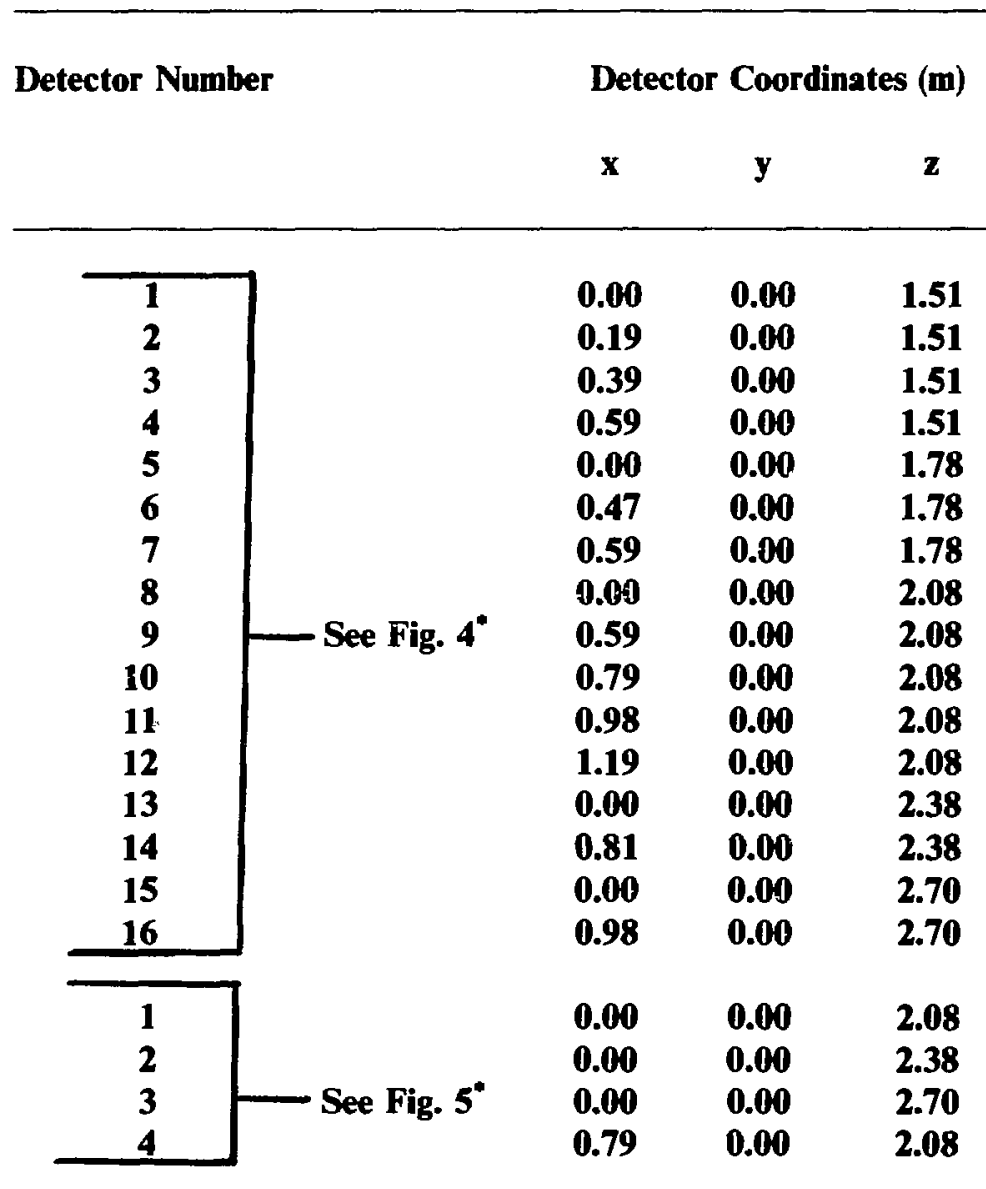

Figs. 4 and 5 show a two-dimensional model of the experimental configuration, so in Figs. 4 and 5 the radial position, $r$, of the detector is given by $\sqrt{x^{2}+y^{2}}$. 
Table 3. The Radial and Axial Boundaries Used in the Two-Dimensional Model of the Experiment

Radial Boundaries

(cm)
Axial Boundaries

(cm)

\begin{tabular}{|c|c|c|c|}
\hline 0 & 6.0000E 01 & $1.5000 \mathrm{E} 02$ & 2.9498E 02 \\
\hline $5.0000 \mathrm{E}-01$ & 6.1000E 01 & 1.5750E 02 & 2.9798E 02 \\
\hline 1.0000E 00 & $6.4000 \mathrm{E} 01$ & $1.6500 \mathrm{E} 02$ & 2.9898E 02 \\
\hline 2.0000E 00 & 6.7000E 01 & 1.7250E 02 & 2.9998E 02 \\
\hline 3.0000E O0 & 7.0000E 01 & 1.8000E 02 & $3.0348 \mathrm{E} 02$ \\
\hline 4.0000E 00 & 7.3000E 01 & 1.8750E 02 & $3.0698 \mathrm{E} 02$ \\
\hline $5.0000 \mathrm{E} 00$ & $7.6000 \mathrm{E} 01$ & 1.9500E 02 & $3.1048 E 02$ \\
\hline $7.5000 \mathrm{E} 00$ & 7.9000E 01 & $1.9650 \mathrm{E} 02$ & 3.1398E 02 \\
\hline 1.0000E 01 & 8.0000E 01 & 1.9800E 02 & 3.1748E 02 \\
\hline $1.2500 \mathrm{E} 01$ & 8.1000E 01 & 1.9950E 02 & $3.2098 E 02$ \\
\hline 1.5000E 01 & 8.3833E 01 & $2.0100 \mathrm{E} 02$ & 3.2448E 02 \\
\hline 1.6333E 01 & $8.6667 \mathrm{E} 01$ & 2.0250E 02 & 3.2798E 02 \\
\hline $1.7667 \mathrm{E} 01$ & $8.9500 E 01$ & $2.0350 E 02$ & 3.2898E 02 \\
\hline $1.9000 E 01$ & 9.2333E 01 & $2.0450 \mathrm{E} 02$ & 3.2998E 02 \\
\hline 2.0397E 01 & 9.5167E 01 & 2.0550E 02 & 3.3249E 02 \\
\hline 2.1795E 01 & 9.8000E 01 & $2.0650 \mathrm{E} 02$ & 3.3499E 02 \\
\hline 2.4197E 01 & 9.9500E 01 & $2.0750 \mathrm{E} 02$ & 3.3750E 02 \\
\hline 2.6598E 01 & 1.0100E 02 & $2.0850 \mathrm{E} 02$ & 3.5798E, 02 \\
\hline $2.9000 \mathrm{E} 01$ & $1.0400 \mathrm{E} 02$ & $2.0950 \mathrm{E} 02$ & 3.5898E 02 \\
\hline $3.0000 E 01$ & 1.0700E 02 & $2.1050 E 02$ & 3.5998E 02 \\
\hline $3.1000 \mathrm{E} 01$ & $1.1000 \mathrm{E} 02$ & $2.1150 \mathrm{E} 02$ & $3.6931 \mathrm{E} 02$ \\
\hline $3.3667 \mathrm{E} 01$ & $1.1300 \mathrm{E} 02$ & $2.1250 \mathrm{E} 02$ & 3.7865E 02 \\
\hline $3.6333 \mathrm{E} 01$ & $1.1600 \mathrm{E} 02$ & $2.1400 \mathrm{E} 02$ & $3.8798 \mathrm{E} 02$ \\
\hline $3.9000 \mathrm{E} 01$ & $1.1900 \mathrm{E} 02$ & 2.1407E 02 & 3.8898E 02 \\
\hline $4.0000 E 01$ & $1.2000 \mathrm{E} 02$ & $2.1650 \mathrm{E} 02$ & 3.8998E 02 \\
\hline $4.1000 \mathrm{E} 01$ & $1.2100 \mathrm{E} 02$ & 2.2053E 02 & $3.9998 \mathrm{E} 02$ \\
\hline $4.4000 \mathrm{E} 01$ & $1.2700 \mathrm{E} 02$ & 2.2455E 02 & 4.0998E 02 \\
\hline $4.7000 \mathrm{E} 0 \mathrm{I}$ & $1.4000 \mathrm{E} 02$ & $2.2858 E 02$ & 4.1998E 02 \\
\hline $5.0000 E 01$ & $1.4250 \mathrm{E} 02$ & $2.3261 \mathrm{E} 02$ & 4.2098E 02 \\
\hline $5.3000 \mathrm{E} 01$ & $1.4500 \mathrm{E} 02$ & $2.3663 \mathrm{E} 02$ & $4.2198 \mathrm{E} 02$ \\
\hline $5.6000 \mathrm{E} \mathrm{O1}$ & 1.4750E 02 & $2.4066 \mathrm{E} 02$ & 4.3307E 02 \\
\hline \multirow[t]{13}{*}{$5.9000 \mathrm{E} 01$} & & $2.4469 \mathrm{E} 02$ & 4.4415E 02 \\
\hline & & 2.4871E 02 & $4.5524 E 02$ \\
\hline & & $2.5274 \mathrm{E} 02$ & 4.6633E 02 \\
\hline & & $2.5677 \mathrm{E} 02$ & $4.7741 \mathrm{E} 02$ \\
\hline & & 2.6079E 02 & 4.8850E 02 \\
\hline & & $2.6482 E 02$ & 4.9100E 02 \\
\hline & & 2.6885E 02 & 4.9350E 02 \\
\hline & & 2.7287E 02 & 4.9600E 02 \\
\hline & & $2.7690 \mathrm{E} 02$ & 4.9850E 02 \\
\hline & & 2.8093E 02 & 5.0375E 02 \\
\hline & & 2.8495E 02 & 5.0900E 02 \\
\hline & & 2.8898E 02 & 5.1426E 02 \\
\hline & & 2.9198E 02 & 5.1950E 02 \\
\hline
\end{tabular}

\footnotetext{
'These axial boundaries have an origin of coordinates that is different from that shown in Figs. 4 and 5 . To express the axial boundaries in the coordinate system with the origin of coordinates as shown in Figs. 4 and 5 , the value $150 \mathrm{~cm}$ must be subtracted from the values given in the table.
} 
Table 4. 53-Neutron, 21-Gamma-Ray Energy Radiation Transport Cross Section Groap Structure

\begin{tabular}{|c|c|c|c|c|c|}
\hline $\begin{array}{l}\text { Neutron } \\
\text { Group }\end{array}$ & $\begin{array}{c}\text { Upper Energy } \\
(e V)\end{array}$ & $\begin{array}{l}\text { Neutron } \\
\text { Group }\end{array}$ & $\begin{array}{c}\text { Upper Energy } \\
(\mathrm{eV})\end{array}$ & $\begin{array}{c}\text { Gamma Ray } \\
\text { Group }\end{array}$ & $\begin{array}{c}\text { Upper } \\
\text { Energy } \\
(\mathrm{MeV})\end{array}$ \\
\hline 1 & $0.17333 E+08$ & 28 & $0.60810 E+06$ & 1 & 14.0 \\
\hline 2 & $0.15683 E+08$ & 29 & $0.49787 E+06$ & 2 & 12.0 \\
\hline 3 & $0.14918 E+08$ & 30 & $0.36883 E+06$ & 3 & 10.0 \\
\hline 4 & $0.14550 E+08$ & 31 & $0.29850 E+06$ & 4 & 8.0 \\
\hline 5 & $0.14191 E+08$ & 32 & $0.29720 E+06$ & 5 & 7.5 \\
\hline 6 & $0.13840 E+08$ & 33 & $0.18316 E+06$ & 6 & 7.0 \\
\hline 7 & $0.13499 E+08$ & 34 & $0.11109 E+06$ & 7 & 6.5 \\
\hline 8 & $0.12840 E+08$ & 35 & $0.67379 E+05$ & 8 & 6.0 \\
\hline 9 & $0.12214 E+08$ & 36 & $0.40868 E+05$ & 9 & 5.5 \\
\hline 10 & $0.11052 E+08$ & 37 & $0.24788 E+05$ & 10 & 5.0 \\
\hline 11 & $0.10000 E+08$ & 38 & $0.23579 E+05$ & 11 & 4.5 \\
\hline 12 & $0.90484 E+07$ & 39 & $0.15034 E+05$ & 12 & 4.0 \\
\hline 13 & $0.81873 E+07$ & 40 & $0.91188 E+04$ & 13 & 3.5 \\
\hline 14 & $0.74082 E+07$ & 41 & $0.55308 E+04$ & 14 & 3.0 \\
\hline 15 & $0.60653 E+07$ & 42 & $0.33546 E+04$ & 15 & 2.5 \\
\hline 16 & $0.49659 \mathrm{E}+07$ & 43 & $0.20347 E+04$ & 16 & 2.0 \\
\hline 17 & $0.40657 E+07$ & 44 & $0.12341 E+04$ & 17 & 1.5 \\
\hline 18 & $0.36788 E-07$ & 45 & $0.74852 E+03$ & 18 & 1.0 \\
\hline 19 & $0.27253 E+07$ & 46 & $0.45400 E+03$ & 19 & 0.60 \\
\hline 20 & $0.23653 E+07$ & 47 & $0.27536 E+03$ & 20 & 0.20 \\
\hline 21 & $0.23069 E+07$ & 48 & $0.16702 E+03$ & 21 & 0.10 \\
\hline 22 & $\mathbf{0 . 2 2 3 1 3 E}+07$ & 49 & $\mathbf{0 . 1 0 1 3 0 E}+03$ & & 0.01 \\
\hline 23 & $0.16530 E+07$ & 50 & $0.61442 E+02$ & & \\
\hline 24 & $0.13534 E+07$ & 51 & $0.37267 E+02$ & & \\
\hline 25 & $0.86294 E+06$ & 52 & $0.10677 E+02$ & & \\
\hline 26 & $0.82085 E+06$ & 53 & $0.41399 E+00$ & & \\
\hline 27 & $0.74274 E+06$ & & 0.10000E - 04 & & \\
\hline
\end{tabular}


energy are shown for detectors 1 to 4 in Fig. 4, i.e., at $z=1.5 \mathrm{~lm}$ and $r=$ $0.0,0.19,0.39$, and $0.59 \mathrm{~m}$. The solid curves are the measured spectra and the points show the calculated results. The two solid curves for each detector location represent the $68 \%$ confidence interval, i.e., plus and minus one standard deviation, in the measured spectra that is introduced by the unfolding procedure used to obtain the neutron spectra from the pulse-height distributions. The calculated data have been smoothed by convoluting the neutron flux per unit energy in each multigroup interval with an energydependent Gaussian response function having a width given by Eq. (1). The calculated and measured spectra are absolutely normalized to a source strength of one D-T neutron. For purposes of displaying the data, some of the spectra have been multiplied by $10^{-n}, n=1,2$, etc. The actual magnitude of these spectra are restored by multiplying by the appropriate factor of $10^{\mathrm{n}}$.

Inspection of Fig. 6 reveals that the calculated and measured spectra are generally in good agreement in the neutron energy range between $850 \mathrm{keV}$ and $\sim 10 \mathrm{MeV}$. The neutrons that contribute to the spectra in this energy range result from inelastic reactions of the source and secondary neutrons in the experimental apparatus or are those which have undergone multiple collisions in transit from the source to the detector.

At neutron energies above $\sim 10 \mathrm{MeV}$, the agreement among the calculated and measured differential neutron energy spectra is less favorable and the agreement varies significantly as a function of detector location. The neutrons that contribute to the spectra in this energy range are either uncollided neutrons emanating directly from the source (particularly for the on-axis detectors) or are those that reach the detector as the result of a single scattering from the experimental apparatus, particularly the iron duct. These single scattered neutrons arise from both elastic and inelastic reactions which at energies above $\sim 10 \mathrm{MeV}$ have angular distributions that are very forward peaked. When these angular distributions are represented by a $\mathrm{P}_{3}$ Legendre expansion, the angular dependence of the scattering probabilities are not adequately described. As a result, the neutron flux at the detector may be either over- or under-estimated depending upon the angle at which the neutron scatters in transit from the source to the detector. This phenomenon 
is evident in the calculated spectra at the off-axis detector locations shown in Fig. 4.

In order to assess the impact of single scattering on the neutron energy spectra, the discrete ordinates calculations were carried out using a $\mathrm{P}_{7}$ Legendre polynomial expansion to represent the neutron scattering cross sections. Although some improvement was noted in the agreement between the calculated and measured spectra, it became apparent that satisfactory comparisons required an even higher order of expansion to account for the single scattered neutron contribution to the high energy reutron flux. Results obtained when the single scattering contribution to the neutron flux is calculated using Monte Carlo methods are presented in the next subsection of this benchmark specification.

The calculated differential gamma-ray spectra are compared with the measured spectra in Fig. 7 for the same four detectors considered in Fig. 6. Similar comparisons for the other detector locations in Fig. 4 are given in Ref. 5. These spectra are compared for gamma rays having energies above 750 keV. The calculated data were obtained by convoluting the gamma-ray flux per unit energy in each multigroup energy interval with an energy-dependent Gaussian response function having a width given by Eq. (2). Al1 of the calculated data were obtained using the discrete ordinates transport methods. Gámma rays produced from inelastic and neutron capture reactions have nearly isotropic angular distributions and are well represented in the discrete ordinates transport calculations by $a P_{3}$ Legendre polynomial expansion. The calculation generally reproduces the measurements in magnitude but because of the rather coarse energy group structure, the various peaks in the measured differential distributions are not reproduced.

2. Discrete Ordinates Plus Monte Carlo Transport Methods

To determine if the discrepancies shown in Fig. 6 are due to an inadequate calculation of the single scattering contribution to the neutron flux, a procedure was developed that allows the single scattering contribution to be calculated by Monte Carlo methods. This procedure is described in 


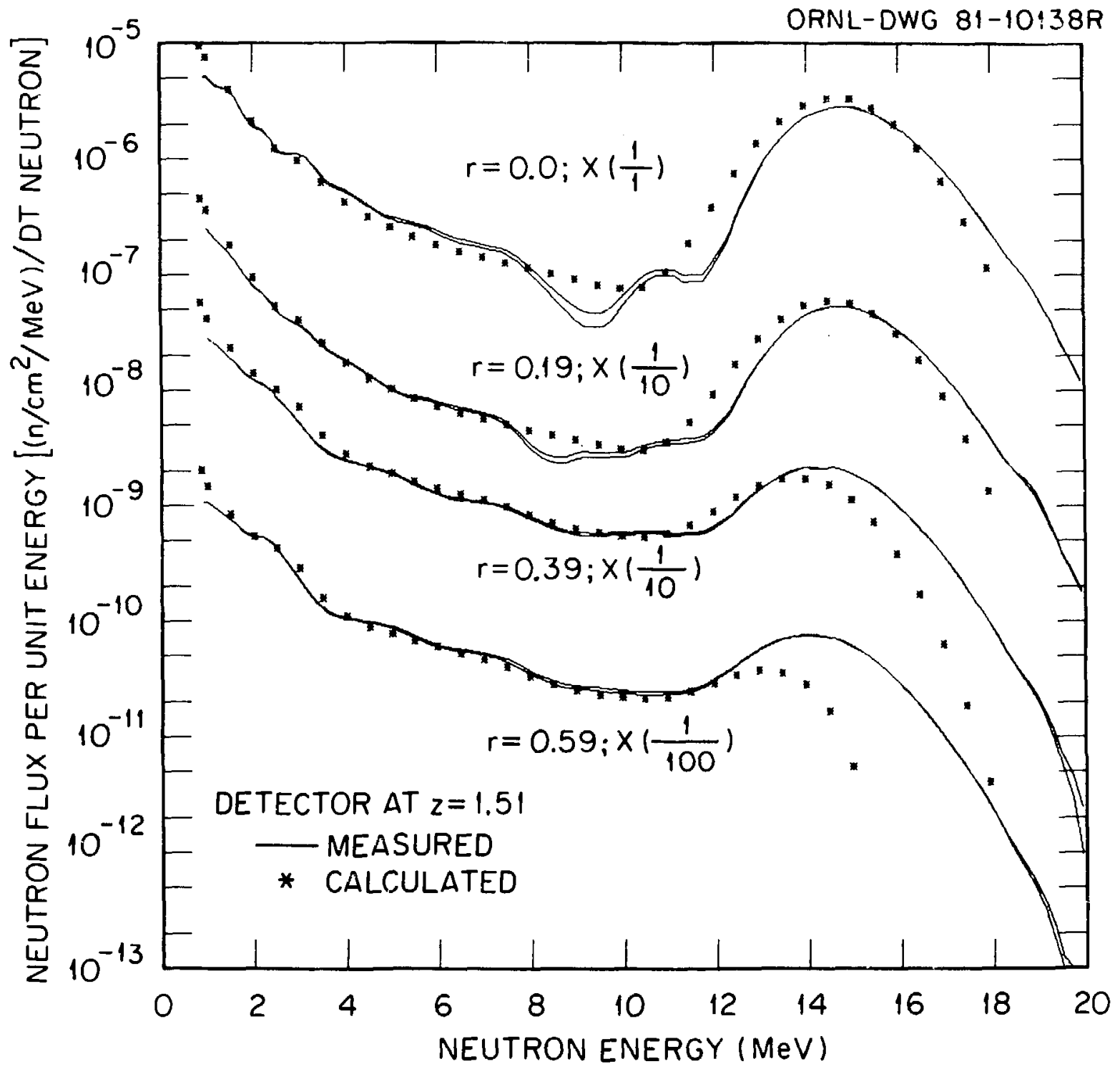

Fig. 6. Neutron flux per unit energy versus neutron energy for the detectors at $\mathrm{z}=1.51 \mathrm{~m}$; $r=0.0,0.19,0.39$, and $0.59 \mathrm{~m}$. The calculated data were obtained using discrete ordinates radiation transport methods. 


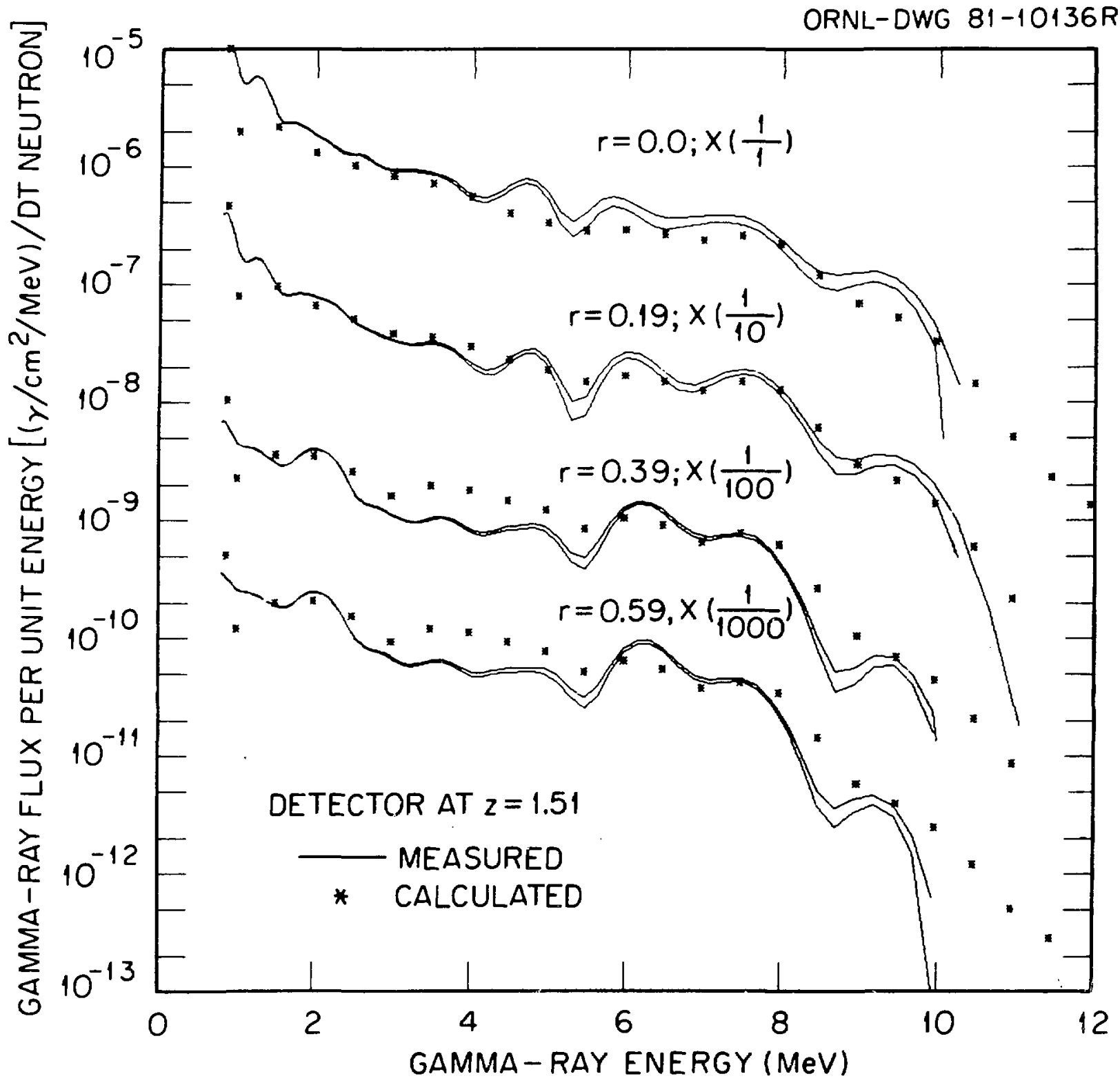

Fig. 7. Gamma-ray flux per unit energy versus gamma-ray energy for the detectors at $z=$ $1.51 \mathrm{~m} ; \mathrm{r}=0.0,0.19,0.39$, and $0.59 \mathrm{~m}$. The calculated data were obtained using discrete ordinates radiation transport methods. 
detail in Ref. 5 so only a brief discussion will be given here. Basically, for neutron energies $>10 \mathrm{MeV}$, the first collision contribution to the neutron flux per unit energy as obtained by discrete ordinates methods using the $\mathrm{P}_{3}$ Legendre expansion is removed, i.e., subtracted, from the total neutron flux per unit energy and a Monte Carlo estimate of this first collision contribution is used. The Monte Carlo estimate is obtained using a Monte Carlo code that samples directly from the angular distributions that are included in the ENDF/B-IV file. In the work reported here, the recently developed Monte Carlo code PX MORSE ${ }^{12}$ was used to estimate the single scattering contribution, but any other continuous energy-angle Monte Carlo code could be used.

In Fig. 8 the calculated and experimental neutron flux per unit energy are compared for the same detector locations that were considered in Fig. 6; i.e., the experimental data in Fig. 8 are the same as those in Fig. 6.

As in Fig. 6, the solid curves show the measured spectra and the points are the calculated data. In Fig. 8, two sets of calculated points are given "for some detector locations at neutron energies above $10 \mathrm{MeV}$. These points indcate the upper and lower bounds on the calculated spectra due to the statistical variation in the estimated single scattered neutron scalar flux introduced by the Monte Carlo calculations. The band defined by these points represents plus or minus one standard deviation in the calculated data.

Comparison of these data with those shown in Fig. 6 reveal that significant improvement at the higher energies has been achieved among the calculated and experimental spectra when the calculated data are obtained using the improved treatment of neutron single scattering.

Consider the cases where the detector is on the axis of the duct, i.e., $r=0.0$. The agreement between the calculated and measured neutron spectra is favorable above $\sim 10 \mathrm{MeV}$. In the neutron energy range between $\sim_{11}$ and $\sim_{13} \mathrm{MeV}$, the calculated data are higher than the measured data, but the difference between the calculated and measured data is reduced compared to the same spectra shown in Fig. 6 . 


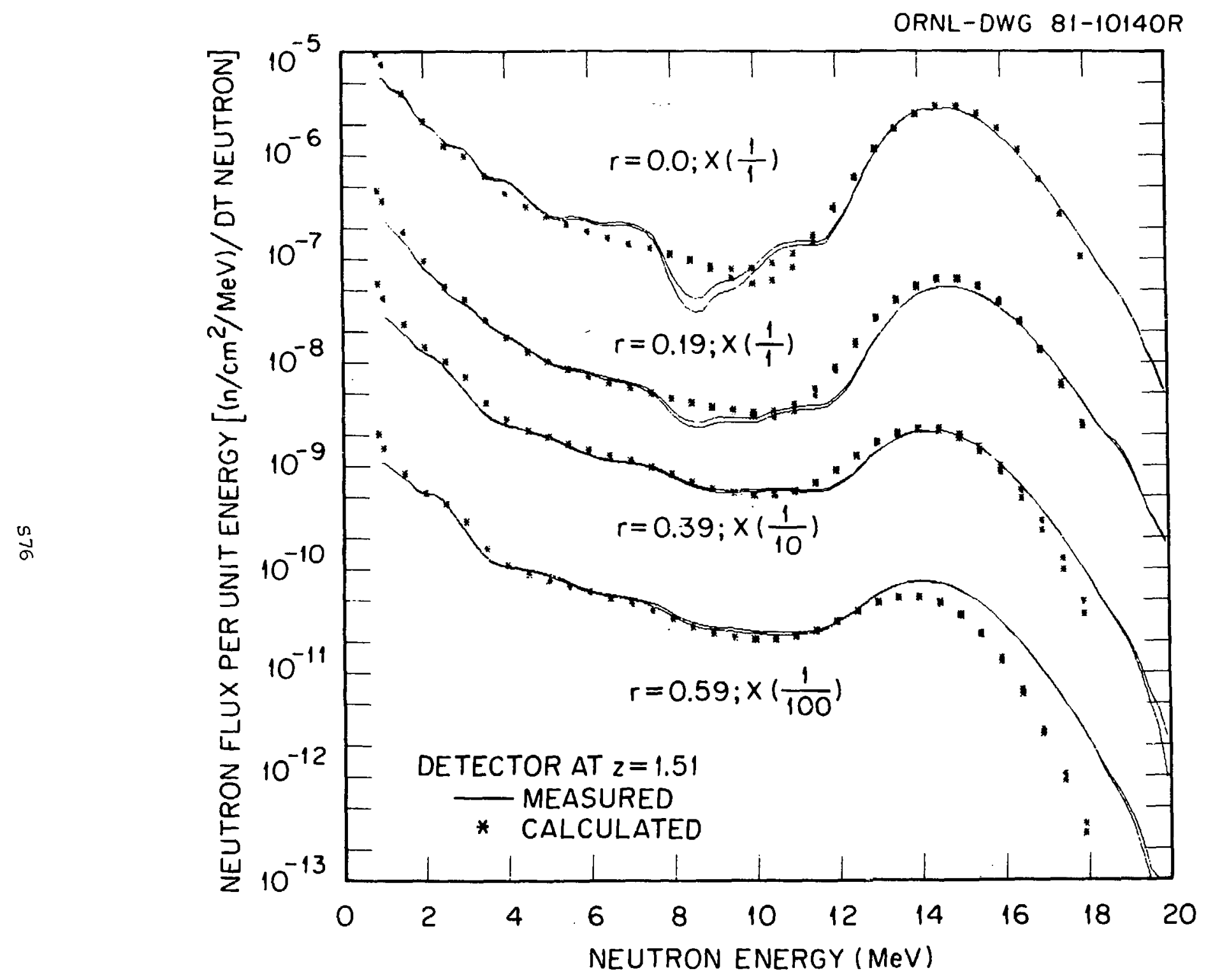

Fig. 8. Neutron flux per unit energy versus neutron energy for the detectors at $2=1.51 \mathrm{~m}$; $\mathbf{r}=0.0,0.19,0.39$, and $0.59 \mathrm{~m}$. The calculated data were obtained using a combination of discrete ordinates and Monte Carlo radiation transport methods. 


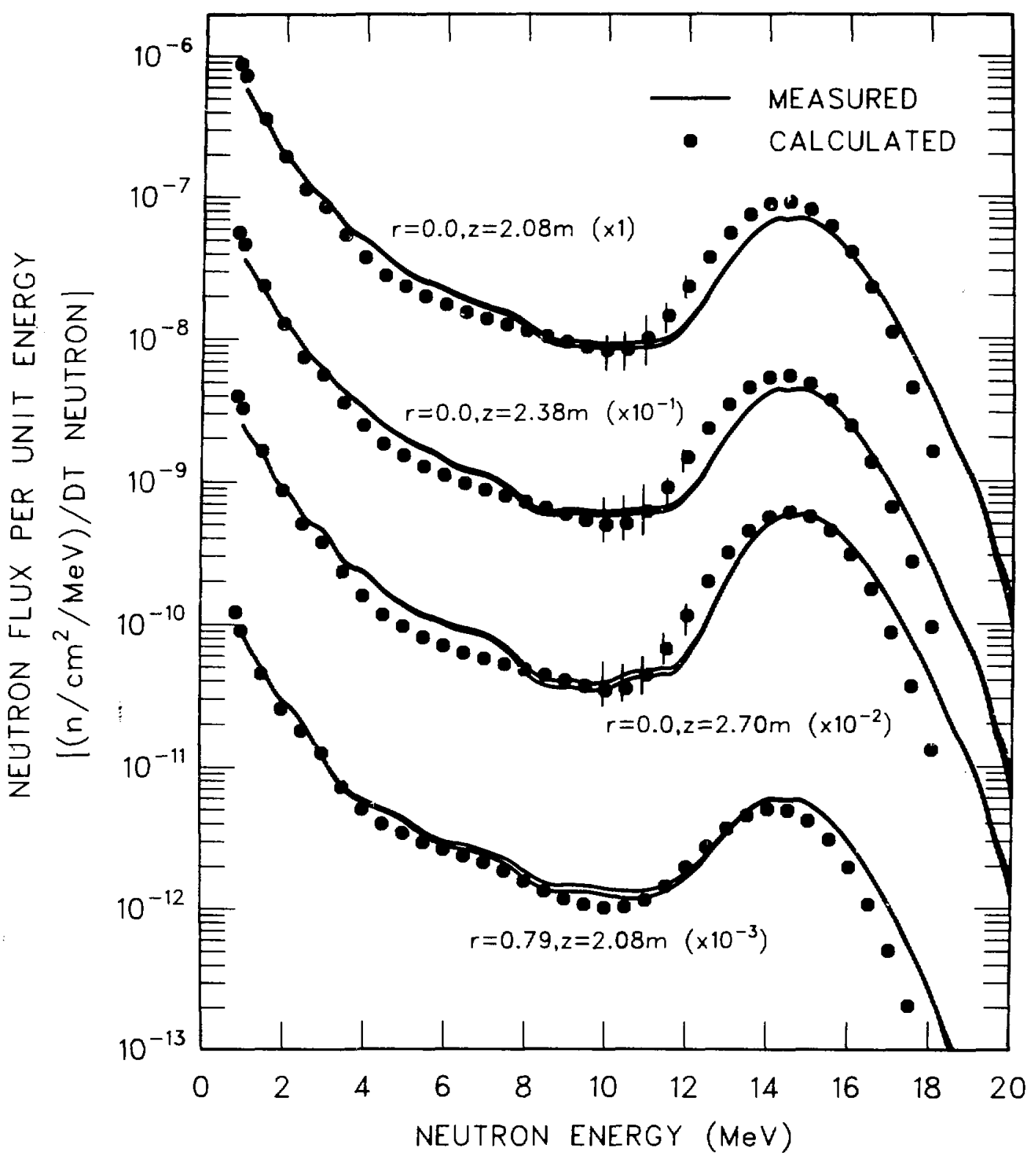

Fig. 9. Neutron flux per unit energy versus neutron energy versus neutron energy for the detectors at $r=0.0 \mathrm{~m}$, and $\mathrm{z}=2.08,2.38$, and $2.70 \mathrm{~m}$ and at $\mathrm{r}=0.79 \mathrm{~m}, \mathrm{z}=2.08 \mathrm{~m}$. The results in this figure are for the case when the shadow bar (see Fig. 5) is in place. The calculations were carried out using a combination of discrete ordinates and Monte Carlo transport methods. 


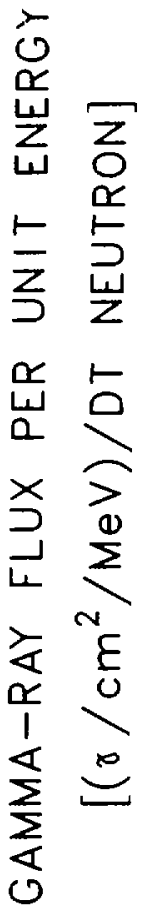

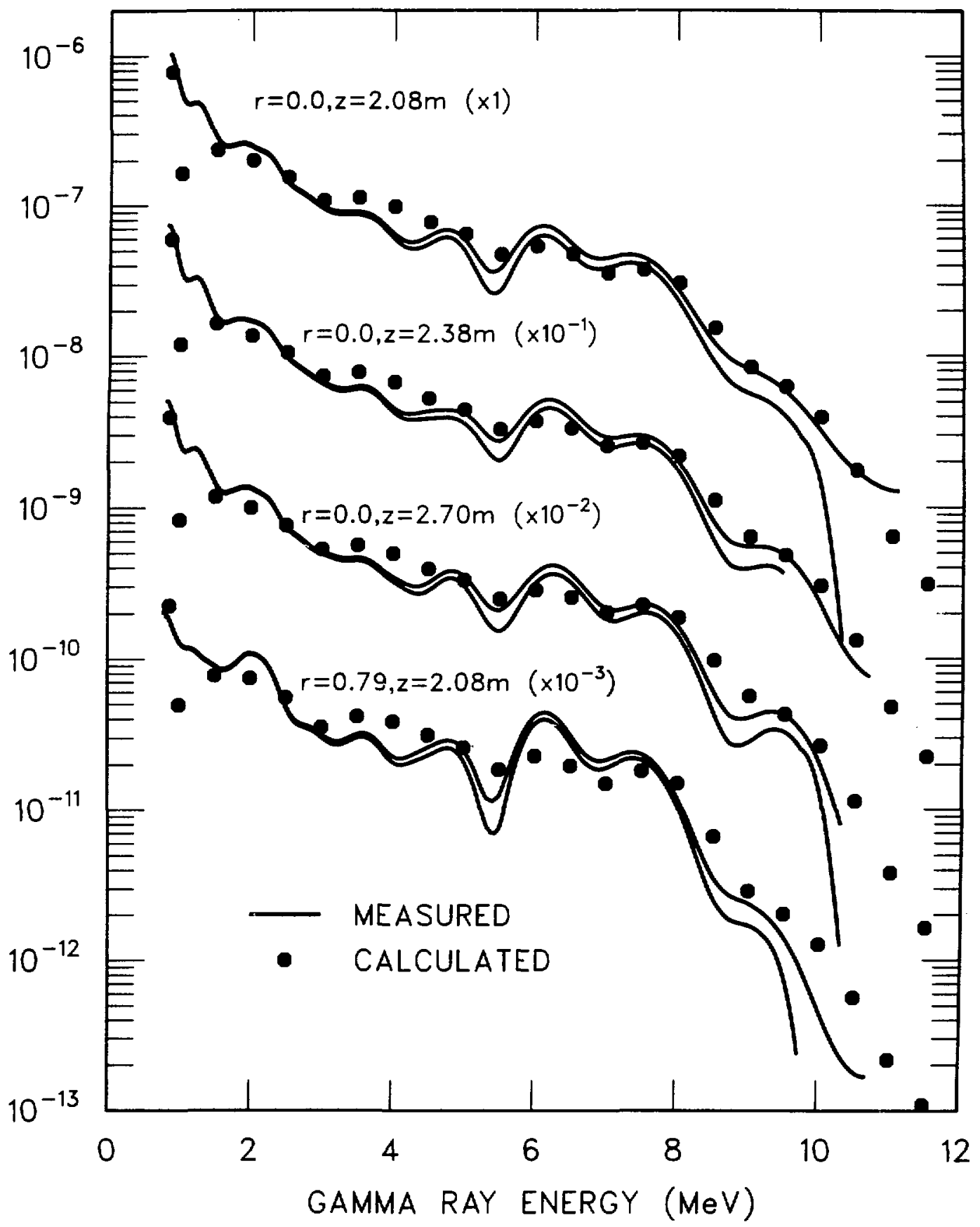

Fig. 10. Gamma-ray flux per unit energy versus gamma-ray energy for the detectors at $\mathbf{r}=$ $0.0 \mathrm{~m}$ and $\mathrm{z}=2.08,2.38$, and $2.70 \mathrm{~m}$ and $\mathrm{r}=0.79 \mathrm{~m}$ and $\mathrm{z}=2.08 \mathrm{~m}$. The calculations were carried out using discrete ordinates radiation transport methods. 
Calculated results obtained are compared with the data measured when the shadow bar was in place (see Fig. 5) in Figs. 9 and 10. The calculated neutron results were obtained using a combination of discrete ordinates and Monte Carlo methods as explained above and the calculated gamma-ray results were obtained using only discrete ordinates methods. The degree of agreement between the calculated and measured neutron spectra in Fig. 9 is similar to that shown in Fig. 8. Similarly, the degree of agreement between the calculated and experimental gamma ray data in Fig. 10 is comparable to that shown in Fig. 7 .

\section{Monte Carlo Transport Methods}

In this subsection, calculated results obtained using entirely Monte Carlo methods are presented and combared witn the experimental data. The geometry used in the calculations is that shown in Fig. 4. Calculational results obtained using Monte Carlo methods for the configuration in Fig. 5, i.e., the configuration with the shadow bar in place, have not been made.

The calculations were carried out with the code MCNP.13 MCNP is a general purpose, continous-energy, generalized geometry, time-dependent coupled neutron-photon Monte Carlo transport code. Pointwise cross section data are treated in considerable detall in energy grids that are tailored for each isotope in the cross section data libraries. The angular distributions for elastic and inelastic scattering are also described on a fine grid of incident neutron energies and linear interpolation methods are used to obtain the angular distribution of the scattered neutrons vs. incoming neutron energy. The angular distributions of the outgoing particles are then sampled in a continuous fashion. In these calculations, however, the reflection coefficient of 0.2 for neutrons and gamma rays at the radial boundary of the two-dimensional configuration (see Fig. 2) was not included; i.e., particles were allowed to escape from these boundaries without reflection. Much more detail concerning the code will be found in Ref. 13.

The energy-angle distribution of the source neutrons was taken into account in the same manner as that described previously. The calculations 
were carried out using cross section data based on ENDF/B-V and are those available at the National Magnetic Fusion Energy Computing Center for use with MCNP. More details concerning the calculational procedure will be found in Ref. 7 .

The Monte Carlo fluxes were binned into energy intervals having the same widths as those used in the calculations in Refs. 1, 5, 6, and 7 and the smoothing of the MCNP results with the detector response was carried out in the same manner as in these references. The error bars on the MCNP results correspond to one standard deviation. When no error bars are shown, they are smaller than the plotted points. The error bars were obtained by separately smoothing the upper and lower bounds of the fluxes determined from the statistical variation in the fluxes in each energy bin.

In Fig. 11 the calculated and experimental neutron fluxes per unit energy per source neutron for detector locations 8, 9, and 11 in Fig. 4 are shown. The corresponding gamma-ray fluxes at detectors 8 and 9 are shown in Fig. 12.

Examination of Figs. 11 and 12 shows that the spectra calculated with MCNP are generally in good agreement with the experimental data. 
ORNL-DWG $82 \mathrm{C}-15095$

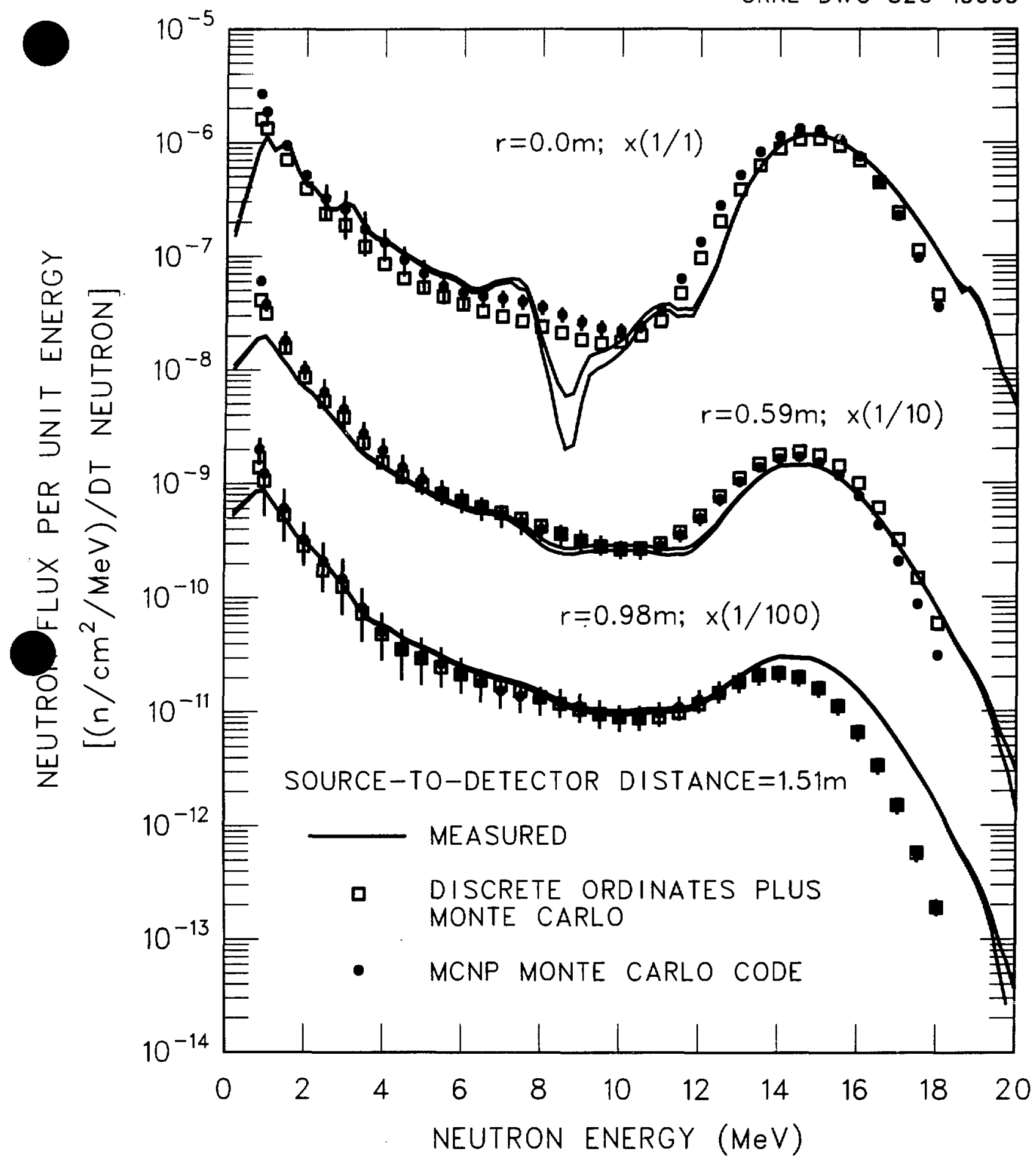

Fig. 11 Neutron flux per unit energy versus neutron energy for a source-to-detector distance of $1.51 \mathrm{~m}$ and radial distances of $0.0,0.59$, and $0.98 \mathrm{~m}$ from the axis. The target is at $\mathrm{z}$ $=0.57$ so the $z$ position of the detector in the coordinate system of Fig. 4 is $2.08 \mathrm{~m}$. 


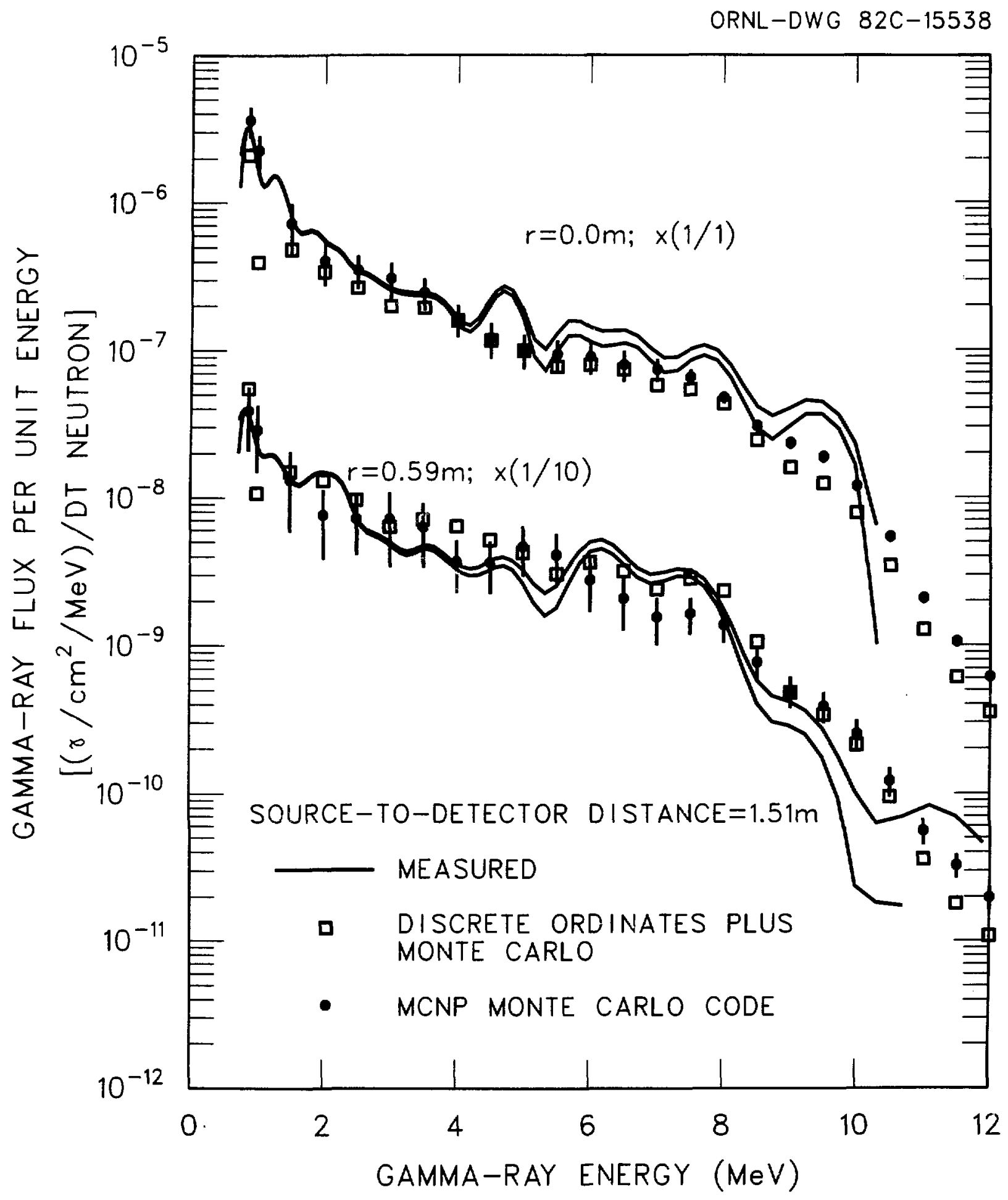

Fig. 12. Gamma-ray hux per unit energy versus gamma-ray energy for a source-to-detector distance of $1.51 \mathrm{~m}$ and radial distances of 0.0 and $0.59 \mathrm{~m}$ from the axis. The target is at $\mathrm{z}=$ $0.57 \mathrm{~m}$ so the $\mathrm{z}$ position of the detector in the coordinate svstem of Fig. 4 is $2.08 \mathrm{~m}$. 
1. Cross Section Evaluation Working Group Benchmark Specifications, Benchmark SB5, "Fusion Reactor Shielding Benchmark", BNL-19302, Vo1. II, Brookhaven National Laboratory (1983).

2. R. T. Santoro et al., "Calculational Procedures for the Analysis of Integral Experiments for Fusion Reactor Design", ORNL/TM-5777, Oak Ridge National Laboratory (1981).

3. T. R. Fewe11, "Absolute Determination of 14-MeV Neutron Yields", SC-RR67-346, Sandia Laboratories (1967).

4. W. R. Burrus and V.V. Verbinski, "Fast-Neutron Spectroscopy With Thick Organic Scintillators", Nucl. Inst. Methods 67, 181 (1969).

5. R. T. Santoro et al., "Calculated Neutron and Gamma-Ray Energy Spectra From 14-MeV Neutrons Streaming Through an Iron Duct: Comparison with Experiment", Nucl. Sci. Eng. 80, 586 (1982).

6. R. T. Santoro et al., "Comparison of Measured and Calculated Neutron and Gamma-Ray Energy Spectra, Behind an In-Line Shielded Duct", J. of Fusion Energy $\underline{2}, 6$ (1982).

7. R. T. Santoro et al., "Streaming of 14-MeV Neutrons Through an Iron Duct - Comparison of Measured Neutron and Gamma-Ray Energy Spectra with Results Calculated Using the Monte Carlo MCNP Code", Nucl. Sci. Eng. 14 , 260 (1983).

8. ORNL/RSIC-37 Radiation Shielding Information Center, Oak Ridge National Laboratory (1975), available as DLC/41-VITAMIN C. 


\section{REFERENCES (cont'd)}

9. W. A. Rhoades and F. R. Mynatt, "The DOT III Two-Dimensional Discrete Ordinates Radiation Transport Code", ORNL/TM-4280,. Oak Ridge National Laboratory (1979).

10. R. A. Lillie, R. G. Alsmiller, Jr., and J. T. Mihalczo, "Design Calculations for a $14-\mathrm{MeV}$ Neutron Collimator", Nuc1. Techno1. 43, 373 (1979).

11. "FALSTF, Informa1 Notes", CCC-351, Radiation Shielding Information Center, Oak Ridge National Laboratory (1979).

12. M. M. Wadel1, Jr., "PXMORSE, A Continuous Energy Monte Carlo Code", Ph.D. Thesis, University of Tennessee (1981).

13. "MCNP - A General Purpose Monte Carlo Code for Neutron and Photon Transport", LA-7396-M (Rev.) Los Alamos National Laboratory, Los Alamos Monte Carlo Group (1981). 


\section{Appendix A: Tabulation of Unfolded Experimental Data .}

The tabulations on the following pages show the numerical values of the final unfolded data for both neutrons and gamma rays. The energy is given in $\mathrm{MeV}$ and the upper and lower values of the confidence band \pm one standard deviation are given in neutrons or gamma rays per $\mathrm{cm}^{2}$ per MeV per source neutron. The negative values occurring at the higher energies result from supplying bins to the unfolding program that contain no counts. The program "expects" data for the total light unit range and returns data for this range. These negative data points are not significant and are included here only to show the complete calculated data set.

In the experiment, the detector position was specified in a right-handed coordinate system with the z-axis corresponding to the deuteron beam axis and the x-axis parallel to the experimental room floor. The off-axis detector locations were positioned on the positive $x$ axis. The detector coordinates $x$, $y, z^{\prime}$ are specified in each of the tables. The quantity $z^{\prime}$ is the source-todetector distance. Since the target is located at $z=57.0 \mathrm{~cm}$, the $z$ position of the detector in the coordinate system of Figs. 4 and 5 is obtained by adding $57.0 \mathrm{~cm}$ to the $z^{\prime}$ value given in the following tables. For a given detector location, the neutron data are presented and then the gamma-ray data. The detector positions are considered in the order they are listed in Table 1 . 
Table A. 1

Neutron Data

Detector 1 in Fig. 4

Detector Coordinates $(\mathrm{cm}): x=0.0, y=0.0, z^{\prime}=94.0$

\begin{tabular}{|c|c|c|c|c|c|}
\hline 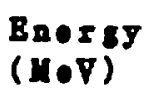 & 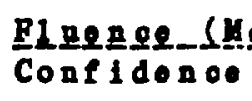 & 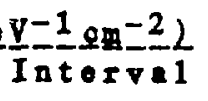 & $\begin{array}{l}B n \bullet \mathcal{E g} \\
(\mu \circ V)\end{array}$ & 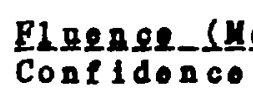 & $\frac{y-1}{\text { Inter }}{ }^{-1}-\frac{2}{2} L$ \\
\hline $\begin{array}{l}0.91 \\
1.01 \\
1.11 \\
1.21 \\
1.31 \\
1.41 \\
1.51 \\
1.61 \\
1.71 \\
1.81 \\
1.94 \\
2.11 \\
2.31 \\
2.50 \\
2.69 \\
2.90 \\
3.10 \\
3.30 \\
3.51 \\
3.71 \\
3.90 \\
4.15 \\
4.46 \\
4.75 \\
5.06 \\
5.36 \\
5.64\end{array}$ & $\begin{array}{l}5.32 \mathrm{E}-06 \\
5.13 \mathrm{E}-06 \\
4.65 \mathrm{E}-06 \\
4.27 \mathrm{E}-06 \\
4.12 \mathrm{E}-06 \\
4.03 \mathrm{E}-06 \\
3.82 \mathrm{E}-06 \\
3.42 \mathrm{E}-06 \\
2.89 \mathrm{E}-06 \\
2.37 \mathrm{E}-06 \\
1.95 \mathrm{E}-06 \\
1.82 \mathrm{E}-06 \\
1.67 \mathrm{E}-06 \\
1.3: 0 \mathrm{E}-06 \\
1.07 \mathrm{E}-06 \\
1.11 \mathrm{E}-06 \\
1.11 \mathrm{E}-06 \\
9.35 \mathrm{E}-07 \\
7.02 \mathrm{E}-07 \\
5.80 \mathrm{E}-07 \\
5.47 \mathrm{E}-07 \\
4.95 \mathrm{E}-07 \\
4.06 \mathrm{E}-07 \\
3.38 \mathrm{E}-07 \\
2.96 \mathrm{~B}-07 \\
2.73 \mathrm{E}-07 \\
2.61 \mathrm{E}-07\end{array}$ & $\begin{array}{l}5.37 \mathrm{E}-06 \\
5.17 \mathrm{E}-06 \\
4.69 \mathrm{E}-06 \\
4.31 \mathrm{E}-06 \\
4.16 \mathrm{E}-06 \\
4.07 \mathrm{E}-06 \\
3.86 \mathrm{E}-06 \\
3.45 \mathrm{E}-06 \\
2.91 \mathrm{E}-06 \\
2.40 \mathrm{E}-06 \\
1.99 \mathrm{E}-06 \\
1.84 \mathrm{E}-06 \\
1.69 \mathrm{E}-06 \\
1.32 \mathrm{E}-06 \\
1.09 \mathrm{E}-06 \\
1.13 \mathrm{E}-06 \\
1.14 \mathrm{E}-06 \\
9.52 \mathrm{E}-07 \\
7.22 \mathrm{E}-07 \\
6.03 \mathrm{E}-07 \\
5.62 \mathrm{E}-07 \\
5.12 \mathrm{E}-07 \\
4.17 \mathrm{E}-07 \\
3.50 \mathrm{E}-07 \\
3.10 \mathrm{E}-07 \\
2.89 \mathrm{E}-07 \\
2.74 \mathrm{E}-07\end{array}$ & $\begin{array}{l}5.94 \\
6.25 \\
6.55 \\
6.83 \\
7.23 \\
7.73 \\
8.25 \\
8.77 \\
9.24 \\
9.72 \\
10.23 \\
10.75 \\
11.26 \\
11.76 \\
12.40 \\
13.20 \\
14.03 \\
14.86 \\
15.62 \\
16.45 \\
17.44 \\
18.51 \\
19.58 \\
20.33 \\
21.43 \\
22.40 \\
23.43\end{array}$ & $\begin{array}{r}2.39 \mathrm{E}-07 \\
2.05 \mathrm{E}-07 \\
1.85 \mathrm{E}-07 \\
1.76 \mathrm{E}-07 \\
1.62 \mathrm{E}-07 \\
1.36 \mathrm{E}-07 \\
8.58 \mathrm{E}-08 \\
5.21 \mathrm{E}-08 \\
3.52 \mathrm{E}-08 \\
3.55 \mathrm{E}-08 \\
6.45 \mathrm{E}-08 \\
9.66 \mathrm{E}-08 \\
9.67 \mathrm{E}-08 \\
8.25 \mathrm{E}-08 \\
2.18 \mathrm{E}-07 \\
1.00 \mathrm{E}-06 \\
2.26 \mathrm{E}-06 \\
2.84 \mathrm{E}-06 \\
2.37 \mathrm{E}-06 \\
1.39 \mathrm{E}-06 \\
5.25 \mathrm{E}-07 \\
1.32 \mathrm{E}-07 \\
2.45 \mathrm{E}-08 \\
1.27 \mathrm{E}-09 \\
-4.92 \mathrm{~B}-09 \\
-6.66 \mathrm{~B}-09 \\
-6.01 \mathrm{E}-09\end{array}$ & $\begin{array}{r}2.51 \mathrm{E}-07 \\
2.19 \mathrm{E}-07 \\
2.00 \mathrm{E}-07 \\
1.89 \mathrm{E}-07 \\
1.74 \mathrm{E}-07 \\
1.50 \mathrm{E}-07 \\
9.86 \mathrm{E}-08 \\
6.35 \mathrm{E}-08 \\
4.75 \mathrm{E}-08 \\
4.69 \mathrm{E}-08 \\
7.65 \mathrm{E}-08 \\
1.10 \mathrm{E}-07 \\
1.10 \mathrm{E}-07 \\
9.53 \mathrm{E}-08 \\
2.31 \mathrm{E}-07 \\
1.01 \mathrm{E}-06 \\
2.27 \mathrm{E}-06 \\
2.84 \mathrm{E}-06 \\
2.38 \mathrm{E}-06 \\
1.39 \mathrm{E}-06 \\
5.27 \mathrm{E}-07 \\
1.33 \mathrm{E}-07 \\
2.49 \mathrm{E}-08 \\
1.47 \mathrm{E}-09 \\
-4.79 \mathrm{E}-09 \\
-6.52 \mathrm{E}-09 \\
-5.88 \mathrm{E}-09\end{array}$ \\
\hline
\end{tabular}


Table A.1 (Cont'd)

Gamma-Ray Data

Detector 1 in Fig. 4

Detector Coordinates $(\mathrm{cm}): \quad x=0.0, y=0.0, z^{\prime}=94.0$

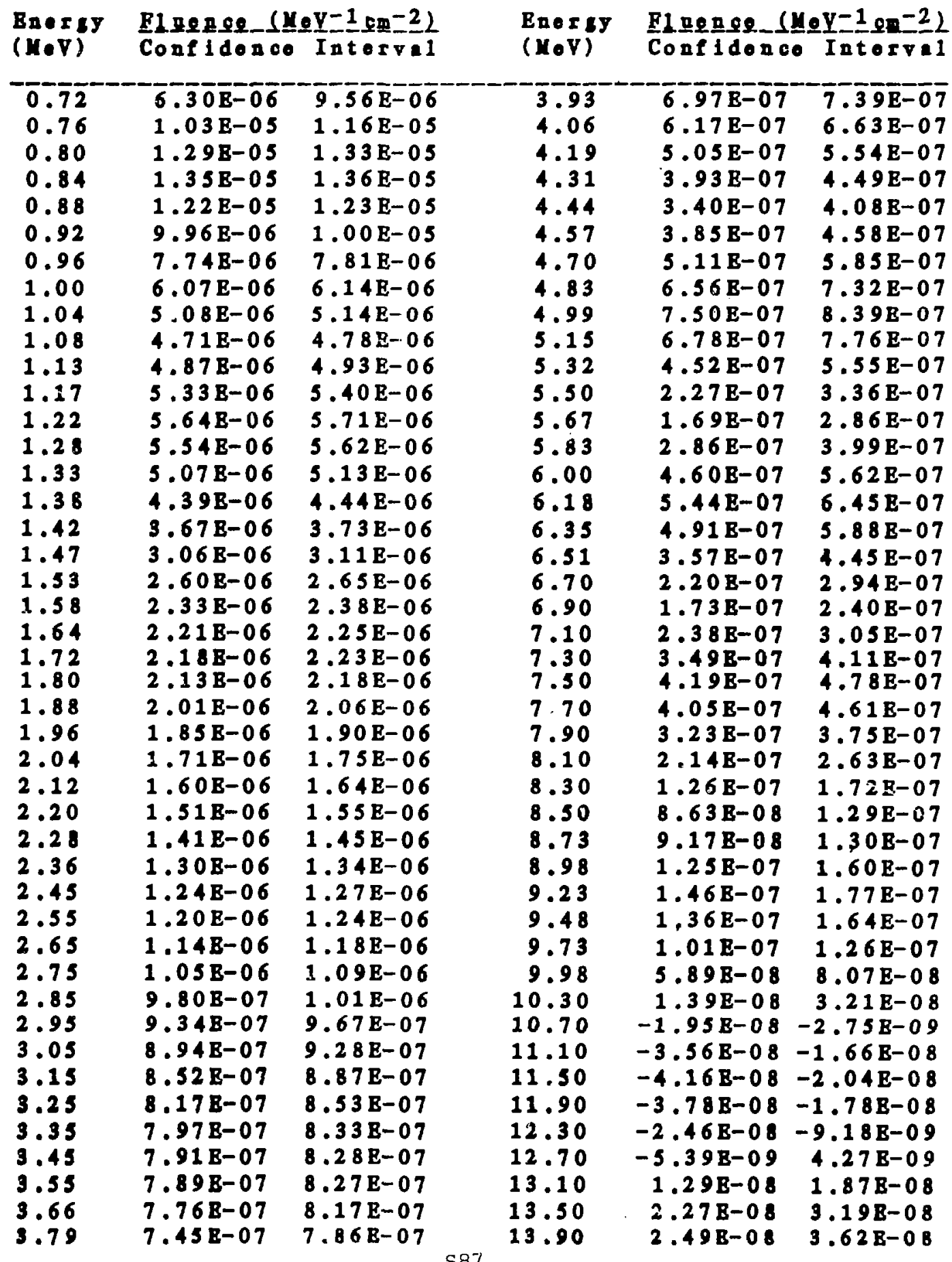


Table A.2

Neutron Data

Detector 2 in Fig. 4

Detector Coordinates $(\mathrm{cm}): x=19.0, y=0.0, z^{\prime}=94.0$

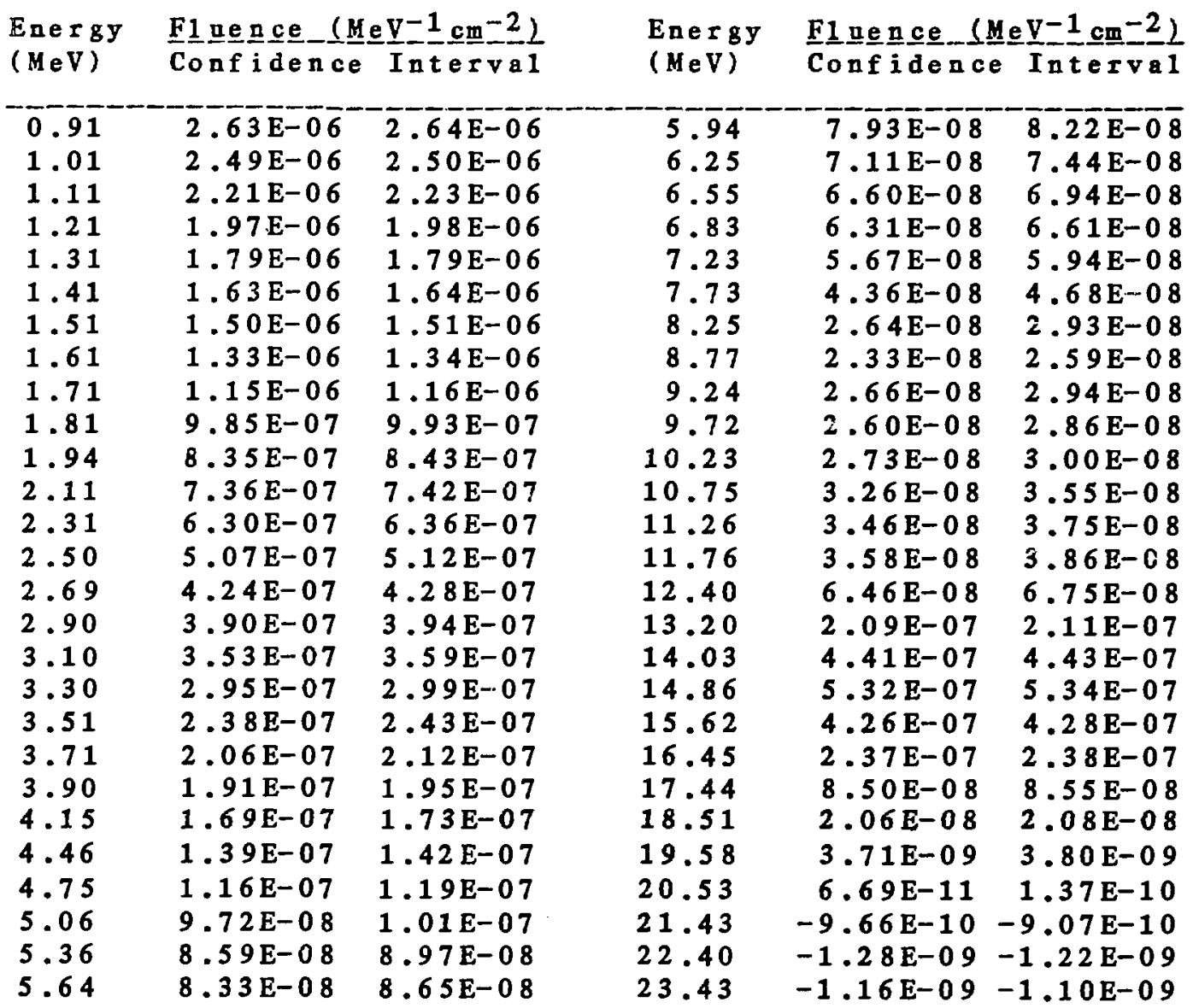


Table A.2 (Cont'd)

Gamma-Ray Data

Detector 2 in Fig. 4

Detector Coordinates $(\mathrm{cm}): x=19.0, y=0.0, z^{\prime}=94.0$

( $\mathrm{HeV}$ ) Confidence Interval

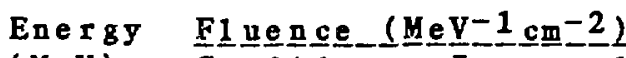

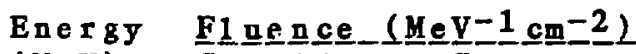
(MeV) Confidence Interval

\begin{tabular}{|c|c|c|c|c|c|}
\hline 0.72 & $2.18 \mathrm{E}-06$ & $3.21 \mathrm{E}-06$ & 3.93 & $2.28 \mathrm{E}-07$ & $2.41 \mathrm{E}-07$ \\
\hline 0.76 & $3.28 \mathrm{E}-06$ & $3.70 \mathrm{E}-06$ & 4.06 & $1.92 \mathrm{E}-07$ & $2.07 \mathrm{E}-07$ \\
\hline 0.80 & $3.98 \mathrm{E}-06$ & $4.10 \mathrm{E}-06$ & 4.19 & $1.73 \mathrm{E}-07$ & 1. $88 \mathrm{E}-07$ \\
\hline 0.84 & $4.08 \mathrm{E}-06$ & $4.12 \mathrm{E}-06$ & 4.31 & 1. $75 \mathrm{E}-07$ & $1.93 \mathrm{E}-07$ \\
\hline 0.88 & $3.68 \mathrm{E}-06$ & $3.71 \mathrm{E}-06$ & 4.44 & $1.98 \mathrm{E}-07$ & $2.19 \mathrm{E}-07$ \\
\hline 0.92 & $3.04 \mathrm{E}-06$ & $3.07 \mathrm{E}-06$ & 4.57 & $2.33 \mathrm{E}-07$ & $2.55 \mathrm{E}-07$ \\
\hline 0.96 & $2.41 \mathrm{E}-06$ & $2.44 \mathrm{E}-06$ & 4.70 & $2.62 \mathrm{E}-07$ & $2.85 \mathrm{E}-07$ \\
\hline 1.00 & $1.94 \mathrm{E}-06$ & $1.97 \mathrm{E}-06$ & 4.83 & $2.62 \mathrm{E}-07$ & $2.86 \mathrm{E}-07$ \\
\hline 1.04 & $1.66 \mathrm{E}-06$ & $1.68 \mathrm{E}-06$ & 4.99 & $2.13 \mathrm{E}-07$ & $2.42 \mathrm{E}-07$ \\
\hline 1.08 & $1.55 \mathrm{E}-06$ & $1.57 \mathrm{E}-06$ & 5.15 & $1.27 \mathrm{E}-07$ & $1.58 \mathrm{E}-07$ \\
\hline 1.13 & $1.55 \mathrm{E}-06$ & $1.57 \mathrm{E}-06$ & 5.32 & $7.10 \mathrm{E}-08$ & $1.02 \mathrm{E}-07$ \\
\hline 1.17 & $1.62 \mathrm{E}-06$ & $1.64 \mathrm{E}-06$ & 5.50 & $7.80 \mathrm{E}-08$ & $1.12 \mathrm{E}-07$ \\
\hline 1.22 & $1.66 \mathrm{E}-06$ & $1.69 \mathrm{E}-06$ & 5.67 & $1.37 \mathrm{E}-07$ & $1.73 \mathrm{E}-07$ \\
\hline 1.28 & $1.63 \mathrm{E}-06$ & $1.66 \mathrm{E}-06$ & 5.83 & $2.03 \mathrm{E}-07$ & $2.37 \mathrm{E}-07$ \\
\hline 1.33 & $1.50 \mathrm{E}-06$ & $1.53 \mathrm{E}-06$ & 6.00 & $2.38 \mathrm{E}-07$ & $2.68 \mathrm{E}-07$ \\
\hline 1.38 & $1.32 \mathrm{E}-06$ & $1.34 \mathrm{E}-06$ & 6.18 & $2.30 \mathrm{E}-07$ & $2.62 \mathrm{E}-07$ \\
\hline 1.42 & $1.14 \mathrm{E}-06$ & $1.16 \mathrm{E}-06$ & 6.35 & $1.94 \mathrm{E}-07$ & $2.24 E-07$ \\
\hline 1.47 & $9.87 \mathrm{E}-07$ & $1.00 \mathrm{E}-06$ & 6.51 & $1.54 E-07$ & $1.81 \mathrm{E}-07$ \\
\hline 1.53 & $8.77 \mathrm{E}-07$ & $8.91 \mathrm{E}-07$ & 6.70 & $1.26 \mathrm{E}-07$ & $1.48 \mathrm{E}-07$ \\
\hline 1.58 & $8.16 \mathrm{E}-07$ & $8.31 \mathrm{E}-07$ & 6.90 & $1.22 \mathrm{E}-07$ & $1.42 \mathrm{E}-07$ \\
\hline $\begin{array}{l}1.64 \\
1.72 \\
1.80\end{array}$ & $\begin{array}{l}8.00 \mathrm{E}-07 \\
8.20 \mathrm{E}-07 \\
8.29 \mathrm{E}-07\end{array}$ & $\begin{array}{l}8.14 \mathrm{E}-07 \\
8.33 \mathrm{E}-07 \\
8.42 \mathrm{E}-07\end{array}$ & & $\begin{array}{l}1.40 \mathrm{E}-07 \\
1.63 \mathrm{E}-07 \\
1.74 \mathrm{E}-07\end{array}$ & $\begin{array}{l}1.60 \mathrm{E}-07 \\
1.81 \mathrm{E}-07 \\
1.91 \mathrm{E}-07\end{array}$ \\
\hline 1.88 & $8.20 \mathrm{E}-07$ & $8.33 \mathrm{E}-07$ & 7.70 & $1.64 \mathrm{E}-07$ & $1.80 \mathrm{E}-07$ \\
\hline 1.96 & $7.94 \mathrm{E}-07$ & $8.09 \mathrm{E}-07$ & 7.90 & $1.36 \mathrm{E}-07$ & $1.51 \mathrm{E}-07$ \\
\hline 2.04 & $7.60 \mathrm{E}-07$ & $7.75 \mathrm{E}-07$ & 8.10 & $9.81 \mathrm{E}-08$ & $1.11 \mathrm{E}-07$ \\
\hline 2.12 & $7.30 \mathrm{E}-07$ & $7.45 \mathrm{E}-07$ & 8.30 & $6.25 \mathrm{E}-08$ & $7.42 \mathrm{E}-08$ \\
\hline 2.20 & $6.99 \mathrm{E}-07$ & $7.13 \mathrm{E}-07$ & 8.50 & $3.79 \mathrm{E}-08$ & $4.83 \mathrm{E}-08$ \\
\hline 2.28 & $6.46 \mathrm{E}-07$ & $6.59 \mathrm{E}-07$ & 8.73 & $2.53 \mathrm{E}-08$ & $3.48 \mathrm{E}-08$ \\
\hline 2.36 & $5.73 E-07$ & $5.85 \mathrm{E}-07$ & 8.98 & $2.49 \mathrm{E}-08$ & $3.34 \mathrm{E}-08$ \\
\hline 2.45 & $5.00 \mathrm{E}-07$ & $5.11 \cdot E-07$ & 9.23 & $2.90 E-08$ & $3.63 \mathrm{E}-08$ \\
\hline 2.55 & $4.56 \mathrm{E}-07$ & $4.66 \mathrm{E}-07$ & 9.48 & $2.95 \mathrm{E}-08$ & $3.59 \mathrm{E}-08$ \\
\hline 2.65 & $4.29 \mathrm{E}-07$ & $4.40 \mathrm{E}-07$ & 9.73 & $2.45 \mathrm{E}-08$ & $3.00 \mathrm{E}-08$ \\
\hline 2.75 & $3.97 \mathrm{E}-07$ & $4.08 \mathrm{E}-07$ & 9.98 & $1.60 \mathrm{E}-08$ & $2.08 \mathrm{E}-08$ \\
\hline 2.85 & $3.65 \mathrm{E}-07$ & $3.76 \mathrm{E}-07$ & 10.30 & $4.90 \mathrm{E}-09$ & $9.44 \mathrm{E}-09$ \\
\hline 2.95 & $3.44 \mathrm{E}-07$ & $3.55 \mathrm{E}-07$ & 10.70 & $-3.09 E-09$ & $1.74 E-09$ \\
\hline 3.05 & $3.28 \mathrm{E}-07$ & $3.39 \mathrm{E}-07$ & 11.10 & $-5.08 \mathrm{E}-09$ & $1.77 \mathrm{E}-10$ \\
\hline 3.15 & $3.15 \mathrm{E}-07$ & $3.26 \mathrm{E}-07$ & 11.50 & $-4.17 E-09$ & $1.05 \mathrm{E}-09$ \\
\hline 3.25 & $3.07 \mathrm{E}-07$ & $3.19 \mathrm{E}-07$ & 11.90 & $-2.26 \mathrm{E}-09$ & $2.30 \mathrm{E}-09$ \\
\hline 3.35 & $3.10 \mathrm{E}-07$ & $3.21 \mathrm{E}-07$ & 12.30 & $-2.05 E-10$ & $3.24 \mathrm{E}-09$ \\
\hline 3.45 & $3.17 \mathrm{E}-07$ & $3.29 \mathrm{E}-07$ & 12.70 & $1.59 \mathrm{E}-09$ & $3.72 \mathrm{E}-09$ \\
\hline 3.55 & $3.19 \mathrm{E}-07$ & $3.31 \mathrm{E}-07$ & 13.10 & $2.34 \mathrm{E}-09$ & $4.23 \mathrm{E}-09$ \\
\hline 3.66 & $3.05 \mathrm{E}-07$ & $3.18 \mathrm{E}-07$ & 13.50 & $1.65 \mathrm{E}-09$ & $4.43 \mathrm{E}-09$ \\
\hline 3.79 & $2.70 \mathrm{E}-07$ & $2.83 \mathrm{E}-07$ & 13.90 & $8.96 \mathrm{E}-10$ & $3.91 \mathrm{E}-09$ \\
\hline
\end{tabular}


Table A.3

Neutron Data

Detector 3 in Fig. 4

Detector Coordinates $(\mathrm{cm}): \quad x=39.0, y=0.0, z^{\prime}=94.0$

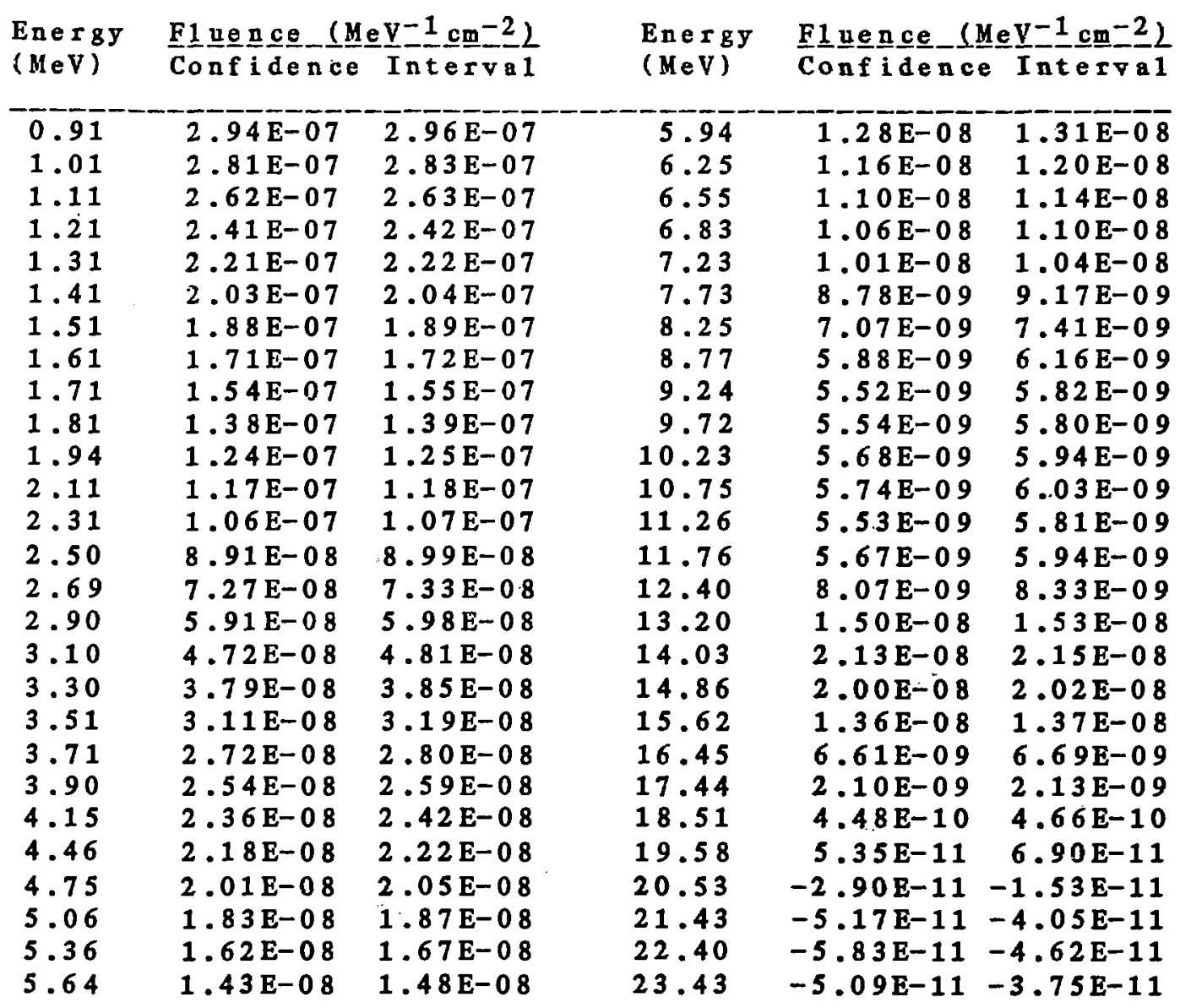


Table A.3 (Cont'd)

Gamma-Ray Data

Detector 3 in Fig. 4

Detector Coordinates (cm): $x=39.0, y=0.0, z^{\prime}=94.0$

\begin{tabular}{|c|c|c|c|c|c|}
\hline $\begin{array}{l}\text { Energy } \\
(\mathrm{MeV})\end{array}$ & 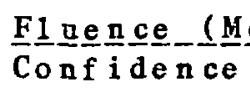 & $\frac{V}{\text { Int }}-\frac{1}{\cos }-\frac{2}{\mathrm{a}} \mathrm{L}$ & $\begin{array}{l}\text { Energy } \\
(\mathrm{MeV})\end{array}$ & 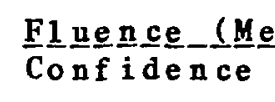 & 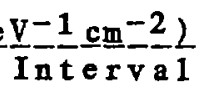 \\
\hline 0.72 & $4.02 \mathrm{E}-07$ & $6.59 \mathrm{E}-07$ & 3.93 & $8.71 \mathrm{E}-08$ & $9.09 \bar{E}-0 \overline{8}$ \\
\hline 0.76 & $6.00 \mathrm{E}-07$ & $7.03 \mathrm{E}-07$ & 4.06 & $7.72 \mathrm{E}-08$ & $8.13 \mathrm{E}-08$ \\
\hline 0.80 & $6.89 \mathrm{E}-07$ & $7.17 \mathrm{E}-07$ & 4.19 & $7.45 \mathrm{E}-08$ & $7.86 \mathrm{E}-08$ \\
\hline 0.84 & $6.87 \mathrm{E}-07$ & $6.97 \mathrm{E}-07$ & 4.31 & $7.70 \mathrm{E}-08$ & $8.19 \mathrm{E}-08$ \\
\hline 0.88 & $6.36 \mathrm{E}-07$ & $6.44 \mathrm{E}-07$ & 4.44 & $8.05 \mathrm{E}-08$ & $8.65 \mathrm{E}-08$ \\
\hline 0.92 & $5.68 \mathrm{E}-07$ & $5.76 \mathrm{E}-07$ & 4.57 & $8.34 \mathrm{E}-08$ & $8.99 E-08$ \\
\hline 0.96 & $5.05 \mathrm{E}-07$ & $5.11 E-07$ & 4.70 & $8.59 \mathrm{E}-08$ & $9.22 \mathrm{E}-08$ \\
\hline 1.00 & $4.58 \mathrm{E}-07$ & $4.64 \mathrm{E}-07$ & 4.83 & $8.66 \mathrm{E}-08$ & $9.30 \mathrm{E}-08$ \\
\hline 1.04 & $4.33 \mathrm{E}-07$ & $4.38 \mathrm{E}-07$ & 4.99 & $8.07 \mathrm{E}-08$ & $8.84 \mathrm{E}-08$ \\
\hline 1.08 & $4.23 \mathrm{E}-07$ & $4.27 \mathrm{E}-07$ & 5.15 & $6.40 \mathrm{E}-08$ & $7.26 \mathrm{E}-08$ \\
\hline 1.13 & $4.20 \mathrm{E}-07$ & $4.24 \mathrm{E}-07$ & 5.32 & $4.54 E-08$ & $5.39 \mathrm{E}-08$ \\
\hline 1.17 & $4.16 \mathrm{E}-07$ & $4.21 \mathrm{E}-07$ & 5.50 & $3.92 \mathrm{E}-08$ & $4.86 E-08$ \\
\hline 1.22 & $4.07 \mathrm{E}-07$ & $4.13 \mathrm{E}-07$ & 5.67 & $5.54 \mathrm{E}-08$ & $6.55 \mathrm{E}-08$ \\
\hline 1.28 & $3.90 \mathrm{E}-07$ & $3.97 \mathrm{E}-07$ & 5.83 & $8.77 \mathrm{E}-08$ & $9.71 \mathrm{E}-08$ \\
\hline 1.33 & $3.71 \mathrm{E}-07$ & $3.76 \mathrm{E}-07$ & 6.00 & $1.20 \mathrm{E}-07$ & $1.28 \mathrm{E}-07$ \\
\hline 1.38 & $3.51 \mathrm{E}-07$ & $3.56 \mathrm{E}-07$ & 6.18 & $1.37 \mathrm{E}-07$ & $1.46 \mathrm{E}-07$ \\
\hline 1.42 & $3.33 \mathrm{E}-07$ & $3.37 \mathrm{E}-07$ & 6.35 & $1.34 \mathrm{E}-07$ & $1.42 \mathrm{E}-07$ \\
\hline 1.47 & $3.16 \mathrm{E}-07$ & $3.20 \mathrm{E}-07$ & 6.51 & $1.15 \mathrm{E}-07$ & $1.22 \mathrm{E}-07$ \\
\hline 1.53 & $3.01 \mathrm{E}-07$ & $3.05 \mathrm{E}-07$ & 6.70 & $8.98 \mathrm{E}-08$ & $9.54 \mathrm{E}-08$ \\
\hline 1.58 & $2.91 \mathrm{E}-07$ & $2.96 \mathrm{E}-07$ & 6.90 & $7.18 \mathrm{E}-08$ & $7.68 \mathrm{E}-08$ \\
\hline 1.64 & $2.92 E-07$ & $2.97 E-07$ & 7.10 & $6.79 E-08$ & $7.26 \mathrm{E}-08$ \\
\hline $\begin{array}{l}1.72 \\
1.80\end{array}$ & $\begin{array}{l}3.13 \mathrm{E}-07 \\
3.47 \mathrm{E}-07\end{array}$ & $\begin{array}{l}3.17 \mathrm{E}-07 \\
3.51 \mathrm{E}-07\end{array}$ & $\begin{array}{l}7.30 \\
7.50\end{array}$ & $\begin{array}{l}7.21 \mathrm{E}-08 \\
7.46 \mathrm{E}-08\end{array}$ & $\begin{array}{l}7.64 \mathrm{E}-08 \\
7.84 \mathrm{E}-08\end{array}$ \\
\hline 1.88 & $3.82 \mathrm{E}-07$ & $3.86 E-07$ & 7.7 .0 & $6.83 E-08$ & $7.17 \mathrm{E}-08$ \\
\hline 1.96 & $4.01 \mathrm{E}-07$ & $4.04 \mathrm{E}-07$ & 7.90 & $5.33 \mathrm{E}-08$ & $5.64 \mathrm{E}-08$ \\
\hline 2.04 & $3.99 \mathrm{E}-07$ & $4.03 \mathrm{E}-07$ & 8.10 & $3.47 \mathrm{E}-08$ & $3.74 E-08$ \\
\hline 2.12 & $3.87 \mathrm{E}-07$ & $3.91 \mathrm{E}-07$ & 8.30 & $1.84 \mathrm{E}-08$ & $2.07 E-08$ \\
\hline 2.20 & $3.64 \mathrm{E}-07$ & $3.67 \mathrm{E}-07$ & 8.50 & $8.03 E-09$ & $9.99 \mathrm{E}-09$ \\
\hline 2.28 & $3.21 \mathrm{E}-07$ & $3.24 \mathrm{E}-07$ & 8.73 & $3.57 \mathrm{E}-09$ & $5.29 \mathrm{E}-09$ \\
\hline 2.36 & $2.64 \mathrm{E}-07$ & $2.68 \mathrm{E}-07$ & 8.98 & $4.08 \mathrm{E}-09$ & $5.56 \mathrm{E}-09$ \\
\hline 2.45 & $2.03 \mathrm{E}-07$ & $2.06 \mathrm{E}-07$ & 9.23 & $5.76 \mathrm{E}-09$ & $7.01 \mathrm{E}-09$ \\
\hline 2.55 & $1.58 E-07$ & $1.61 \mathrm{E}-07$ & 9.48 & $5.83 \mathrm{E}-09$ & $6.85 \mathrm{E}-09$ \\
\hline 2.65 & $1.39 \mathrm{E}-07$ & $1.42 \mathrm{E}-07$ & 9.73 & $4.05 \mathrm{E}-09$ & $4.93 \mathrm{E}-09$ \\
\hline 2.75 & $1.31 \mathrm{E}-07$ & $1.34 \mathrm{E}-07$ & 9.98 & $1.57 \mathrm{E}-09$ & $2.40 \mathrm{E}-09$ \\
\hline 2.85 & $1.25 \mathrm{E}-07$ & $1.27 \mathrm{E}-07$ & 10.30 & $-8.03 E-10$ & $3.43 \mathrm{E}-11$ \\
\hline 2.95 & $1.16 \mathrm{E}-07$ & $1.19 \mathrm{E}-07$ & 10.70 & $-1.49 \mathrm{E}-09$ & $-6.32 \mathrm{E}-10$ \\
\hline 3.05 & $1.06 E-07$ & $1.09 \mathrm{E}-07$ & 11.10 & $-8.27 E-10$ & $-1.73 E-11$ \\
\hline 3.15 & $9.91 \mathrm{E}-08$ & $1.02 E-07$ & 11.50 & $-8.49 E-11$ & $5.21 \mathrm{E}-10$ \\
\hline 3.25 & $9.50 \mathrm{E}-08$ & $9.83 \mathrm{E}-08$ & 11.90 & $2.04 \mathrm{E}-10$ & $6.70 \mathrm{E}-10$ \\
\hline 3.35 & $9.51 \mathrm{E}-08$ & $9.83 \mathrm{E}-08$ & 12.30 & $1.99 \mathrm{E}-10$ & $7.28 \mathrm{E}-10$ \\
\hline 3.45 & $9.85 \mathrm{E}-08$ & $1.02 \mathrm{E}-07$ & 12.70 & $2.26 E-10$ & $7.55 \mathrm{E}-10$ \\
\hline 3.55 & $1.03 \mathrm{E}-07$ & $1.06 \mathrm{E}-07$ & 13.10 & $2.11 \mathrm{E}-10$ & $6.57 \mathrm{E}-10$ \\
\hline 3.66 & $1.04 \mathrm{E}-07$ & $1.08 \mathrm{E}-07$ & 13.50 & $1.47 \mathrm{E}-10$ & 5. $31 \mathrm{E}-10$ \\
\hline 3.79 & $9.85 \mathrm{E}-08$ & $1.02 \mathrm{E}-07$ & 13.90 & $8.71 \mathrm{E}-11$ & $4.01 \mathrm{E}-10$ \\
\hline
\end{tabular}


Table A. 4

Neutron Data

Detector 4 in Fig. 4

Detector Coordinates $(\mathrm{cm}): x=59.0, y=0.0, z^{\prime}=94.0$

\begin{tabular}{|c|c|c|c|c|c|}
\hline \multirow{2}{*}{$\begin{array}{l}\text { Energy } \\
(\mathrm{MeV})\end{array}$} & \multicolumn{2}{|c|}{ 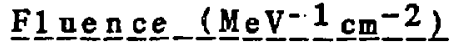 } & \multirow{2}{*}{$\begin{array}{l}\text { Energy } \\
(\mathrm{MeV})\end{array}$} & \multicolumn{2}{|c|}{$\underline{F} \underline{\underline{n}} \mathrm{~g} \underline{\mathrm{c}} \mathrm{c}$} \\
\hline & Confidence & Interva 1 & & Confidence & Interval \\
\hline 0.91 & $1.12 \mathrm{E}-07$ & $1.13 \mathrm{E}-07$ & 5.94 & $6.11 \mathrm{E}-09$ & $6.33 \mathrm{E}-09$ \\
\hline 1.01 & $1.06 \mathrm{E}-07$ & $1.07 \mathrm{E}-07$ & 6.25 & $5.66 \mathrm{E}-09$ & $5.90 \mathrm{E}-09$ \\
\hline 1.11 & $9.85 \mathrm{E}-08$ & $9.95 \mathrm{E}-08$ & 6.55 & $5.40 \mathrm{E}-09$ & $5.65 \mathrm{E}-09$ \\
\hline 1.21 & $9.14 \mathrm{E}-08$ & $9.23 \mathrm{E}-08$ & 6.83 & $5.18 \mathrm{E}-09$ & $5.39 \mathrm{E}-09$ \\
\hline 1.31 & $8.56 \mathrm{E}-08$ & $8.63 \mathrm{E}-08$ & 7.23 & $4.82 \mathrm{E}-09$ & $5.01 \mathrm{E}-09$ \\
\hline 1.41 & $8.07 \mathrm{E}-08$ & $8.14 \mathrm{E}-08$ & 7.73 & $4.10 \mathrm{E}-09$ & $4.32 \mathrm{E}-09$ \\
\hline 1.51 & $7.58 \mathrm{E}-08$ & $7.66 \mathrm{E}-08$ & 8.25 & $3.14 \mathrm{E}-09$ & $3.33 \mathrm{E}-09$ \\
\hline 1.61 & $6.98 \mathrm{E}-08$ & $7.04 E-08$ & 8.77 & $2.65 \mathrm{E}-09$ & $2.81 \mathrm{E}-09$ \\
\hline 1.71 & $6.32 \mathrm{E}-08$ & $6.38 \mathrm{E}-08$ & 9.24 & $2.55 \mathrm{E}-09$ & $2.71 \mathrm{E}-09$ \\
\hline 1.81 & $5.76 \mathrm{E}-08$ & $5.84 \mathrm{E}-08$ & 9.72 & $2.45 \mathrm{E}-09$ & $2.59 \mathrm{E}-09$ \\
\hline 1.94 & $5.40 \mathrm{E}-08$ & $5.47 E-08$ & 10.23 & $2.33 \mathrm{E}-09$ & $2.48 \mathrm{E}-09$ \\
\hline 2.11 & $5.40 \mathrm{E}-08$ & $5.45 \mathrm{E}-08$ & 10.75 & $2.30 \mathrm{E}-09$ & $2.45 \mathrm{E}-09$ \\
\hline 2.31 & $5.11 \mathrm{E}-08$ & $5.16 \mathrm{E}-08$ & 11.26 & $2.34 \mathrm{E}-09$ & $2.49 \mathrm{E}-09$ \\
\hline 2.50 & $4.27 \mathrm{E}-08$ & $4.31 \mathrm{E}-08$ & 11.76 & $2.65 \mathrm{E}-09$ & $2.79 \mathrm{E}-09$ \\
\hline 2.69 & $3.37 \mathrm{E}-08$ & $3.40 \mathrm{E}-08$ & 12.40 & $3.77 \mathrm{E}-09$ & $3.91 \mathrm{E}-09$ \\
\hline 2.90 & $2.63 \mathrm{E}-08$ & $2.67 \mathrm{E}-08$ & 13.20 & $6.05 \mathrm{E}-09$ & $6.16 \mathrm{E}-09$ \\
\hline 3.10 & $2.03 \mathrm{E}-08$ & $2.08 \mathrm{E}-08$ & 14.03 & $7.50 \mathrm{E}-09$ & $7.59 \mathrm{E}-09$ \\
\hline 3.30 & $1.58 \mathrm{E}-08$ & $1.61 \mathrm{E}-08$ & 14.86 & $6.46 \mathrm{E}-09$ & $6.54 E-09$ \\
\hline 3.51 & $1.27 \mathrm{E}-08$ & $1.31 \mathrm{E}-08$ & 15.62 & $4.16 E-09$ & $4.21 E-09$ \\
\hline 3.71 & $1.11 \mathrm{E}-08$ & $1.16 \mathrm{E}-08$ & 16.45 & $1.91 \mathrm{E}-0.9$ & $1.94 \mathrm{E}-09$ \\
\hline 3.90 & $1.06 \mathrm{E}-08$ & $1.10 \mathrm{E}-08$ & 17.44 & $5.62 \mathrm{E}-10$ & $5.78 \mathrm{E}-10$ \\
\hline & & $1.06 \mathrm{E}-08$ & 18.51 & $1.07 \mathrm{E}-10$ & $1.14 \mathrm{E}-10$ \\
\hline 4.46 & $9.70 \mathrm{E}-09$ & $9.92 \mathrm{E}-09$ & 19.58 & $6.90 \mathrm{E}-12$ & $1.28 \mathrm{E}-11$ \\
\hline 4.75 & $9.18 \mathrm{E}-09$ & $9.43 \mathrm{E}-09$ & 20.53 & $-1.24 \mathrm{E}-11$ & $-7.23 E-12$ \\
\hline 5.06 & $8.47 \mathrm{E}-09$ & $8.73 \mathrm{E}-09$ & 21.43 & $-1.77 E-11$ & $-1.34 E-11$ \\
\hline 5.36 & $7.53 \mathrm{E}-09$ & $7.82 \mathrm{E}-09$ & 22.40 & $-1.93 \mathrm{E}-11$ & $-1.47 \mathrm{E}-11$ \\
\hline 5.64 & $6.74 \mathrm{E}-09$ & $7.00 \mathrm{E}-09$ & 23.43 & $-1.68 \mathrm{E}-11$ & $-1.17 \mathrm{E}-11$ \\
\hline
\end{tabular}


Table A.4 (Cont'd)

Gamma-Ray Data

Detector 4 in Fig. 4

Detector Coordinates $(\mathrm{cm}): x=59.0, y=0.0, z^{\prime}=94.0$

\begin{tabular}{|c|c|c|c|c|c|}
\hline $\begin{array}{l}\text { Energy } \\
(\mathrm{MeV})\end{array}$ & \multicolumn{2}{|c|}{ 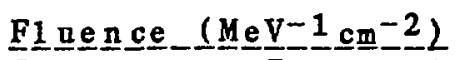 } & $\begin{array}{l}\text { Energy } \\
(\mathrm{MeV})\end{array}$ & \multicolumn{2}{|c|}{ Fl 므를 $\left(M e V^{-1} \mathrm{~cm}^{-2}\right)$} \\
\hline 0.72 & $1.78 \mathrm{E}-07$ & $3.29 \mathrm{E}-07$ & 3.93 & $5.31 \mathrm{E}-08$ & $5.58 \mathrm{E}-08$ \\
\hline & $3.03 \mathrm{E}-07$ & $3.63 \mathrm{E}-07$ & & $4.84 \mathrm{E}-08$ & $5.11 \mathrm{E}-08$ \\
\hline 0.80 & $3.54 \mathrm{E}-07$ & $3.71 \mathrm{E}-07$ & 4.19 & $4.79 \mathrm{E}-08$ & $5.07 \mathrm{E}-08$ \\
\hline 0.84 & $3.50 \mathrm{E}-07$ & $3.57 \mathrm{E}-07$ & 4.31 & $4.98 \mathrm{E}-08$ & $5.32 \mathrm{E}-08$ \\
\hline 0.88 & $3.27 \mathrm{E}-07$ & $3.33 \mathrm{E}-07$ & 4.44 & $5.14 \mathrm{E}-08$ & $5.55 \mathrm{E}-08$ \\
\hline 0.92 & $3.01 \mathrm{E}-07$ & $3.07 \mathrm{E}-07$ & 4.57 & $5.17 \mathrm{E}-08$ & $5.62 . E-08$ \\
\hline 0.96 & $2.79 \mathrm{E}-07$ & $2.85 \mathrm{E}-07$ & 4.70 & $5.19 \mathrm{E}-08$ & $5.63 \mathrm{E}-08$ \\
\hline 1.00 & $2.63 \mathrm{E}-07$ & $2.68 \mathrm{E}-07$ & 4.83 & $5.23 \mathrm{E}-08$ & $5.67 \mathrm{E}-08$ \\
\hline 1.04 & $2.54 \mathrm{E}-07$ & $2.58 \mathrm{E}-07$ & 4.99 & $5.05 \mathrm{E}-08$ & $5.59 \mathrm{E}-08$ \\
\hline 1.08 & $2.49 \mathrm{E}-07$ & $2.53 \mathrm{E}-07$ & 5.15 & $4.31 E-08$ & $4.90 E-08$ \\
\hline 1.13 & $2.47 \mathrm{E}-07$ & $2.50 \mathrm{E}-07$ & 5.32 & $3.20 \mathrm{E}-08$ & $3.78 \mathrm{E}-08$ \\
\hline 1.17 & $2.43 \mathrm{E}-07$ & $2.46 \mathrm{E}-07$ & 5.50 & $2.54 \mathrm{E}-08$ & $3.20 \mathrm{E}-08$ \\
\hline 1.22 & $2.36 \mathrm{E}-07$ & $2.40 \mathrm{E}-07$ & 5.67 & $3.25 \mathrm{E}-08$ & $3.95 \mathrm{E}-08$ \\
\hline 1.28 & $2.28 \mathrm{E}-07$ & $2.32 \mathrm{E}-07$ & 5.83 & $5.29 \mathrm{E}-08$ & $5.92 \mathrm{E}-08$ \\
\hline 1.33 & $2.18 \mathrm{E}-07$ & $2.22 \mathrm{E}-0.7$ & 6.00 & $7.61 \mathrm{E}-08$ & $8.17 \mathrm{E}-08$ \\
\hline 1.38 & $2.08 \mathrm{E}-07$ & $2.11 \mathrm{E}-07$ & 6.18 & $9.01 \mathrm{E}-08$ & $9.60 \mathrm{E}-08$ \\
\hline 1.42 & $1.99 \mathrm{E}-07$ & $2.02 \mathrm{E}-07$ & 6.35 & $8.95 \mathrm{E}-08$ & $9.50 \mathrm{E}-08$ \\
\hline 1.47 & $1.93 \mathrm{E}-07$ & $1.96 \mathrm{E}-07$ & 6.51 & $7.70 \mathrm{E}-08$ & $8.17 \mathrm{E}-0 \mathrm{~g}$ \\
\hline 1.53 & $1.87 \mathrm{E}-07$ & $1.90 \mathrm{E}-07$ & 6.70 & $5.92 \mathrm{E}-08$ & $6.30 \mathrm{E}-08$ \\
\hline 1.58 & $1.83 \mathrm{E}-07$ & $1.86 \mathrm{E}-07$ & 6.90 & $4.53 \mathrm{E}-0.8$ & $4.87 E-08$ \\
\hline 1.64 & $1.81 \mathrm{E}-07$ & $1.84 \mathrm{E}-07$ & 7.10 & $4.13 \mathrm{E}-08$ & $4.45 \mathrm{E}-08$ \\
\hline $\begin{array}{l}1.72 \\
1.80\end{array}$ & $\begin{array}{l}1.91 \mathrm{E}-07 \\
2.13 \mathrm{E}-07\end{array}$ & $\begin{array}{l}1.94 \mathrm{E}-07 \\
2.15 \mathrm{E}-07\end{array}$ & & $\begin{array}{l}4.31 \mathrm{E}-08 \\
4.34 \mathrm{E}-08\end{array}$ & $\begin{array}{l}4.59 \mathrm{E}-08 \\
4.60 \mathrm{E}-08\end{array}$ \\
\hline 1.88 & $2.36 \mathrm{E}-07$ & $2.38 \mathrm{E}-07$ & 7.70 & $3.83 \mathrm{E}-08$ & $4.05 \mathrm{E}-08$ \\
\hline 1.96 & $2.47 \mathrm{E}-07$ & $2.50 \mathrm{E}-07$ & 7.90 & $2.83 \mathrm{E}-08$ & $3.03 \mathrm{E}-08$ \\
\hline 2.04 & $2.47 \mathrm{E}-07$ & $2.49 \mathrm{E}-07$ & 8.10 & $1.72 \mathrm{E}-08$ & $1.89 \mathrm{E}-08$ \\
\hline 2.12 & $2.38 \mathrm{E}-07$ & $2.41 \mathrm{E}-07$ & 8.30 & $8.45 \mathrm{E}-09$ & $1.00 \mathrm{E}-08$ \\
\hline 2.20 & $2.21 \mathrm{E}-07$ & $2.23 \mathrm{E}-0 ?$ & 8.50 & $3.79 \mathrm{E}-09$ & $5.11 \mathrm{E}-09$ \\
\hline 2.28 & $1.92 \mathrm{E}-07$ & $1.95 \mathrm{E}-07$ & 8.73 & $2.54 \mathrm{E}-09$ & $3.65 \mathrm{E}-09$ \\
\hline 2.36 & $1.57 \mathrm{E}-07$ & $1.59 \mathrm{E}-07$ & 3. 98 & 3. $37 \mathrm{E}-09$ & $4.34 E-09$ \\
\hline 2.45 & $1.22 \mathrm{E}-07$ & $1.24 \mathrm{E}-07$ & 9.23 & $3.90 \mathrm{E}-09$ & $4.70 \mathrm{E}-09$ \\
\hline 2.55 & $9.72 \mathrm{E}-08$ & $9.92 \mathrm{E}-08$ & 9.48 & $3.09 \mathrm{E}-09$ & $3.79 \mathrm{E}-09$ \\
\hline 2.65 & $8.53 \mathrm{E}-08$ & $8.74 \mathrm{E}-08$ & 9 . & $1.53 \mathrm{E}-09$ & $2.14 \mathrm{E}-09$ \\
\hline 2.75 & $8.01 \mathrm{E}-08$ & $8.22 \mathrm{E}-08$ & 9.98 & $1.05 \mathrm{E}-10$ & $6.37 \mathrm{E}-10$ \\
\hline 2.85 & $7.64 \mathrm{E}-08$ & $7.84 \mathrm{E}-08$ & 10.30 & $-7.27 \mathrm{E}-10$ & $-2.06 \mathrm{E}-10$ \\
\hline 2.95 & $7.13 \mathrm{E}-08$ & $7.33 \mathrm{E}-08$ & 10.70 & $-4.90 \mathrm{E}-10$ & $4.98 \mathrm{E}-11$ \\
\hline 3.05 & $6.49 \mathrm{E}-08$ & $6.70 \mathrm{E}-08$ & 11.10 & $1.25 \mathrm{E}-11$ & $5.50 \mathrm{E}-10$ \\
\hline 3.15 & $5.97 \mathrm{E}-08$ & $6.18 \mathrm{E}-08$ & 11.50 & $1.99 \mathrm{E}-10$ & $6.73 \mathrm{E}-10$ \\
\hline 3.25 & $5.75 \mathrm{E}-08$ & $5.99 \mathrm{E}-08$ & 11.90 & $1.38 \mathrm{E}-10$ & $4.96 \mathrm{E}-10$ \\
\hline 3.35 & $5.87 \mathrm{E}-08$ & $6.10 E-08$ & 12.30 & $-1.89 z-11$ & $3.09 \mathrm{E}-10$ \\
\hline 3.45 & $6.16 \mathrm{E}-08$ & $6.38 \mathrm{E}-08$ & 12.70 & $-7.04 \mathrm{E}-11$ & $2.81 \mathrm{E}-10$ \\
\hline 3.55 & $6.38 \mathrm{E}-08$ & $6.62 \mathrm{E}-08$ & 13.10 & $-3.14 E-11$ & $2.93 \mathrm{E}-10$ \\
\hline 3.66 & $6.37 \mathrm{E}-08$ & $6.63 \mathrm{E}-08$ & 13.50 & $-4.14 E-11$ & $2.48 \mathrm{E}-10$ \\
\hline 3.79 & $5.95 \mathrm{E}-08$ & $6.22 \mathrm{E}-08$ & 13.90 & $-3.58 E-11$ & $2.03 \mathrm{E}-10$ \\
\hline
\end{tabular}


Table A.5

Neutron Data

Detector 5 in Fig. 4

Detector Coordinates $(\mathrm{cm}): \mathrm{x}=0.0, y=0.0, \mathrm{z}^{\prime}=121.0$

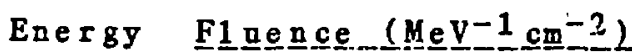
(MeV) Confidence Interva1

\begin{tabular}{lll}
\hline 0.91 & $2.29 \mathrm{E}-06$ & $2.32 \mathrm{E}-06$ \\
1.01 & $2.24 \mathrm{E}-00$ & $2.27 \mathrm{E}-06$ \\
1.11 & $1.97 \mathrm{E}-06$ & $1.99 \mathrm{E}-06$ \\
1.21 & $1.77 \mathrm{E}-06$ & $1.80 \mathrm{E}-06$ \\
1.31 & $1.75 \mathrm{E}-06$ & $1.77 \mathrm{E}-06$ \\
1.41 & $1.80 \mathrm{E}-06$ & $1.82 \mathrm{E}-06$ \\
1.51 & $1.76 \mathrm{E}-06$ & $1.78 \mathrm{E}-06$ \\
1.61 & $1.58 \mathrm{E}-06$ & $1.60 \mathrm{E}-06$ \\
1.71 & $1.32 \mathrm{E}-06$ & $1.33 \mathrm{E}-06$ \\
1.81 & $1.06 \mathrm{E}-06$ & $1.08 \mathrm{E}-06$ \\
1.94 & $8.57 \mathrm{E}-07$ & $8.76 \mathrm{E}-07$ \\
2.11 & $7.99 \mathrm{E}-07$ & $8.12 \mathrm{E}-07$ \\
2.31 & $7.36 \mathrm{E}-07$ & $7.51 \mathrm{E}-07$ \\
2.50 & $5.75 \mathrm{E}-07$ & $5.86 \mathrm{E}-07$ \\
2.69 & $4.76 \mathrm{E}-07$ & $4.86 \mathrm{E}-07$ \\
2.90 & $5.22 \mathrm{E}-07$ & $5.33 \mathrm{E}-07$ \\
3.10 & $5.42 \mathrm{E}-07$ & $5.57 \mathrm{E}-07$ \\
3.30 & $4.51 \mathrm{E}-07$ & $4.61 \mathrm{E}-07$ \\
3.51 & $3.33 \mathrm{E}-07$ & $3.45 \mathrm{E}-07$ \\
3.71 & $2.77 \mathrm{E}-07$ & $2.91 \mathrm{E}-07$ \\
3.90 & $2.66 \mathrm{E}-07$ & $2.75 \mathrm{E}-07$ \\
4.15 & $2.42 \mathrm{E}-07$ & $2.52 \mathrm{E}-07$ \\
4.46 & $1.94 \mathrm{E}-07$ & $2.00 \mathrm{E}-07$ \\
4.75 & $1.58 \mathrm{E}-07$ & $1.66 \mathrm{E}-07$ \\
5.06 & $1.34 \mathrm{E}-07$ & $1.43 \mathrm{E}-07$ \\
5.36 & $1.20 \mathrm{E}-07$ & $1.30 \mathrm{E}-07$ \\
5.64 & $1.21 \mathrm{E}-07$ & $1.29 \mathrm{E}-07$
\end{tabular}

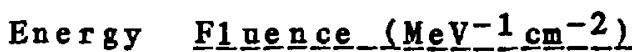
(MeV) Confidence Interval

$5.94 \quad 1.16 \mathrm{E}-07-1.23 \mathrm{E}-07$

$6.25 \quad 1.00 \mathrm{E}-07 \quad 1.09 \mathrm{E}-07$

$6.55 \quad 9.41 \mathrm{E}-08 \quad 1.03 \mathrm{E}-07$

$6.83 \quad 9.42 \mathrm{E}-08 \quad 1.02 \mathrm{E}-07$

$\begin{array}{lll}7.23 & 8.83 \mathrm{E}-08 & 9.58 \mathrm{E}-08\end{array}$

$\begin{array}{lll}7.73 & 6.54 \mathrm{E}-08 & 7.40 \mathrm{E}-08\end{array}$

$8.25 \quad 2.07 \mathrm{E}-08 \quad 2.87 \mathrm{E}-08$

$8.77 \quad 7.23 \mathrm{E}-09 \quad 1.44 \mathrm{E}-08$

$9.24 \quad 1.42 \mathrm{E}-08 \quad 2.20 \mathrm{E}-08$

$9.72 \quad 1.77 \mathrm{E}-08 \quad 2.49 \mathrm{E}-08$

$10.23 \quad 2.89 \mathrm{E}-08 \quad 3.65 \mathrm{E}-08$

$10.75 \quad 4.87 \mathrm{E}-08 \quad 5.71 \mathrm{E}-08$

$11.2655 .60 \mathrm{E}-08 \quad 6.44 \mathrm{E}-08$

$11.7655 .55 \mathrm{E}-08 \quad 6.38 \mathrm{E}-08$

$12.40 \quad 1.48 \mathrm{E}-07 \quad 1.56 \mathrm{E}-07$

$13.20 \quad 6.58 \mathrm{E}-07 \quad 6.66 \mathrm{E}-07$

$14.03 \quad 1.47 \mathrm{E}-06 \quad 1.48 \mathrm{E}-06$

$14.86 \quad 1.80 \mathrm{E}-06 \quad 1.81 \mathrm{E}-06$

$15.62 \quad 1.47 \mathrm{E}-06 \quad 1.47 \mathrm{E}-06$

$16.45 \quad 8.29 \mathrm{E}-07 \quad 8.32 \mathrm{E}-07$

$17.443 .02 \mathrm{E}-07 \quad 3.04 \mathrm{E}-07$

$18.517 .40 \mathrm{E}-08 \quad 7.46 \mathrm{E}-08$

$19.58 \quad 1.34 \mathrm{E}-08 \quad 1.36 \mathrm{E}-08$

$20.53 \quad 3.28 \mathrm{E}-10 \quad 4.60 \mathrm{E}-10$

$21.43-3.25 \mathrm{E}-09-3.16 \mathrm{E}-09$

$22.40-4.27 \mathrm{E}-09-4.17 \mathrm{E}-09$

$23.43-3.83 \mathrm{E}-09-3.73 \mathrm{E}-09$ 
Table A.5 (Cont'd)

Gamma-Ray Data

Detector 5 in Fig. 4

Detector Coordinates $(\mathrm{cm}): \quad x=0.0, y=0.0, z^{\prime}=121.0$

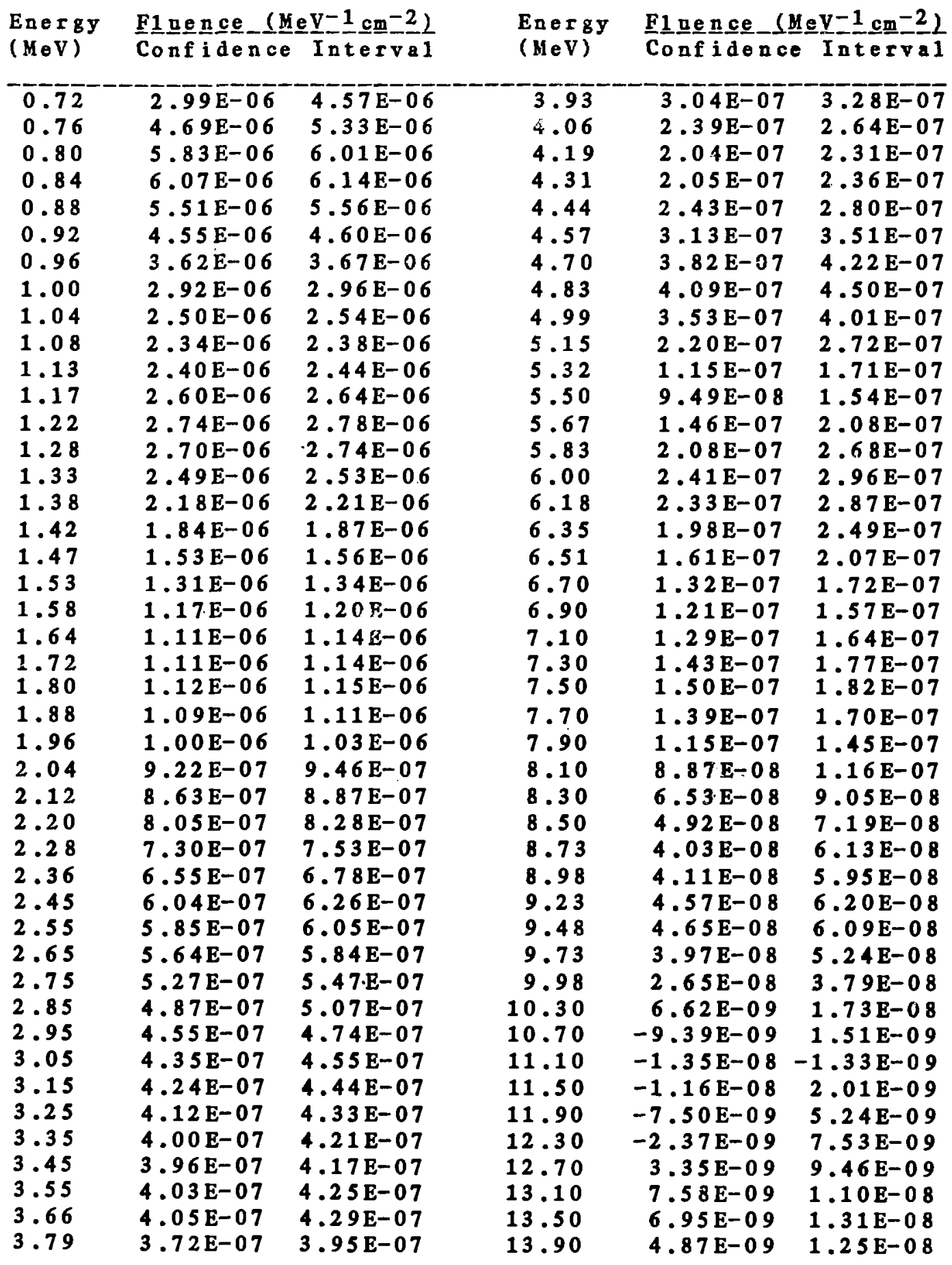


Table A. 6

Neutron Data

Detector 6 in Fig. 4

Detector Coordinates $(\mathrm{cm}): x=47.0, y=0.0, z^{\prime}=121.0$

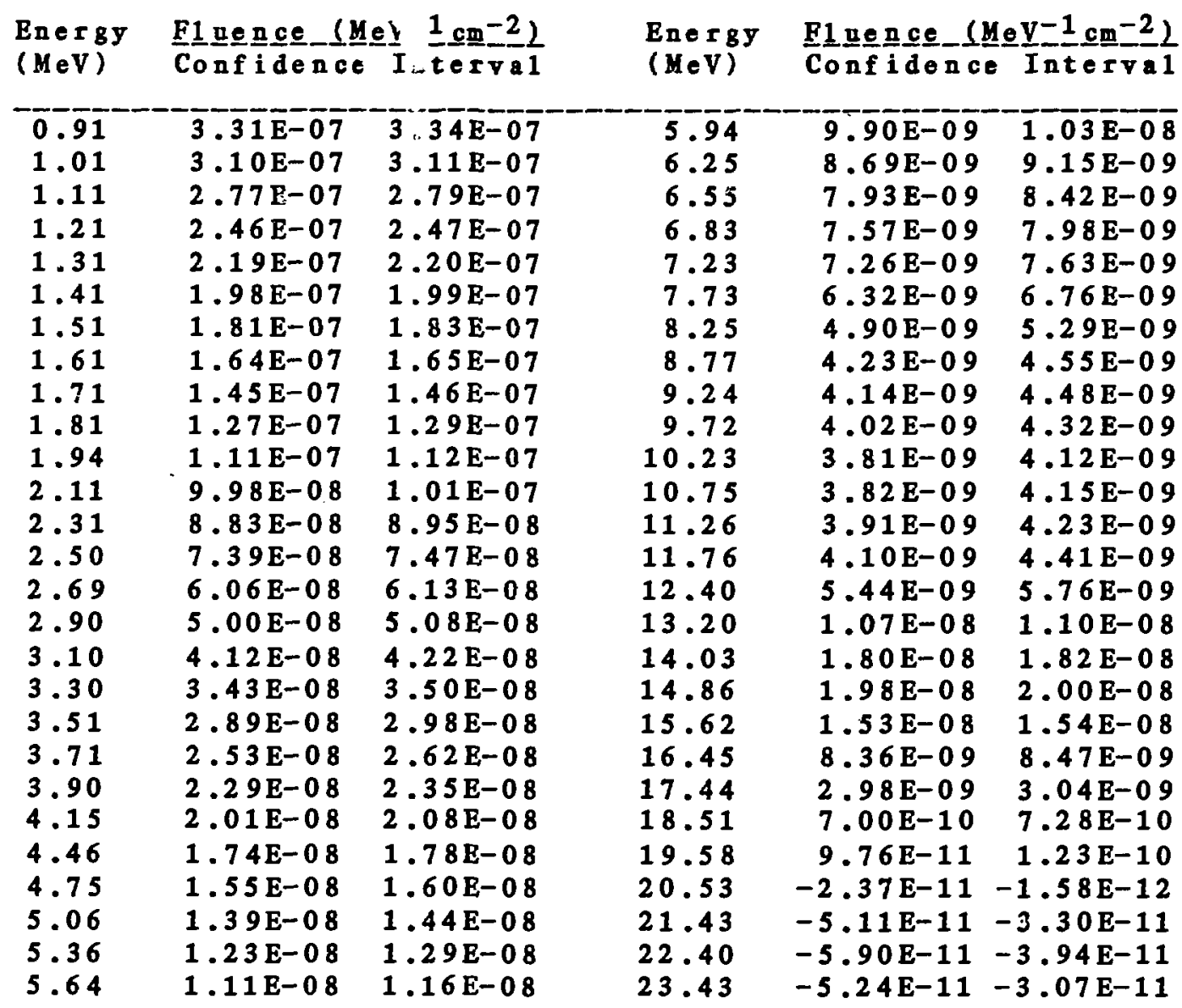


Table A.6 (Cont'd)

Gamma-Ray Data

Detector 6 in Fig. 4

Detector Coordinates $(\mathrm{cm}): x=47.0, y=0.0, z^{\prime}=121.0$

\begin{tabular}{|c|c|c|c|c|c|}
\hline \multirow{2}{*}{$\begin{array}{l}\text { Energy } \\
(M \operatorname{eV})\end{array}$} & \multicolumn{2}{|c|}{ 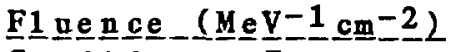 } & \multirow{2}{*}{$\begin{array}{l}\text { Energy } \\
(\mathrm{MeV})\end{array}$} & \multicolumn{2}{|c|}{ 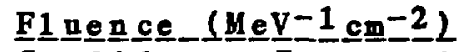 } \\
\hline & Confidence & Interva 1 & & Confidence & Interva 1 \\
\hline 0.72 & $2.95 \mathrm{E}-07$ & $4.91 \mathrm{E}-07$ & 3.93 & $5.65 \mathrm{E}-08$ & $6.00 \mathrm{E}-08$ \\
\hline 0.76 & $4.66 \mathrm{E}-07$ & $5.46 \mathrm{E}-07$ & 4.06 & $4.91 \mathrm{E}-08$ & $5.29 \mathrm{E}-08$ \\
\hline 0.80 & $5.63 \mathrm{E}-07$ & $5.86 \mathrm{E}-07$ & 4.19 & $4.46 \mathrm{E}-08$ & $4.86 \mathrm{E}-08$ \\
\hline 0.84 & $5.82 \mathrm{E}-07$ & $5.92 \mathrm{E}-07$ & 4.31 & $4.32 \mathrm{E}-0.8$ & $4.79 \mathrm{E}-08$ \\
\hline 0.88 & $5.43 \mathrm{E}-07$ & $5.50 \mathrm{E}-07$ & 4.44 & $4.45 \mathrm{E}-08$ & $5.01 \mathrm{E}-08$ \\
\hline 0.92 & $4.74 \mathrm{E}-07$ & $4.81 \mathrm{E}-07$ & 4.57 & $4.76 \mathrm{E}-08$ & $5.37 \mathrm{E}-0.8$ \\
\hline 0.96 & $4.04 E-07$ & $4.10 \mathrm{E}-07$ & 4.70 & $5.11 \mathrm{E}-08$ & $5.72 \mathrm{E}-08$ \\
\hline 1.00 & $3.47 \mathrm{E}-07$ & $3.52 \mathrm{E}-07$ & 4.83 & $5.32 \mathrm{E}-08$ & $5.95 \mathrm{E}-08$ \\
\hline 1.04 & $3.08 \mathrm{E}-07$ & $3.13 \mathrm{E}-07$ & 4.99 & $5.15 \mathrm{E}-08$ & $5.95 \mathrm{E}-08$ \\
\hline 1.08 & $2.90 \mathrm{E}-07$ & $2.94 \mathrm{E}-07$ & 5.15 & $4.38 \mathrm{E}-08$ & $5.22 \mathrm{E}-08$ \\
\hline 1.13 & $2.87 \mathrm{E}-07$ & $2.91 \mathrm{E}-07$ & 5.32 & $3.23 \mathrm{E}-08$ & $4.05 E-08$ \\
\hline 1.17 & $2.90 \mathrm{E}-07$ & $2.95 \mathrm{E}-07$ & 5.50 & $2.30 \mathrm{E}-08$ & $3.23 \mathrm{E}-08$ \\
\hline 1.22 & $2.89 \mathrm{E}-07$ & $2.95 \mathrm{E}-07$ & 5.67 & $2.54 \mathrm{E}-08$ & $3.54 \mathrm{E}-08$ \\
\hline 1.28 & $2.81 \mathrm{E}-07$ & $2.87 \mathrm{E}-07$ & 5.83 & $4.10 \mathrm{E}-0.8$ & $5.01 \mathrm{E}-08$ \\
\hline 1.33 & $2.68 \mathrm{E}-07$ & $2.73 \mathrm{E}-07$ & 6.00 & $6.12 \mathrm{E}-08$ & $6.95 \mathrm{E}-08$ \\
\hline 1.38 & $2.51 \mathrm{E}-07$ & $2.55 \mathrm{E}-07$ & 6.18 & $7.60 \mathrm{E}-08$ & $8.44 E-08$ \\
\hline 1.42 & $2.31 \mathrm{E}-07$ & $2.35 \mathrm{E}-07$ & 6.35 & $7.88 \mathrm{E}-08$ & $8.67 \mathrm{E}-08$ \\
\hline 1.47 & $2.12 \mathrm{E}-07$ & $2.16 \mathrm{E}-07$ & 6.51 & $7.04 \mathrm{E}-08$ & $7.75 \mathrm{E}-08$ \\
\hline 1.53 & $1.97 \mathrm{E}-07$ & $2.01 \mathrm{E}-07$ & 6.70 & $5: 60 \mathrm{E}-08$ & $6.16 \mathrm{E}-08$ \\
\hline 1.58 & 1. $87 \mathrm{E}-07$ & $1.91 \mathrm{E}-07$ & 6.90 & $4.33 \mathrm{E}-08$ & $4.85 \mathrm{E}-08$ \\
\hline $\begin{array}{l}1.64 \\
1.72 \\
1.80\end{array}$ & $\begin{array}{l}1.83 \mathrm{E}-07 \\
1.90 \mathrm{E}-07 \\
2.05 \mathrm{E}-07\end{array}$ & $\begin{array}{l}1.87 \mathrm{E}-07 \\
1.93 \mathrm{E}-07 \\
2.08 \mathrm{E}-07\end{array}$ & $\begin{array}{l}7.10 \\
7.30 \\
7.50\end{array}$ & $\begin{array}{l}3.93 \mathrm{E}-08 \\
4.20 \mathrm{E}-08 \\
4.56 \mathrm{E}-08\end{array}$ & $\begin{array}{l}4.42 \mathrm{E}-08 \\
4.64 \mathrm{E}-08 \\
4.96 \mathrm{E}-08\end{array}$ \\
\hline 1.88 & $2.21 \mathrm{E}-07$ & $2.25 \mathrm{E}-07$ & 7.70 & $4.48 \mathrm{E}-08$ & $4.87 \mathrm{E}-08$ \\
\hline 1.96 & $2.32 E-07$ & $2.36 \mathrm{E}-07$ & 7.90 & $3.86 E-08$ & $4.19 \mathrm{E}-08$ \\
\hline 2.04 & $2.33 \mathrm{E}-07$ & $2.37 \mathrm{E}-07$ & 8.10 & $2.84 \mathrm{E}-08$ & $3.14 \mathrm{E}-08$ \\
\hline 2.12 & $2.28 \mathrm{E}-07$ & $2.32 \mathrm{E}-07$ & 8.30 & $1.78 \mathrm{E}-08$ & $2.04 \mathrm{E}-08$ \\
\hline 2.20 & $2.19 \mathrm{E}-07$ & $2.23 \mathrm{E}-07$ & 8.50 & $9.62 \mathrm{E}-09$ & $1.19 \mathrm{E}-08$ \\
\hline 2.28 & $2.01 \mathrm{E}-07$ & $2.05 \mathrm{E}-07$ & 8.73 & $4.57 \mathrm{E}-09$ & $6.50 \mathrm{E}-09$ \\
\hline 2.36 & $1.74 E-07$ & $1.77 \mathrm{E}-07$ & 8.98 & $3.14 \mathrm{E}-09$ & $4.90 \mathrm{E}-09$ \\
\hline 2.45 & $1.38 \mathrm{E}-07$ & $1.41 \mathrm{E}-07$ & 9.23 & $3.60 \mathrm{E}-09$ & $5.03 \mathrm{E}-09$ \\
\hline 2.55 & $1.08 \mathrm{E}-07$ & $1.11 \mathrm{E}-07$ & 9.48 & $3.69 \mathrm{E}-09$ & $4.91 \mathrm{E}-09$ \\
\hline 2.65 & $9.31 \mathrm{E}-08$ & $9.59 \mathrm{E}-08$ & 9.73 & $2.74 \mathrm{E}-09$ & $3.85 \mathrm{E}-09$ \\
\hline 2.75 & $8.70 E-08$ & $8.99 \mathrm{E}-08$ & 9.98 & $1.15 \mathrm{E}-09$ & $2.28 \mathrm{E}-09$ \\
\hline 2.85 & $8.23 E-08$ & $8.51 \mathrm{E}-08$ & 10.30 & $-6.24 E-10$ & $6.27 \mathrm{E}-10$ \\
\hline 2.95 & $7.65 \mathrm{E}-08$ & $7.94 \mathrm{E}-08$ & 10.70 & $-1.36 \mathrm{E}-09$ & $-2.21 E-11$ \\
\hline 3.05 & $7.09 \mathrm{E}-08$ & $7.38 \mathrm{E}-08$ & 11.10 & $-8.90 \mathrm{E}-10$ & $2.56 \mathrm{E}-10$ \\
\hline 3.15 & $6.63 \mathrm{E}-08$ & $6.92 E-08$ & 11.50 & $-2.32 \mathrm{E}-10$ & $5.63 \mathrm{E}-10$ \\
\hline 3.25 & $6.27 \mathrm{E}-08$ & $6.59 \mathrm{E}-08$ & 11.90 & $1.06 \mathrm{E}-10$ & $7.96 \mathrm{E}-10$ \\
\hline 3.35 & $6.11 \mathrm{E}-08$ & $6.42 \mathrm{E}-08$ & 12.30 & $1.68 \mathrm{E}-10$ & $9.95 \mathrm{E}-10$ \\
\hline 3.45 & $6.21 \mathrm{E}-08$ & $6.51 \mathrm{E}-0.8$ & 12.70 & $2.17 \mathrm{E}-10$ & $9.74 \mathrm{E}-10$ \\
\hline 3.55 & $6.46 \mathrm{E}-08$ & $6.79 \mathrm{E}-08$ & 13.10 & $1.44 E-10$ & $8.15 E-10$ \\
\hline 3.66 & $6.63 E-08$ & $6.98 \mathrm{E}-08$ & 13.50 & $7.95 \mathrm{E}-11$ & $6.30 \mathrm{E}-10$ \\
\hline 3.79 & $6.35 E-08$ & $6.71 \mathrm{E}-08$ & 13.90 & $3.29 \mathrm{E}-11$ & $4.97 \mathrm{E}-10$ \\
\hline
\end{tabular}


Tab1e A. 7

Neutron Data

Detector 7 in Fig. 4

Detector Coordinates $(\mathrm{cm}): \mathrm{x}=59.0, \mathrm{y}=0.0, \mathrm{z}^{\prime}=121.0$

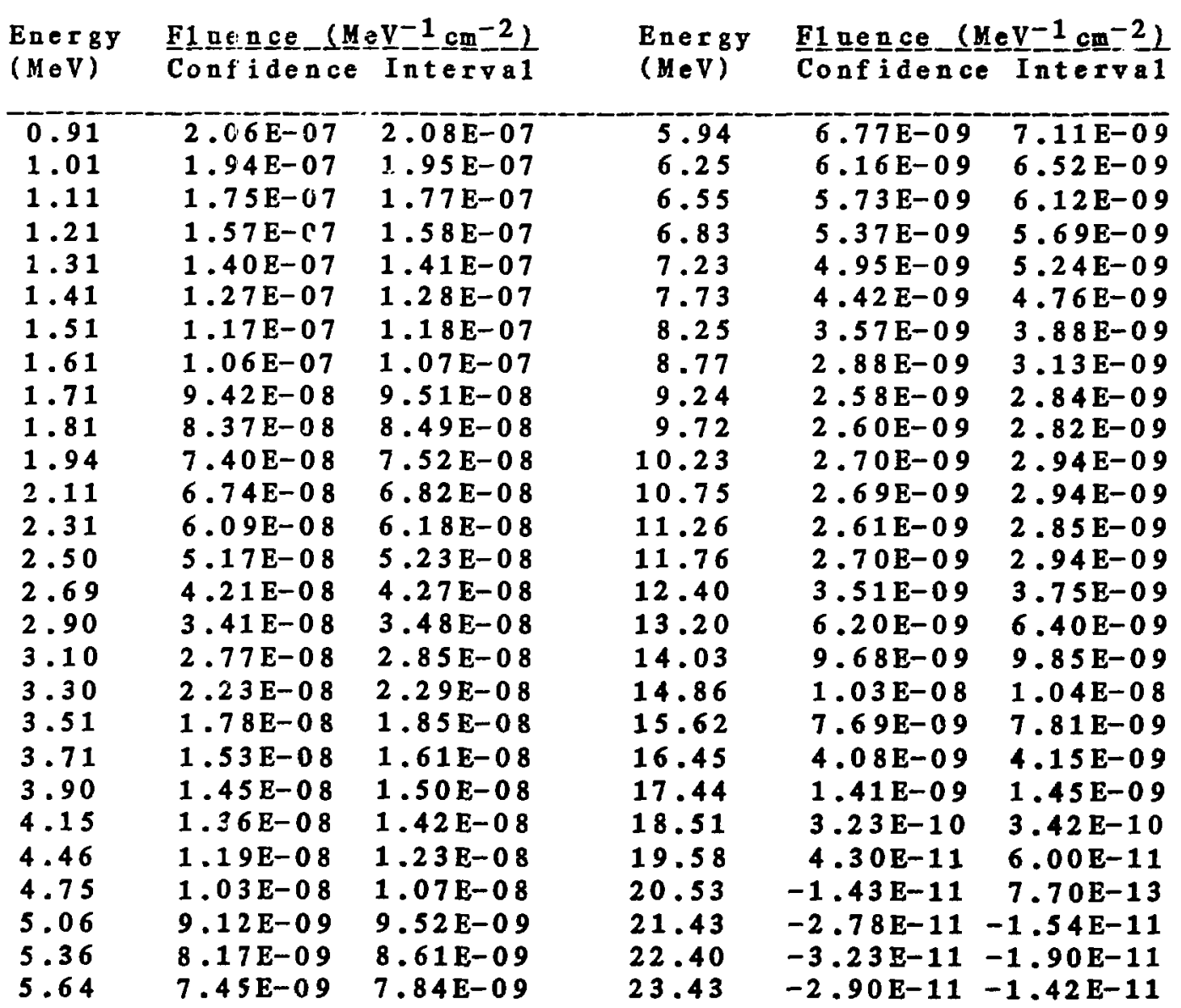


Table A.7 (Cont'd)

Gamma-Ray Data

Detector 7 in Fig. 4

Detector Coordinates $(\mathrm{cm}): x=59.0, y=0.0, z^{\prime}=121.0$

\begin{tabular}{|c|c|c|c|c|c|}
\hline \multirow{2}{*}{$\begin{array}{l}\text { Energy } \\
(\mathrm{HeV})\end{array}$} & \multicolumn{2}{|c|}{ F 1 ne $\mathrm{n} c \mathrm{e}-(\mathrm{MeV}-1 \mathrm{~cm}-2)$} & \multirow{2}{*}{$\begin{array}{l}\text { Energy } \\
(\mathrm{MeV})\end{array}$} & \multicolumn{2}{|c|}{ 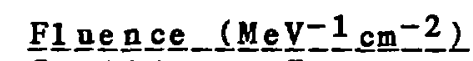 } \\
\hline & Conf idence & Interva 1 & & Confidence & Interval \\
\hline 0.72 & $2.24 \mathrm{E}-07$ & $3.76 \mathrm{E}-07$ & 3.93 & $4.70 \mathrm{E}-08$ & $5.03 \mathrm{E}-08$ \\
\hline 0.76 & $3.34 E-07$ & $3.96 \mathrm{E}-07$ & 4.06 & $4.04 E-08$ & $4.39 E-08$ \\
\hline 0.80 & $3.94 \mathrm{E}-07$ & $4.11 E-07$ & 4.19 & $3.53 \mathrm{E}-08$ & $3.90 \mathrm{E}-08$ \\
\hline 0.84 & $4.05 \mathrm{E}-07$ & $4.12 \mathrm{E}-07$ & 4.31 & $3.38 \mathrm{E}-08$ & $3.80 \mathrm{E}-08$ \\
\hline 0.88 & $3.79 \mathrm{E}-07$ & $3.85 \mathrm{E}-07$ & 4.4 .4 & $3.57 E-08$ & $4.07 \mathrm{E}-08$ \\
\hline 0.92 & $3.35 \mathrm{E}-07$ & $3.41 \mathrm{E}-07$ & 4.57 & $3.91 \mathrm{E}-08$ & $4.46 \mathrm{E}-08$ \\
\hline 0.96 & $2.89 \mathrm{E}-07$ & $2.95 \mathrm{E}-07$ & 4.70 & $4.21 \mathrm{E}-08$ & $4.77 \mathrm{E}-08$ \\
\hline 1.00 & $2.54 \mathrm{E}-07$ & $2.59 \mathrm{E}-07$ & 4.83 & $4.33 \mathrm{E}-08$ & $4.90 \mathrm{E}-08$ \\
\hline 1.04 & $2.32 \mathrm{E}-07$ & $2.36 \mathrm{E}-07$ & 4.99 & $4.13 \mathrm{E}-\cup 8$ & $4.82 \mathrm{E}-08$ \\
\hline 1.08 & $2.20 \mathrm{E}-07$ & $2.24 \mathrm{E}-07$ & 5.15 & $3.45 \mathrm{E}-08$ & $4.20 \mathrm{E}-08$ \\
\hline 1.13 & $2.17 \mathrm{E}-07$ & $2.21 \mathrm{E}-07$ & 5.32 & $2.46 \mathrm{E}-08$ & $3.21 \mathrm{E}-08$ \\
\hline 1.17 & $2.22 \mathrm{E}-07$ & $2.26 \mathrm{E}-07$ & 5.50 & $1.65 \mathrm{E}-08$ & $2.48 \mathrm{E}-0.8$ \\
\hline 1.22 & $2.25 \mathrm{E}-07$ & $2.30 \mathrm{E}-07$ & 5.67 & $1.84 \mathrm{E}-0.8$ & $2.72 \mathrm{E}-08$ \\
\hline 1.28 & $2.20 \mathrm{E}-07$ & $2.25 \mathrm{E}-07$ & 5.8 & $3.19 \mathrm{E}-08$ & $4.00 \mathrm{E}-08$ \\
\hline 1.33 & $2.09 \mathrm{E}-07$ & $2.13 \mathrm{E}-07$ & 6.00 & $4.99 \mathrm{E}-08$ & $5.73 \mathrm{E}-08$ \\
\hline 1.38 & $1.95 \mathrm{E}-07$ & $1.99 \mathrm{E}-07$ & 6.18 & $6.37 \mathrm{E}-08$ & $7.14 \mathrm{E}-08$ \\
\hline 1.42 & $1.81 \mathrm{E}-07$ & $1.85 \mathrm{E}-07$ & 6.35 & $6.73 E-08$ & $7.46 \mathrm{E}-08$ \\
\hline 1.47 & $1.67 \mathrm{E}-07$ & $1.70 \mathrm{E}-07$ & 6.51 & $6.10 \mathrm{E}-08$ & $6.72 \mathrm{E}-08$ \\
\hline 1.53 & $1.56 \mathrm{E}-07$ & $1.60 \mathrm{E}-07$ & 6.70 & $4.89 \mathrm{E}-08$ & $5.40 \mathrm{E}-08$ \\
\hline 1.58 & $1.51 \mathrm{E}-07$ & $1.54 \mathrm{E}-07$ & 6.90 & $3.81 \mathrm{E}-08$ & $4.28 \mathrm{E}-08$ \\
\hline 1.64 & $1.48 \mathrm{E}-07$ & $1.52 \mathrm{E}-07$ & 7.10 & $3.46 \mathrm{E}-08$ & $3.90 \mathrm{E}-08$ \\
\hline $\begin{array}{l}1.72 \\
1.80\end{array}$ & $\begin{array}{l}1.50 \mathrm{E}-07 \\
1.61 \mathrm{E}-07\end{array}$ & $\begin{array}{l}1.54 \mathrm{E}-07 \\
1.65 \mathrm{E}-07\end{array}$ & & & $\begin{array}{r}4.02 \mathrm{E}-08 \\
4.1 . \mathrm{E}-08\end{array}$ \\
\hline 1.88 & $1.78 \mathrm{E}-07$ & $1.82 \mathrm{E}-07$ & 7.70 & $3.66 \mathrm{E}-$ & $3.99 \mathrm{E}-08$ \\
\hline 1.96 & $1.90 \mathrm{E}-07$ & $1.94 \mathrm{E}-07$ & 7.90 & $3.10 \mathrm{E}-$ & $3.39 \mathrm{E}-08$ \\
\hline 2.04 & $1.94 \mathrm{E}-07$ & $1.97 \mathrm{E}-07$ & 8.10 & $2.28 \mathrm{E}-08$ & $2.54 \mathrm{E}-08$ \\
\hline 2.12 & $1.91 \mathrm{E}-07$ & $1.95 \mathrm{E}-07$ & 8.30 & $1.46 \mathrm{E}-08$ & $1.69 \mathrm{E}-08$ \\
\hline 2.20 & $1.84 \mathrm{E}-07$ & $1.87 \mathrm{E}-07$ & 8.50 & $8.38 \mathrm{E}-09$ & $1.03 \mathrm{E}-08$ \\
\hline 2.28 & $1.69 \mathrm{E}-07$ & $1.72 \mathrm{E}-07$ & 8.73 & $4.22 \mathrm{E}-09$ & $5.92 \mathrm{E}-09$ \\
\hline 2.36 & $1.47 \mathrm{E}-07$ & $1.50 \mathrm{E}-07$ & 8.98 & $2.69 \mathrm{E}-09$ & $4.10 \mathrm{E}-09$ \\
\hline 2.45 & $1.17 \mathrm{E}-07$ & $1.20 \mathrm{E}-07$ & 9.23 & $2.72 \mathrm{E}-09$ & $3.88 \mathrm{E}-09$ \\
\hline 2.55 & $8.92 \mathrm{E}-08$ & $9.17 \mathrm{E}-08$ & 9.48 & $2.82 \mathrm{E}-09$ & $4.03 \mathrm{E}-09$ \\
\hline 2.65 & $7.29 \mathrm{E}-08$ & $7.54 \mathrm{E}-08$ & 9.73 & $2.43 \mathrm{E}-09$ & $3.83 \mathrm{E}-09$ \\
\hline 2.75 & $6.69 \mathrm{E}-08$ & $6.94 \mathrm{E}-08$ & 9.98 & $1.66 \mathrm{E}-09$ & $3.20 \mathrm{E}-09$ \\
\hline 2.85 & $6.50 \mathrm{E}-08$ & $6.75 \mathrm{E}-08$ & 10.30 & $6.55 \mathrm{E}-10$ & $2.23 \mathrm{E}-09$ \\
\hline 2.95 & $6.21 \mathrm{E}-08$ & $6.46 \mathrm{E}-08$ & 10.70 & $-6.80 E-11$ & $1.12 \mathrm{E}-09$ \\
\hline 3.05 & $5.69 E-08$ & $5.94 \mathrm{E}-08$ & 11.10 & $-3.11 \mathrm{E}-10$ & $4.65 \mathrm{E}-10$ \\
\hline 3.15 & $5.11 \mathrm{E}-08$ & $5.38 E-08$ & 11.50 & $-4.92 \mathrm{E}-10$ & $3.88 \mathrm{E}-10$ \\
\hline 3.25 & $4.76 E-08$ & $5.03 \mathrm{E}-08$ & 11.90 & $-5.34 \mathrm{E}-10$ & $4.31 \mathrm{E}-10$ \\
\hline 3.35 & $4.75 \mathrm{E}-08$ & $5.02 \mathrm{E}-08$ & 12.30 & $-4.61 \mathrm{E}-10$ & $4.01 \mathrm{E}-10$ \\
\hline 3.45 & $4.98 \mathrm{E}-08$ & $5.25 \mathrm{E}-08$ & 12.70 & $-4.01 \mathrm{E}-10$ & $3.57 \mathrm{E}-10$ \\
\hline 3.55 & $5.24 \mathrm{E}-08$ & $5.53 \mathrm{E}-08$ & 13.10 & $-3.54 \mathrm{E}-10$ & $3.09 \mathrm{E}-10$ \\
\hline 3.66 & $5.37 \mathrm{E}-08$ & $5.68 E-08$ & 13.50 & $-2.89 \mathrm{E}-10$ & $2.61 \mathrm{E}-10$ \\
\hline 3.79 & $5.20 \mathrm{E}-08$ & $5.52 \mathrm{E}-08$ & 13.90 & $-2.15 \mathrm{E}-10$ & 2. $28 \mathrm{E}-10$ \\
\hline
\end{tabular}




$$
\begin{gathered}
\text { Table A.8 } \\
\text { Neutron Data } \\
\text { Detector } 8 \text { in Fig. } 4 \\
\text { Detector Coordinates }(\mathrm{cm}): \quad x=0.0, y=0.0, z^{\prime}=151.0
\end{gathered}
$$

\begin{tabular}{|c|c|c|c|}
\hline $\begin{array}{l}\text { Energy } \\
(\mathrm{MeV})\end{array}$ & $\begin{array}{l}\text { Fluence }\left(\mathrm{MeV}^{-1} \mathrm{~cm}^{-2}\right) \\
\text { Confidence Interval }\end{array}$ & $\begin{array}{c}\text { Energy } \\
(\mathrm{MeV})\end{array}$ & Fluence $\left(\mathrm{MeV}^{-1} \mathrm{~cm}^{-2}\right)$ \\
\hline
\end{tabular}

\begin{tabular}{|c|c|c|c|c|c|}
\hline 0.91 & 1.13E-06 & $1.14 E-06$ & 5.94 & $5.65 E-08$ & $6.03 E-08$ \\
\hline 1.01 & 1.11E-06 & 1.12E-06 & 6.25 & 4.61E-08 & 5.05E-08 \\
\hline 1.11 & 9.52E-07 & $9.66 \mathrm{E}-07$ & 6.55 & 4.75E-08 & $5.21 \mathrm{E}-08$ \\
\hline 1.21 & 8.48E-07 & 8.61E-06 & 6.83 & $5.50 \mathrm{E}-08$ & $5.90 \mathrm{E}-08$ \\
\hline 1.31 & 8.67E-07 & 8.77E-07 & 7.23 & 5.79E-08 & 6.17E-08 \\
\hline 1.41 & $9.40 \mathrm{E}-07$ & 9.52E-07 & 7.73 & $3.90 \mathrm{E}-08$ & 4.33E-08 \\
\hline 1.51 & 9.49E-07 & 9.61E-07 & 8.25 & 6.33E-09 & $1.04 \mathrm{E}-08$ \\
\hline 1.61 & 8.58E-07 & 8.67E-07 & 8.88 & 1.87E-09 & $5.59 \mathrm{E}-08$ \\
\hline 1.71 & $7.03 E-07$ & $7.10 \mathrm{E}-07$ & 9.24 & 9.51E-09 & 1.35E-08 \\
\hline 1.81 & $5.48 \mathrm{E}-07$ & $5.56 \mathrm{E}-07$ & 9.72 & $1.15 E-08$ & $1.52 \mathrm{E}-08$ \\
\hline 1.94 & 4.28E-07 & 4.37E-07 & 10.23 & $1.69 \mathrm{E}-08$ & 2.07E-08 \\
\hline 2.11 & $3.96 \mathrm{E}-07$ & 4.02E-07 & 10.75 & $2.86 \mathrm{E}-08$ & 3.29E-07 \\
\hline 2.31 & $3.65 E-07$ & 3.73E-07 & 11.26 & $3.26 \mathrm{E}-08$ & $3.69 E-08$ \\
\hline 2.50 & 2.89E-07 & 2.94E-07 & 11.76 & $2.73 E-08$ & $3.15 \mathrm{E}-08$ \\
\hline 2.69 & $2.39 \mathrm{E}-07$ & $2.44 \mathrm{E}-07$ & 12.40 & $7.81 \mathrm{E}-08$ & 8.25E-08 \\
\hline 2.90 & $2.68 \mathrm{E}-07$ & 2.74E-07 & 13.20 & 4.14E-07 & 4.18E-07 \\
\hline 3.10 & 2.89E-07 & $2.96 \mathrm{E}-07$ & 14.03 & $9.44 E-07$ & 9.48E-07 \\
\hline 3.30 & 2.44E-07 & 2.49E-07 & 14.86 & $1.16 \mathrm{E}-06$ & $1.16 \mathrm{E}-06$ \\
\hline 3.51 & 1.78E-07 & $1.84 \mathrm{E}-07$ & 15.62 & 9.49E-07 & 9.52E-07 \\
\hline 3.71 & $1.44 \mathrm{E}-07$ & 1.51E-07 & 16.45 & 5.51E-07 & 5.53E-07 \\
\hline 3.90 & $1.36 \mathrm{E}-07$ & $1.41 \mathrm{E}-07$ & 17.44 & 2.10E-07 & 2.11E-07 \\
\hline 4.15 & $1.25 \mathrm{E}-07$ & 1.30E-07 & 18.51 & $5.36 \mathrm{E}-08$ & $5.39 \mathrm{E}-08$ \\
\hline 4.46 & 1.04E-07 & $1.08 E-07$ & 19.58 & 9.86E-09 & 9.99E-09 \\
\hline 4.75 & 8.95E-08 & 9.33E-08 & 20.53 & $6.76 \mathrm{E}-11$ & $1.36 \mathrm{E}-10$ \\
\hline 5.06 & 7.64E-08 & 8.08E-08 & 21.43 & $-2.45 E-09$ & $-2.40 \mathrm{E}-09$ \\
\hline 5.36 & 6.59E-08 & $7.08 \mathrm{E}-08$ & 22.40 & $-2.90 \mathrm{E}-09$ & $-2.87 E-09$ \\
\hline 5.64 & 6.34E-08 & 6.75E-08 & 23.43 & $-2.40 \mathrm{E}-09$ & $-2.38 \mathrm{E}-09$ \\
\hline
\end{tabular}


Table A.8 (Cont'd)

Gamma-Ray Data

Detector 8 in Fig. 4

Detector Coordinates $(\mathrm{cm}): x=0.0, y=0.0, z^{\prime}=151.0$

\begin{tabular}{|c|c|c|c|c|c|}
\hline $\begin{array}{l}\text { Energy } \\
(\mathrm{MeV})\end{array}$ & \multicolumn{2}{|c|}{$\begin{array}{l}\left.\text { Fluence (MeV-1 } \mathrm{cm}^{-2}\right) \\
\text { Confidence Interval }\end{array}$} & $\begin{array}{l}\text { Energy } \\
(\mathrm{MeV})\end{array}$ & \multicolumn{2}{|c|}{$\begin{array}{c}\text { Fuence }\left(\mathrm{MeV}^{-1} \mathrm{~cm}^{-2}\right) \\
\text { Confidence Interval }\end{array}$} \\
\hline 0.72 & $1.27 \mathrm{E}-06$ & $2.11 E-06$ & 3.93 & 1.67E-07 & $1.79 \mathrm{E}-07$ \\
\hline 0.76 & $2.36 \mathrm{E}-06$ & $2.70 \mathrm{E}-06$ & 4.06 & 1.39E-07 & $1.52 \mathrm{E}-07$ \\
\hline 0.80 & 3.07E-06 & $3.16 \mathrm{E}-06$ & 4.19 & $1.31 \mathrm{E}-07$ & $1.45 \mathrm{E}-07$ \\
\hline 0.84 & $3.24 \mathrm{E}-06$ & 3.27E-06 & 4.31 & $1.48 \mathrm{E}-07$ & $1.63 \mathrm{E}-07$ \\
\hline 0.88 & 2.95E-06 & $2.98 \mathrm{E}-06$ & 4.44 & $1.85 E-07$ & 2.04E-07 \\
\hline 0.92 & 2.44E-06 & 2.47E-06 & 4.57 & $2.27 \mathrm{E}-07$ & $2.47 \mathrm{E}-07$ \\
\hline 0.96 & $1.94 \mathrm{E}-06$ & 1.97E-06 & 4.70 & $2.48 \mathrm{E}-07$ & $2.68 \mathrm{E}-07$ \\
\hline 1.00 & $1.58 \mathrm{E}-06$ & $1.00 \mathrm{E}-06$ & 4.83 & $2.29 \mathrm{E}-07$ & $2.50 \mathrm{E}-07$ \\
\hline 1.04 & $1.36 \mathrm{E}-06$ & $1.38 E-06$ & 4.99 & $1.63 E-07$ & $1.87 \mathrm{E}-07$ \\
\hline 1.08 & $1.29 \mathrm{E}-06$ & $1.31 \mathrm{E}-06$ & 5.15 & 8.98E-08 & $1.16 \mathrm{E}-07$ \\
\hline 1.13 & $1.33 E-06$ & $1.35 E-06$ & 5.32 & $7.20 E-08$ & $1.00 \mathrm{E}-07$ \\
\hline 1.17 & $1.44 E-06$ & $1.46 \mathrm{E}-06$ & 5.50 & $9.76 \mathrm{E}-08$ & $1.27 \mathrm{E}-07$ \\
\hline 1.22 & $1.52 \mathrm{E}-06$ & $1.54 E-06$ & 5.67 & $1.23 \mathrm{E}-07$ & $1.55 \mathrm{E}-07$ \\
\hline 1.28 & $1.50 \mathrm{E}-06$ & $1.52 \mathrm{E}-06$ & 5.83 & $1.24 \mathrm{E}-07$ & $1.54 \mathrm{E}-07$ \\
\hline 1.33 & $1.38 \mathrm{E}-06$ & $1.40 \mathrm{E}-06$ & 6.00 & 1.12E-07 & 1.39E-07 \\
\hline 1.38 & $1.22 \mathrm{E}-06$ & $1.24 \mathrm{E}-06$ & 6.18 & $1.04 \mathrm{E}-07$ & 1.32E-07 \\
\hline 1.42 & 1.04E-06 & $1.06 \mathrm{E}-06$ & 6.35 & $1.06 \mathrm{E}-07$ & $1.33 E-07$ \\
\hline 1.47 & $8.74 E-07$ & 8.89E-07 & 6.51 & $1.10 \mathrm{E}-07$ & 1.33E-07 \\
\hline 1.53 & $7.40 \mathrm{E}-07$ & $7.54 E-07$ & 6.70 & 1.02E-07 & 1.22E-07 \\
\hline 1.58 & $6.55 \mathrm{E}-07$ & 6.69E-07 & 6.90 & 8.37E-08 & $1.02 E-07$ \\
\hline 1.64 & $6.14 E-07$ & $6.27 E-07$ & 7.10 & $6.96 \mathrm{E}-08$ & $8.70 E-08$ \\
\hline 1.72 & $6.22 E-07$ & $6.36 \mathrm{E}-07$ & 7.30 & $7.16 \mathrm{E}-08$ & 8.85E-08 \\
\hline 1.80 & 6.33E-07 & $6.46 \mathrm{E}-07$ & 7.50 & $8.40 E-08$ & $1.00 \mathrm{E}-07$ \\
\hline 1.88 & $6.12 \mathrm{E}-07$ & $6.26 E-07$ & 7.70 & 9.09E-08 & $1.06 \mathrm{E}-07$ \\
\hline 1.96 & $5.64 \mathrm{E}-07$ & $5.78 E-07$ & 7.90 & 8.38E-08 & $9.82 \mathrm{E}-08$ \\
\hline 2.04 & $5.18 \mathrm{E}-07$ & $5.30 \mathrm{E}-07$ & 8.10 & $6.45 E-08$ & $7.82 \mathrm{E}-08$ \\
\hline 2.12 & 4.91E-07 & $5.02 E-07$ & 8.30 & 4.31E-08 & $5.54 \mathrm{E}-08$ \\
\hline 2.20 & 4.69E-07 & $4.80 \mathrm{E}-07$ & 8.50 & 2.88E-08 & 4.05E-08 \\
\hline 2.28 & 4.32E-07 & $4.43 E-07$ & 8.73 & $2.44 E-08$ & $3.51 E-08$ \\
\hline 2.36 & $3.84 E-07$ & $3.95 \mathrm{E}-07$ & 8.98 & $2.97 \mathrm{E}-08$ & $3.94 \mathrm{E}-08$ \\
\hline 2.45 & $3.47 E-07$ & $3.58 \mathrm{E}-07$ & 9.23 & $3.61 E-08$ & $4.50 \mathrm{E}-08$ \\
\hline 2.55 & $3.31 \mathrm{E}-07$ & $3.41 E-07$ & 9.48 & $3.60 \mathrm{E}-08$ & $4.39 \mathrm{E}-08$ \\
\hline 2.65 & $3.18 \mathrm{E}-07$ & $3.28 \mathrm{E}-07$ & 9.73 & $2.86 \mathrm{E}-08$ & $3.55 E-08$ \\
\hline 2.75 & $2.98 \mathrm{E}-07$ & $3.08 \mathrm{E}-07$ & 9.98 & $1.65 E-08$ & $2.28 \mathrm{E}-08$ \\
\hline 2.85 & 2.76E-07 & $2.80 \mathrm{E}-07$ & 10.30 & $9.97 E-10$ & 6.33E-09 \\
\hline 2.95 & 2.59E-07 & 2.68E-07 & 10.70 & $-1.21 \mathrm{E}-08$ & $-6.99 \mathrm{E}-09$ \\
\hline 3.05 & $2.46 \mathrm{E}-07$ & $2.56 E-07$ & 11.10 & $-1.84 E-08$ & $-1.23 E-08$ \\
\hline 3.15 & 2.39E-07 & 2.49E-07 & 11.50 & $-1.97 \mathrm{E}-08$ & $-1.30 \mathrm{E}-08$ \\
\hline 3.25 & 2.35E-07 & $2.45 E-07$ & 11.90 & $-1.72 E-08$ & $-1.07 E-08$ \\
\hline 3.35 & 2.32E-07 & 2.42E-07 & 12.30 & $-1.08 \mathrm{E}-08$ & $-5.72 E-09$ \\
\hline 3.45 & $2.31 E-07$ & $2.41 E-07$ & 13.70 & $-1.89 \mathrm{E}-09$ & 1.15E-09 \\
\hline 3.55 & $2.30 \mathrm{E}-07$ & $2.40 E_{-07}$ & 13.10 & $6.39 \mathrm{E}-09$ & 8.23E-09 \\
\hline 3.66 & 2.22E-07 & 2.34E-07 & 13.50 & $1.09 E-08$ & $1.40 \mathrm{E}-08$ \\
\hline 3.79 & 2.00E-07 & $2.12 \mathrm{E}-07$ & 13.90 & 1.17E-08 & $1.55 \mathrm{E}-08$ \\
\hline
\end{tabular}


Table A.9

Neutron bata

Detector 9 in Fig. 4

Detector Coordinates $(\mathrm{cm}): x=59.0, y=0.0, z^{\prime}=151.0$

\begin{tabular}{|c|c|c|c|c|c|}
\hline $\begin{array}{l}\text { Energy } \\
(\mathrm{MeV})\end{array}$ & \multicolumn{2}{|c|}{$\underline{F} \underline{1}$ 므므 $\underline{c} e-(\underline{M} \underline{\mathrm{V}}-1$} & $\begin{array}{l}\text { Energy } \\
(\mathrm{MeV})\end{array}$ & \multicolumn{2}{|c|}{ 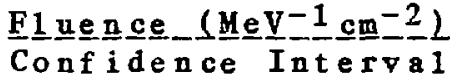 } \\
\hline 0.91 & $2.07 \mathrm{E}-07$ & $2.09 \mathrm{E}-07$ & 5.94 & $6.15 \mathrm{E}-09$ & $6.51 \mathrm{E}-0.9$ \\
\hline 1.01 & $1.95 \mathrm{E}-07$ & $1.96 \mathrm{E}-07$ & 6.25 & $5.59 \mathrm{E}-09$ & $5.99 \mathrm{E}-09$ \\
\hline 1.11 & $1.77 \mathrm{E}-07$ & $1.79 E-07$ & 6.55 & $5.37 \mathrm{E}-09$ & $5.80 \mathrm{E}-09$ \\
\hline 1.21 & $1.59 \mathrm{E}-07$ & $1.60 \mathrm{E}-07$ & 6.83 & $5.24 \mathrm{E}-09$ & $5.60 \mathrm{E}-09$ \\
\hline 1.31 & $1.41 \mathrm{E}-07$ & $1.43 \mathrm{E}-07$ & 7.23 & $4.64 \mathrm{E}-09$ & $4.97 E-09$ \\
\hline 1.41 & $1.26 \mathrm{E}-07$ & $1.27 \mathrm{E}-07$ & 7.73 & $3.52 \mathrm{E}-09$ & $3.90 \mathrm{E}-09$ \\
\hline 1.51 & $1.14 \mathrm{E}-07$ & $1.15 \mathrm{E}-07$ & 8.25 & $2.55 \mathrm{E}-09$ & $2.90 \mathrm{E}-09$ \\
\hline 1.61 & $1.02 \mathrm{E}-07$ & $1.03 \mathrm{E}-07$ & 8.77 & $2.38 \mathrm{E}-09$ & $2.67 \mathrm{E}-09$ \\
\hline 1.71 & $9.07 \mathrm{E}-08$ & $9.17 \mathrm{E}-08$ & 9.24 & $2.54 \mathrm{E}-09$ & $2.84 \mathrm{E}-09$ \\
\hline 1.81 & $8.02 \mathrm{E}-08$ & $8.15 \mathrm{E}-08$ & 9.72 & $2.58 \mathrm{E}-0.9$ & $2.85 \mathrm{E}-09$ \\
\hline 1.94 & $7.08 \mathrm{E}-08$ & $7.20 \mathrm{E}-08$ & 10.23 & $2.57 \mathrm{E}-09$ & $2.85 \mathrm{E}-09$ \\
\hline 2.11 & $6.40 \mathrm{E}-08$ & $6.48 \mathrm{E}-08$ & 10.75 & $2.50 \mathrm{E}-09$ & $2.79 \mathrm{E}-09$ \\
\hline 2.31 & $5.56 \mathrm{E}-08$ & $5.66 \mathrm{E}-08$ & 11.26 & $2.33 \mathrm{E}-09$ & $2.62 \mathrm{E}-09$ \\
\hline 2.50 & $4.60 E-08$ & $4.67 E-08$ & 11.76 & $2.49 \mathrm{E}-09$ & $2.77 \mathrm{E}-09$ \\
\hline 2.69 & $3.79 E-08$ & $3.85 \mathrm{E}-08$ & 12.40 & $4.03 \mathrm{E}-09$ & $4.31 \mathrm{E}-09$ \\
\hline 2.90 & $3.17 \mathrm{E}-08$ & $3.23 \mathrm{E}-08$ & 13.20 & $8.71 \mathrm{E}-09$ & $8.95 \mathrm{E}-09$ \\
\hline 3.10 & $2.60 E-08$ & $2.68 \mathrm{E}-08$ & 14.03 & $1.40 \mathrm{E}-0 \mathrm{~B}$ & $1.42 \mathrm{E}-0 \mathrm{~B}$ \\
\hline 3.30 & $2.14 \mathrm{E}-08$ & $2.20 \mathrm{E}-08$ & 14.86 & $1.43 \mathrm{E}-08$ & $1.44 \mathrm{E}-08$ \\
\hline 3.51 & $1.80 \mathrm{E}-08$ & $1.87 E-08$ & 15.62 & $1.01 E-08$ & $1.02 \mathrm{E}-08$ \\
\hline 3.71 & $1.59 \mathrm{E}-08$ & $1.67 \mathrm{E}-08$ & 16.45 & $5.00 \mathrm{E}-09$ & $5.09 \mathrm{E}-09$ \\
\hline $\begin{array}{l}3.90 \\
4.15\end{array}$ & $\begin{array}{l}1.46 \mathrm{E}-08 \\
1.27 \mathrm{E}-08\end{array}$ & $\begin{array}{l}1.51 \mathrm{E}-08 \\
1.33 \mathrm{E}-08\end{array}$ & $\begin{array}{l}17.44 \\
18.51\end{array}$ & $\begin{array}{l}1.61 \mathrm{E}-09 \\
3.54 \mathrm{E}-10\end{array}$ & $\begin{array}{l}1.65 \mathrm{E}-09 \\
3.75 \mathrm{E}-10\end{array}$ \\
\hline 4.46 & $1.10 \mathrm{E}-08$ & $1.13 \mathrm{E}-08$ & 19.58 & $4.83 \mathrm{E}-11$ & $6.64 \mathrm{E}-11$ \\
\hline 4.75 & $9.66 \mathrm{E}-09$ & $1.01 \mathrm{E}-08$ & 20.53 & $-1.77 \mathrm{E}-11$ & $-1.67 \mathrm{E}-12$ \\
\hline 5.06 & $8.40 E-09$ & $8.84 E-09$ & 21.43 & $-3.62 \mathrm{E}-11$ & $-2.31 \mathrm{E}-11$ \\
\hline 5.36 & $7.31 \mathrm{E}-09$ & $7.79 \mathrm{E}-09$ & 22.40 & $-4.29 \mathrm{E}-11$ & $-2.87 E-11$ \\
\hline 5.64 & $6.70 \mathrm{E}-09$ & $7.12 \mathrm{E}-09$ & 23.43 & $-3.85 E-11$ & $-2.28 \mathrm{E}-11$ \\
\hline
\end{tabular}


Table A. 9 (Cont'd)

Gamma-Ray Data

Detector 9 in Fig. 4

Detector Coordinates $(\mathrm{cm}): x=59.0, y=0.0, z^{\prime}=151.0$

\begin{tabular}{|c|c|c|c|c|c|}
\hline \multirow{2}{*}{$\begin{array}{l}\text { Energy } \\
(M e V)\end{array}$} & \multicolumn{2}{|c|}{ 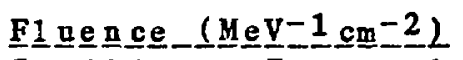 } & \multirow{2}{*}{$\begin{array}{l}\text { Energy } \\
(\mathrm{MeV})\end{array}$} & \multicolumn{2}{|c|}{ 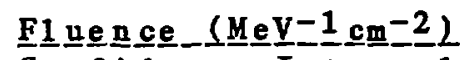 } \\
\hline & Confidence & Interva 1 & & & Interval \\
\hline 0.72 & $2.01 \mathrm{E}-07$ & $3.36 \mathrm{E}-07$ & 3.93 & $3.44 \mathrm{E}-08$ & $3.73 \mathrm{E}-08$ \\
\hline 0.76 & $3.22 \mathrm{E}-07$ & $3.77 \mathrm{E}-07$ & 4.06 & $3.11 \mathrm{E}-08$ & $3.42 \mathrm{E}-08$ \\
\hline $\begin{array}{l}0.80 \\
0.84\end{array}$ & $\begin{array}{l}3.83 \mathrm{E}-07 \\
3.85 \mathrm{E}-07\end{array}$ & $\begin{array}{l}3.99 \mathrm{E}-07 \\
3.91 \mathrm{E}-07\end{array}$ & $\begin{array}{l}4.19 \\
4.31\end{array}$ & $\begin{array}{l}2.95 \mathrm{E}-08 \\
2.97 \mathrm{E}-08\end{array}$ & $\begin{array}{l}3.28 \mathrm{E}-08 \\
3.35 \mathrm{E}-08\end{array}$ \\
\hline 0.88 & $3.48 \mathrm{E}-07$ & $3.53 \mathrm{E}-07$ & 4.44 & $3.14 \mathrm{E}-08$ & $3.59 \mathrm{E}-08$ \\
\hline 0.92 & $2.99 \mathrm{E}-07$ & $3.04 \mathrm{E}-07$ & 4.57 & $3.37 \mathrm{E}-08$ & $3.85 E-08$ \\
\hline 0.96 & $2.54 \mathrm{E}-07$ & $2.59 \mathrm{E}-07$ & 4.70 & $3.44 \mathrm{E}-08$ & $3.93 \mathrm{E}-08$ \\
\hline 1.00 & $2.21 \mathrm{E}-07$ & $2.25 \mathrm{E}-07$ & 4.83 & $3.22 \mathrm{E}-08$ & $3.74 \mathrm{E}-08$ \\
\hline 1.04 & $2.00 \mathrm{E}-07$ & $2.04 \mathrm{E}-07$ & 4.99 & $2.64 \mathrm{E}-08$ & $3.26 \mathrm{E}-08$ \\
\hline 1.08 & $1.91 \mathrm{E}-07$ & $1.94 \mathrm{E}-07$ & 5.15 & $1.93 \mathrm{E}-08$ & $2.59 \mathrm{E}-08$ \\
\hline 1.13 & $1.90 \mathrm{E}-07$ & $1.94 \mathrm{E}-07$ & 5.32 & $1.57 \mathrm{E}-08$ & $2.24 \mathrm{E}-08$ \\
\hline 1.17 & $1.93 \mathrm{E}-07$ & $1.96 \mathrm{E}-07$ & 5.50 & $1.77 \mathrm{E}-08$ & $2.51 \mathrm{E}-08$ \\
\hline 1.22 & $1.93 \mathrm{E}-07$ & $1.97 \mathrm{E}-07$ & 5.67 & $2.56 \mathrm{E}-08$ & $3.33 \mathrm{E}-08$ \\
\hline 1.28 & $1.88 \mathrm{E}-07$ & $1.93 \mathrm{E}-07$ & 5.83 & $3.54 \mathrm{E}-08$ & $4.27 \mathrm{E}-08$ \\
\hline 1.33 & $1.77 E-07$ & $1.81 \mathrm{E}-07$ & 6.00 & $4.26 \mathrm{E}-08$ & $4.95 \mathrm{E}-08$ \\
\hline 1.38 & $1.63 \mathrm{E}-07$ & $1.66 \mathrm{E}-07$ & 6.18 & $4.45 \mathrm{E}-08$ & $5.12 E-08$ \\
\hline 1.42 & $1.49 \mathrm{E}-07$ & $1.53 \mathrm{E}-07$ & 6.35 & $4.09 E-08$ & $4.71 E-08$ \\
\hline 1.47 & $1.38 \mathrm{E}-07$ & $1.42 \mathrm{E}-07$ & 6.51 & $3.47 E-08$ & $4.01 \mathrm{E}-08$ \\
\hline 1.53 & $1.29 \mathrm{E}-07$ & 1.32 E- 07 & 6.70 & $2.86 \mathrm{E}-08$ & $3.32 \mathrm{E}-08$ \\
\hline 1.58 & $1.22 \mathrm{E}-07$ & $1.25 \mathrm{E}-07$ & 6.90 & $2.60 \mathrm{E}-08$ & $3.00 \mathrm{E}-08$ \\
\hline $\begin{array}{l}1.64 \\
1.72\end{array}$ & $\begin{array}{l}1.20 \mathrm{E}-07 \\
1.26 \mathrm{E}-07\end{array}$ & $\begin{array}{l}1.23 \mathrm{E}-07 \\
1.30 \mathrm{E}-07\end{array}$ & $\begin{array}{l}7.10 \\
7.30\end{array}$ & $\begin{array}{l}2.70 \mathrm{E}-08 \\
2.91 \mathrm{E}-08\end{array}$ & $\begin{array}{l}3.09 \mathrm{E}-08 \\
3.26 \mathrm{E}-08\end{array}$ \\
\hline 1.80 & $1.36 \mathrm{E}-07$ & & & & \\
\hline 1.88 & $1.42 \mathrm{E}-07$ & $1.46 \mathrm{E}-07$ & 7.70 & $2.49 \mathrm{E}-08$ & $2.7 .8 \mathrm{E}-08$ \\
\hline 1.96 & $1.46 \mathrm{E}-07$ & $1.49 \mathrm{E}-07$ & 7.90 & $1.84 \mathrm{E}-08$ & $2.10 \mathrm{E}-08$ \\
\hline 2.04 & $1.45 \mathrm{E}-07$ & $1.48 \mathrm{E}-07$ & 8.10 & $1.16 \mathrm{E}-08$ & $1.40 E-08$ \\
\hline 2.12 & $1.41 \mathrm{E}-07$ & $1.44 \mathrm{E}-07$ & 8.30 & $6.64 \mathrm{E}-09$ & $8.65 \mathrm{E}-09$ \\
\hline 2.20 & $1.35 E-07$ & $1.38 \mathrm{E}-07$ & 8.50 & $4.00 \mathrm{E}-09$ & $5.73 E-09$ \\
\hline 2.28 & $1.23 E-07$ & $1.25 \mathrm{E}-07$ & 8.73 & $3.01 \mathrm{E}-09$ & $4.52 E-09$ \\
\hline 2.36 & $1.04 \mathrm{E}-07$ & $1.07 \mathrm{E}-07$ & 8.98 & $2.83 \mathrm{E}-09$ & $4.15 \mathrm{E}-09$ \\
\hline 2.45 & $8.13 \mathrm{E}-08$ & $8.38 \mathrm{E}-08$ & 9.23 & $2.48 \mathrm{E}-09$ & $3.59 \mathrm{E}-09$ \\
\hline 2.55 & $6.42 \mathrm{E}-08$ & $6.65 \mathrm{E}-08$ & 9.48 & $1.76 E-09$ & $2.72 \mathrm{E}-09$ \\
\hline 2.65 & $5.72 E-08$ & $5.95 \mathrm{E}-08$ & 9.73 & $9.18 \mathrm{E}-10$ & $1.75 \mathrm{E}-09$ \\
\hline 2.75 & $5.46 \mathrm{E}-08$ & $5.69 \mathrm{E}-08$ & 9.98 & $2.36 \mathrm{E}-10$ & $1.03 \mathrm{E}-09$ \\
\hline 2.85 & $5.21 E-08$ & $5.44 \mathrm{E}-08$ & 10.30 & $-1.81 E-10$ & $6.23 E-10$ \\
\hline 2.95 & $4.90 \mathrm{E}-08$ & $5.12 \mathrm{E}-08$ & & $-1.73 E-10$ & $6.90 \mathrm{E}-10$ \\
\hline 3.05 & $4.53 \mathrm{E}-08$ & $4.75 \mathrm{E}-08$ & 11.10 & $-1.60 E-11$ & $8.23 \mathrm{E}-10$ \\
\hline 3.15 & $4.19 \mathrm{E}-08$ & $4.44 \mathrm{E}-08$ & 11.50 & $6.31 \mathrm{E}-11$ & $6.90 \mathrm{E}-10$ \\
\hline 3.25 & $4.05 \mathrm{E}-08$ & $4.30 \mathrm{E}-08$ & 11.90 & $2.51 \mathrm{E}-11$ & $4.56 \mathrm{E}-10$ \\
\hline 3.35 & $4.14 \mathrm{E}-08$ & $4.39 \mathrm{E}-08$ & 12.30 & $-9.61 \mathrm{E}-11$ & $3.69 \mathrm{E}-10$ \\
\hline 3.45 & $4.34 \mathrm{E}-08$ & $4.58 \mathrm{E}-08$ & 12.70 & $-1.10 \mathrm{E}-10$ & 3. $94 \mathrm{E}-10$ \\
\hline 3.55 & $4.45 \mathrm{E}-08$ & $4.71 E-08$ & 13.10 & $-9.10 \mathrm{E}-11$ & $3.67 \mathrm{E}-10$ \\
\hline 3.66 & $4.32 \mathrm{E}-08$ & $4.60 E-08$ & 13.50 & $-7.65 \mathrm{E}-11$ & $3.15 \mathrm{E}-10$ \\
\hline 3.79 & $3.90 \mathrm{E}-08$ & $4.19 \mathrm{E}-08$ & 13.90 & $-6.41 \mathrm{E}-11$ & $2.43 \mathrm{E}-10$ \\
\hline
\end{tabular}


Table A.10

Neutron Data

Detector 10 in Fig. 4

Detector Coordinates $(\mathrm{cm}): x=79.0, y=0.0, z^{\prime} 151.0$

\begin{tabular}{|c|c|c|c|c|c|}
\hline $\begin{array}{l}\text { Energy } \\
(\mathrm{MeV})\end{array}$ & 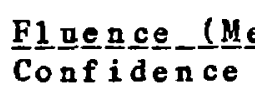 & $=\frac{v^{-1}}{I n t} \frac{m^{-2}}{r}$ - 1 & $\begin{array}{l}\text { Energy } \\
(\mathrm{HeV})\end{array}$ & Fon & $=\frac{V^{-1}}{I n t e m}-\frac{2}{r}=\frac{2}{a}$ \\
\hline $\begin{array}{l}0.91 \\
1.01 \\
1.11 \\
1.21 \\
1.31 \\
1.41 \\
1.51 \\
1.61 \\
1.71 \\
1.81 \\
1.94 \\
2.11 \\
2.31 \\
2.50 \\
2.69 \\
2.90 \\
3.10 \\
3.30 \\
3.51 \\
3.71 \\
3.90 \\
4.15 \\
4.46 \\
4.75 \\
5.06 \\
5.36 \\
5.64\end{array}$ & $\begin{array}{l}1.27 \mathrm{E}-07 \\
1.19 \mathrm{E}-07 \\
1.01 \mathrm{E}-07 \\
9.50 \mathrm{E}-08 \\
8.48 \mathrm{E}-08 \\
7.65 \mathrm{E}-08 \\
6.99 \mathrm{E}-08 \\
6.32 \mathrm{E}-08 \\
5.64 \mathrm{E}-08 \\
5.02 \mathrm{E}-08 \\
4.45 \mathrm{E}-08 \\
4.04 \mathrm{E}-08 \\
3.56 \mathrm{E}-08 \\
3.00 \mathrm{E}-08 \\
2.47 \mathrm{E}-08 \\
2.00 \mathrm{E}-08 \\
1.60 \mathrm{E}-08 \\
1.30 \mathrm{E}-08 \\
1.08 \mathrm{E}-08 \\
9.38 \mathrm{E}-09 \\
8.73 \mathrm{E}-09 \\
7.96 \mathrm{E}-09 \\
6.83 \mathrm{E}-09 \\
5.83 \mathrm{E}-09 \\
5.17 \mathrm{E}-09 \\
4.69 \mathrm{E}-09 \\
4.30 \mathrm{E}-09\end{array}$ & $\begin{array}{l}1.28 \mathrm{E}-07 \\
1.19 \mathrm{E}-07 \\
1.07 \mathrm{E}-07 \\
9.57 \mathrm{E}-08 \\
8.55 \mathrm{E}-08 \\
7.72 \mathrm{E}-08 \\
7.07 \mathrm{E}-08 \\
6.38 \mathrm{E}-08 \\
5.69 \mathrm{E}-08 \\
5.09 \mathrm{E}-08 \\
4.51 \mathrm{E}-08 \\
4.08 \mathrm{E}-08 \\
3.61 \mathrm{E}-08 \\
3.03 \mathrm{E}-0.8 \\
2.50 \mathrm{E}-08 \\
2.04 \mathrm{E}-08 \\
1.64 \mathrm{E}-08 \\
1.33 \mathrm{E}-08 \\
1.11 \mathrm{E}-08 \\
9.79 \mathrm{E}-09 \\
9.01 \mathrm{E}-09 \\
8.27 \mathrm{E}-09 \\
7.01 \mathrm{E}-09 \\
6.03 \mathrm{E}-09 \\
5.39 \mathrm{E}-09 \\
4.93 \mathrm{E}-09 \\
4.51 \mathrm{E}-09\end{array}$ & $\begin{array}{l}5.94 \\
6.25 \\
6.55 \\
6.83 \\
7.23 \\
7.73 \\
8.25 \\
8.77 \\
9.24 \\
9.72 \\
10.23 \\
10.75 \\
11.26 \\
11.76 \\
12.40 \\
13.20 \\
14.03 \\
14.86 \\
15.62 \\
16.45 \\
17.44 \\
18.51 \\
19.58 \\
20.53 \\
21.43 \\
22.40 \\
23.43\end{array}$ & $\begin{array}{r}3.84 \mathrm{E}-09 \\
3.40 \mathrm{E}-09 \\
3.19 \mathrm{E}-09 \\
3.09 \mathrm{E}-09 \\
2.88 \mathrm{E}-09 \\
2.41 \mathrm{E}-09 \\
1.90 \mathrm{E}-09 \\
1.70 \mathrm{E}-09 \\
1.61 \mathrm{E}-09 \\
1.47 \mathrm{E}-09 \\
1.38 \mathrm{E}-09 \\
1.47 \mathrm{E}-09 \\
1.59 \mathrm{E}-09 \\
1.72 \mathrm{E}-09 \\
2.33 \mathrm{E}-09 \\
4.15 \mathrm{E}-09 \\
5.98 \mathrm{E}-09 \\
5.77 \mathrm{E}-09 \\
4.01 \mathrm{E}-09 \\
1.98 \mathrm{E}-09 \\
6.30 \mathrm{E}-10 \\
1.30 \mathrm{E}-10 \\
1.31 \mathrm{E}-11 \\
-9.67 \mathrm{E}-12 \\
-1.54 \mathrm{E}-11 \\
-1.74 \mathrm{E}-11 \\
-1.54 \mathrm{E}-11\end{array}$ & $\begin{array}{r}4.02 \mathrm{E}-09 \\
3.60 \mathrm{E}-09 \\
3.41 \mathrm{E}-09 \\
3.27 \mathrm{E}-09 \\
3.04 \mathrm{E}-09 \\
2.60 \mathrm{E}-09 \\
2.06 \mathrm{E}-09 \\
1.83 \mathrm{E}-09 \\
1.75 \mathrm{E}-09 \\
1.60 \mathrm{E}-09 \\
1.51 \mathrm{E}-09 \\
1.60 \mathrm{E}-09 \\
1.72 \mathrm{E}-09 \\
1.85 \mathrm{E}-09 \\
2.45 \mathrm{E}-09 \\
4.25 \mathrm{E}-09 \\
6.07 \mathrm{E}-09 \\
5.85 \mathrm{E}-09 \\
4.07 \mathrm{E}-09 \\
2.02 \mathrm{E}-09 \\
6.47 \mathrm{E}-10 \\
1.39 \mathrm{E}-10 \\
2.01 \mathrm{E}-11 \\
-3.53 \mathrm{E}-12 \\
-1.04 \mathrm{E}-11 \\
-1.20 \mathrm{E}-11 \\
-9.44 \mathrm{E}-12\end{array}$ \\
\hline
\end{tabular}




\section{Table A.10 (Cont'd) \\ Gamma-Ray Data \\ Detector 10 in Fig. 4}

Detector Coordinates $(\mathrm{cm}): x=79.0, y=0.0, z^{\prime}=151.0$

\begin{tabular}{|c|c|c|c|c|c|}
\hline \multirow{2}{*}{$\begin{array}{l}\text { Energy } \\
\text { (MeV) }\end{array}$} & \multicolumn{2}{|c|}{$\underline{F} \underline{1} \underline{u} \in \underline{n} \underline{c} \underline{e}$ ( $(\underline{M} \underline{\mathrm{e}}-1$} & \multirow{2}{*}{$\begin{array}{l}\text { Energy } \\
(\mathrm{MeV})\end{array}$} & \multicolumn{2}{|c|}{ FI } \\
\hline & Confidence & Interval & & Confidence & Interval \\
\hline $\begin{array}{l}0.72 \\
0.76 \\
0.80 \\
0.84\end{array}$ & $\begin{array}{l}1.24 \mathrm{E}-07 \\
2.07 \mathrm{E}-07 \\
2.48 \mathrm{E}-07 \\
2.49 \mathrm{E}-07\end{array}$ & $\begin{array}{l}2.14 \mathrm{E}-07 \\
2.44 \mathrm{E}-07 \\
2.58 \mathrm{E}-07 \\
2.54 \mathrm{E}-07\end{array}$ & $\begin{array}{l}3.93 \\
4.06 \\
4.19 \\
4.31\end{array}$ & $\begin{array}{l}2.67 \mathrm{E}-08 \\
2.47 \mathrm{E}-08 \\
2.35 \mathrm{E}-08 \\
2.26 \mathrm{E}-08\end{array}$ & $\begin{array}{l}2.85 \mathrm{E}-08 \\
2.66 \mathrm{E}-08 \\
2.55 \mathrm{E}-08 \\
2.50 \mathrm{E}-08\end{array}$ \\
\hline 0.88 & $2.28 \mathrm{E}-07$ & $2.32 \mathrm{E}-07$ & 4.44 & $2.21 \mathrm{E}-08$ & $2.49 \mathrm{E}-08$ \\
\hline 0.92 & $2.00 \mathrm{E}-07$ & $2.03 \mathrm{E}-07$ & 4.57 & $2.24 \mathrm{E}-08$ & $2.54 \mathrm{E}-0.8$ \\
\hline 0.96 & $1.73 \mathrm{E}-07$ & $1.76 \mathrm{E}-07$ & 4.70 & $2.35 \mathrm{E}-08$ & $2.66 \mathrm{E}-08$ \\
\hline 1.00 & $1.52 \mathrm{E}-07$ & $1.55 \mathrm{E}-07$ & 4.83 & $2.49 \mathrm{E}-08$ & $2.81 E-08$ \\
\hline 1.04 & $1.39 \mathrm{E}-07$ & $1.41 \mathrm{E}-07$ & 4.99 & $2.49 \mathrm{E}-08$ & $2.87 \mathrm{E}-08$ \\
\hline 1.08 & $1.33 \mathrm{E}-07$ & $1.35 \mathrm{E}-07$ & 5.15 & $2.11 \mathrm{E}-08$ & $2.52 \mathrm{E}-08$ \\
\hline 1.13 & $1.33 \mathrm{E}-07$ & $1.35 \mathrm{E}-07$ & 5.32 & $1.44 \mathrm{E}-08$ & $1.85 \mathrm{E}-08$ \\
\hline 1.17 & $1.36 \mathrm{E}-07$ & $1.38 \mathrm{E}-07$ & 5.50 & $9.98 \mathrm{E}-09$ & $1.45 \mathrm{E}-08$ \\
\hline 1.22 & $1.36 \mathrm{E}-07$ & $1.39 \mathrm{E}-07$ & 5.67 & $1.33 \mathrm{E}-08$ & $1.82 E-08$ \\
\hline 1.28 & 1. $32 \mathrm{E}-07$ & $1.35 \mathrm{E}-07$ & 5.83 & $2.36 \mathrm{E}-08$ & $2.81 \mathrm{E}-08$ \\
\hline 1.33 & $1.25 \mathrm{E}-07$ & $1.28 \mathrm{E}-07$ & 6.00 & $3.44 \mathrm{E}-08$ & $3.85 E-08$ \\
\hline 1.38 & $1.18 \mathrm{E}-07$ & $1.19 \mathrm{E}-07$ & 6.18 & $3.96 \mathrm{E}-08$ & $4.37 E-08$ \\
\hline 1.42 & $1.1 .0 \mathrm{E}-07$ & $1.12 \mathrm{E}-07$ & 6.35 & $3.73 \mathrm{E}-08$ & $4.12 \mathrm{E}-08$ \\
\hline 1.47 & $1.03 \mathrm{E}-07$ & $1.05 \mathrm{E}-07$ & 6.51 & $3.04 \mathrm{E}-08$ & $3.38 \mathrm{E}-08$ \\
\hline 1.53 & $9.64 E-08$ & $9.85 \mathrm{E}-08$ & 6.70 & $2.29 \mathrm{E}-08$ & $2.57 \mathrm{E}-08$ \\
\hline 1.58 & $9.24 E-08$ & $9.45 \mathrm{E}-08$ & 6.90 & $1.92 \mathrm{E}-08$ & $2.16 \mathrm{E}-08$ \\
\hline $\begin{array}{l}1.64 \\
1.72 \\
1.80\end{array}$ & $\begin{array}{l}9.09 \mathrm{E}-08 \\
9.39 \mathrm{E}-08 \\
1.01 \mathrm{E}-07\end{array}$ & $\begin{array}{l}9.29 \mathrm{E}-08 \\
9.57 \mathrm{E}-08 \\
1.02 \mathrm{E}-07\end{array}$ & $\begin{array}{l}7.10 \\
7.30 \\
7.50\end{array}$ & $\begin{array}{l}2.02 \mathrm{E}-08 \\
2.27 \mathrm{E}-08 \\
2.32 \mathrm{E}-08\end{array}$ & $\begin{array}{l}2.26 \mathrm{E}-08 \\
2.48 \mathrm{E}-08 \\
2.52 \mathrm{E}-08\end{array}$ \\
\hline 1.88 & $1.08 \mathrm{E}-07$ & $1.10 \mathrm{E}-07$ & 7.70 & $2.04 E-08$ & $2.23 \mathrm{E}-08$ \\
\hline 1.96 & $1.12 \mathrm{E}-07$ & $1.14 \mathrm{E}-07$ & 7.90 & $1.53 \mathrm{E}-08$ & $1.70 \mathrm{E}-08$ \\
\hline 2.04 & $1.12 \mathrm{E}-07$ & $1.14 \mathrm{E}-07$ & 8.10 & $9.78 \mathrm{E}-09$ & $1.13 E-08$ \\
\hline 2.12 & $1.10 \mathrm{E}-07$ & $1.11 \mathrm{E}-07$ & 8.30 & $5.46 \mathrm{E}-09$ & $6.78 \mathrm{E}-09$ \\
\hline 2.20 & $1.05 \mathrm{E}-07$ & $1.06 \mathrm{E}-07$ & 8.50 & $3.06 \mathrm{E}-09$ & $4.19 E-09$ \\
\hline 2.28 & $9.45 \mathrm{E}-08$ & $9.62 \mathrm{E}-08$ & 8.73 & $2.13 E-09$ & $3.07 E-09$ \\
\hline 2.36 & $8.00 \mathrm{E}-08$ & $8.15 \mathrm{E}-08$ & 8.98 & $2.12 \mathrm{E}-09$ & $2.94 E-09$ \\
\hline 2.45 & $6.30 \mathrm{E}-08$ & $6.45 \mathrm{E}-08$ & 9.23 & $2.02 \mathrm{E}-09$ & $2.71 \mathrm{E}-09$ \\
\hline 2.55 & $4.95 \mathrm{E}-08$ & $5.08 \mathrm{E}-08$ & 9.48 & $1.46 \mathrm{E}-09$ & $2.08 \mathrm{E}-09$ \\
\hline 2.65 & $4.28 \mathrm{E}-08$ & $4.42 \mathrm{E}-08$ & 9.73 & $6.60 \mathrm{E}-10$ & $1.17 \mathrm{E}-09$ \\
\hline 2.75 & $3.99 \mathrm{E}-08$ & $4.14 \mathrm{E}-08$ & 9.98 & $-1.49 \mathrm{E}-11$ & $4.46 \mathrm{E}-10$ \\
\hline 2.85 & $3.74 \mathrm{E}-08$ & $3.88 \mathrm{E}-08$ & 10.30 & $-3.92 \mathrm{E}-10$ & $5.68 \mathrm{E}-11$ \\
\hline 2.95 & $3.45 \mathrm{E}-08$ & $3.59 \mathrm{E}-08$ & 10.70 & $-2.96 \mathrm{E}-10$ & $1.88 \mathrm{E}-10$ \\
\hline 3.05 & $3.24 \mathrm{E}-08$ & $3.38 \mathrm{E}-08$ & 11.10 & $-1.08 \mathrm{E}-10$ & $4.24 E-10$ \\
\hline 3.15 & $3.14 E-08$ & $3.29 \mathrm{E}-08$ & 11.50 & $-8.64 E-11$ & $4.38 \mathrm{E}-10$ \\
\hline 3.25 & $3.09 \mathrm{E}-08$ & $3.25 \mathrm{E}-08$ & 11.90 & $-8.29 \mathrm{E}-11$ & $3.67 \mathrm{E}-10$ \\
\hline 3.35 & $3.08 \mathrm{E}-08$ & $3.23 \mathrm{E}-08$ & 12.30 & $1.76 \mathrm{E}-11$ & $3.46 \mathrm{E}-10$ \\
\hline 3.45 & $3.09 E-08$ & $3.24 \mathrm{E}-08$ & 12.70 & $1.49 \mathrm{E}-10$ & $3.72 \mathrm{E}-10$ \\
\hline 3.55 & $3.12 E-08$ & $3.28 \mathrm{E}-08$ & 13.10 & $1.88 \mathrm{E}-10$ & $4.07 \mathrm{E}-10$ \\
\hline 3.66 & $3.08 \mathrm{E}-08$ & $3.26 \mathrm{E}-08$ & 13.50 & $9.49 \mathrm{E}-11$ & $3.77 \mathrm{E}-10$ \\
\hline 3.79 & $2.92 \mathrm{E}-08$ & $3.09 \mathrm{E}-08$ & 13.90 & $6.34 \mathrm{E}-12$ & $2.99 \mathrm{E}-10$ \\
\hline
\end{tabular}


Table A.11

Neutron Data

Detector 11 in Fig. 4

Detector Coordinates $(\mathrm{cm}): x=98.0, y=0.0, z^{\prime}=151.0$

\begin{tabular}{|c|c|c|c|c|c|}
\hline $\begin{array}{l}\text { Energy } \\
(\mathrm{MeV})\end{array}$ & 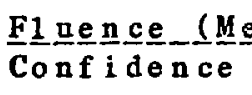 & 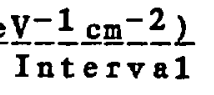 & $\begin{array}{l}\text { Energy } \\
\text { ( } \mathrm{MeV})\end{array}$ & 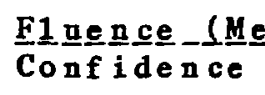 & $=\frac{v^{-1}}{I n t \in}-\frac{c}{-2}-\frac{2}{a 1}$ \\
\hline $\begin{array}{l}0.91 \\
1.01 \\
1.11 \\
1.21 \\
1.31 \\
1.41 \\
1.51 \\
1.61 \\
1.71 \\
1.81 \\
1.94 \\
2.11 \\
2.31 \\
2.50 \\
2.69 \\
2.90 \\
3.10 \\
3.30 \\
3.51 \\
3.71 \\
3.90 \\
4.15 \\
4.46 \\
4.75 \\
5.06 \\
5.36 \\
5.64\end{array}$ & $\begin{array}{l}9.19 \mathrm{E}-08 \\
8.46 \mathrm{E}-08 \\
7.54 \mathrm{E}-08 \\
6.72 \mathrm{E}-08 \\
6.01 \mathrm{E}-08 \\
5.42 \mathrm{E}-08 \\
4.92 \mathrm{E}-08 \\
4.42 \mathrm{E}-08 \\
3.94 \mathrm{E}-08 \\
3.51 \mathrm{E}-08 \\
3.12 \mathrm{E}-08 \\
2.83 \mathrm{E}-08 \\
2.50 \mathrm{E}-08 \\
2.09 \mathrm{E}-08 \\
1.73 \mathrm{E}-08 \\
1.42 \mathrm{E}-08 \\
1.15 \mathrm{E}-08 \\
9.10 \mathrm{E}-09 \\
7.15 \mathrm{E}-09 \\
6.12 \mathrm{E}-09 \\
5.78 \mathrm{E}-09 \\
5.36 \mathrm{E}-09 \\
4.57 \mathrm{E}-09 \\
3.95 \mathrm{E}-09 \\
3.59 \mathrm{E}-09 \\
3.19 \mathrm{E}-09 \\
2.83 \mathrm{E}-09\end{array}$ & $\begin{array}{l}9.27 \mathrm{E}-08 \\
8.51 \mathrm{E}-08 \\
7.60 \mathrm{E}-08 \\
6.78 \mathrm{E}-08 \\
6.06 \mathrm{E}-08 \\
5.46 \mathrm{E}-08 \\
4.97 \mathrm{E}-08 \\
4.46 \mathrm{E}-08 \\
3.97 \mathrm{E}-08 \\
3.55 \mathrm{E}-08 \\
3.16 \mathrm{E}-08 \\
2.86 \mathrm{E}-08 \\
2.53 \mathrm{E}-08 \\
2.12 \mathrm{E}-08 \\
1.75 \mathrm{E}-08 \\
1.45 \mathrm{E}-08 \\
1.18 \mathrm{E}-08 \\
9.32 \mathrm{E}-09 \\
7.40 \mathrm{E}-09 \\
6.39 \mathrm{E}-09 \\
5.97 \mathrm{E}-09 \\
5.56 \mathrm{E}-09 \\
4.69 \mathrm{E}-09 \\
4.08 \mathrm{E}-09 \\
3.73 \mathrm{E}-09 \\
3.34 \mathrm{E}-09 \\
2.97 \mathrm{E}-09\end{array}$ & $\begin{array}{l}5.94 \\
6.25 \\
6.55 \\
6.83 \\
7.23 \\
7.73 \\
8.25 \\
8.77 \\
9.24 \\
9.72 \\
10.23 \\
10.75 \\
11.26 \\
11.76 \\
12.40 \\
13.20 \\
14.03 \\
14.86 \\
15.62 \\
16.45 \\
17.44 \\
18.51 \\
19.58 \\
20.53 \\
21.43 \\
22.40 \\
23.43\end{array}$ & $\begin{array}{r}2.54 \mathrm{E}-09 \\
2.28 \mathrm{E}-09 \\
2.10 \mathrm{E}-09 \\
1.97 \mathrm{E}-09 \\
1.79 \mathrm{E}-09 \\
1.55 \mathrm{E}-09 \\
1.26 \mathrm{E}-09 \\
1.06 \mathrm{E}-09 \\
9.74 \mathrm{E}-10 \\
9.31 \mathrm{E}-10 \\
9.34 \mathrm{E}-10 \\
9.69 \mathrm{E}-10 \\
9.75 \mathrm{E}-10 \\
1.03 \mathrm{E}-09 \\
1.36 \mathrm{E}-09 \\
2.21 \mathrm{E}-09 \\
3.00 \mathrm{E}-09 \\
2.87 \mathrm{E}-09 \\
2.00 \mathrm{E}-09 \\
9.89 \mathrm{E}-10 \\
3.12 \mathrm{E}-10 \\
6.31 \mathrm{E}-11 \\
5.79 \mathrm{E}-12 \\
-4.97 \mathrm{E}-12 \\
-7.61 \mathrm{E}-12 \\
-8.62 \mathrm{E}-12 \\
-7.71 \mathrm{E}-12\end{array}$ & $\begin{array}{r}2.66 \mathrm{E}-09 \\
2.41 \mathrm{E}-09 \\
2.23 \mathrm{E}-09 \\
2.08 \mathrm{E}-09 \\
1.89 \mathrm{E}-09 \\
1.67 \mathrm{E}-09 \\
1.37 \mathrm{E}-09 \\
1.15 \mathrm{E}-09 \\
1.06 \mathrm{E}-09 \\
1.01 \mathrm{E}-09 \\
1.01 \mathrm{E}-09 \\
1.05 \mathrm{E}-09 \\
1.06 \mathrm{E}-09 \\
1.10 \mathrm{E}-09 \\
1.43 \mathrm{E}-09 \\
2.27 \mathrm{E}-09 \\
3.06 \mathrm{E}-09 \\
2.92 \mathrm{E}-09 \\
2.04 \mathrm{E}-09 \\
1.01 \mathrm{E}-09 \\
3.22 \mathrm{E}-10 \\
6.75 \mathrm{E}-11 \\
9.54 \mathrm{E}-12 \\
-1.63 \mathrm{E}-12 \\
-4.89 \mathrm{E}-12 \\
-5.69 \mathrm{E}-12 \\
-4.48 \mathrm{E}-12\end{array}$ \\
\hline
\end{tabular}


Table A.11 (Cont'd)

Gamma-Ray Data

Detector 11 in Fig. 4

Detector Coordinates $(\mathrm{cm}): x=98.0, y=0.0, z^{\prime}=151.0$

\begin{tabular}{|c|c|c|c|c|c|}
\hline $\begin{array}{l}\text { Energy } \\
(\mathrm{MeV})\end{array}$ & 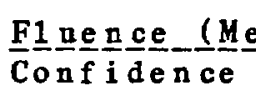 & $\frac{V^{-1}}{\text { Int }}$ & $\begin{array}{l}\text { Energy } \\
(\mathrm{MeV})\end{array}$ & 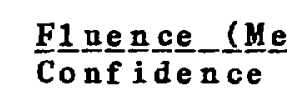 & 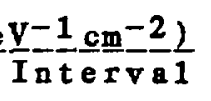 \\
\hline 0.72 & $7.76 \mathrm{E}-08$ & $1.39 \mathrm{E}-07$ & 3.93 & $1.91 \mathrm{E}-08$ & $2.03 \mathrm{E}-08$ \\
\hline 0.76 & $1.36 \mathrm{E}-07$ & $1.61 \mathrm{E}-07$ & 4.06 & $1.65 \mathrm{E}-08$ & $1.79 \mathrm{E}-08$ \\
\hline 0.80 & $1.66 \mathrm{E}-07$ & $1.74 \mathrm{E}-07$ & 4.19 & $1.46 \mathrm{E}-08$ & $1.60 \mathrm{E}-08$ \\
\hline 0.84 & $1.70 \mathrm{E}-07$ & $1.73 \mathrm{E}-07$ & 4.31 & $1.37 \mathrm{E}-08$ & $1.53 \mathrm{E}-08$ \\
\hline 0.88 & $1.57 \mathrm{E}-07$ & 1.59E-07 & 4.44 & $1.38 \mathrm{E}-08$ & $1.57 \mathrm{E}-08$ \\
\hline 0.92 & $1.37 \mathrm{E}-07$ & $1.39 \mathrm{E}-07$ & 4.57 & $1.51 \mathrm{E}-08$ & $1.72 \mathrm{E}-08$ \\
\hline 0.96 & $1.17 \mathrm{E}-07$ & $1.20 \mathrm{E}-07$ & 4.70 & $1.70 \mathrm{E}-08$ & $1.92 \mathrm{E}-08$ \\
\hline 1.00 & $1.03 \mathrm{E}-07$ & $1.05 \mathrm{E}-07$ & 4.83 & $1.84 \mathrm{E}-08$ & $2.07 \mathrm{E}-08$ \\
\hline 1.04 & $9.42 \mathrm{E}-08$ & $9.59 \mathrm{E}-08$ & 4.99 & $1.74 \mathrm{E}-08$ & $2.02 \mathrm{E}-08$ \\
\hline 1.08 & $8.96 \mathrm{E}-08$ & $9.12 \mathrm{E}-08$ & 5.15 & $1.28 \mathrm{E}-08$ & $1.57 \mathrm{E}-08$ \\
\hline 1.13 & $8.88 \mathrm{E}-08$ & $9.05 \mathrm{E}-08$ & 5.32 & $7.39 E-09$ & $1.03 \mathrm{E}-08$ \\
\hline 1.17 & $9.08 \mathrm{E}-08$ & $9.25 \mathrm{E}-08$ & 5.50 & $5.55 \mathrm{E}-09$ & $8.75 \mathrm{E}-09$ \\
\hline 1.22 & $9.17 \mathrm{E}-08$ & $9.36 \mathrm{E}-08$ & 5.67 & $9.51 \mathrm{E}-09$ & $1.29 \mathrm{E}-08$ \\
\hline 1.28 & $8.99 \mathrm{E}-08$ & $9.19 \mathrm{E}-08$ & 5.83 & $1.72 \mathrm{E}-08$ & $2.04 \mathrm{E}-08$ \\
\hline 1.33 & $8.65 \mathrm{E}-08$ & $8.81 \mathrm{E}-08$ & 6.00 & $2.40 \mathrm{E}-08$ & $2.69 \mathrm{E}-08$ \\
\hline 1.38 & $8.21 \mathrm{E}-08$ & $8.35 \mathrm{E}-08$ & 6.18 & $2.67 \mathrm{E}-08$ & $2.97 \mathrm{E}-08$ \\
\hline 1.42 & $7.69 \mathrm{E}-08$ & $7.85 \mathrm{E}-08$ & 6.35 & $2.49 \mathrm{E}-08$ & $2.77 \mathrm{E}-08$ \\
\hline 1.47 & $7.12 \mathrm{E}-08$ & $7.28 \mathrm{E}-08$ & -6.51 & $2.06 \mathrm{E}-08$ & $2.31 \mathrm{E}-08$ \\
\hline 1.53 & $6.59 \mathrm{E}-08$ & $6.74 \mathrm{E}-08$ & 6.70 & $1.62 \mathrm{E}-08$ & $1.82 E-08$ \\
\hline 1.58 & $6.21 \mathrm{E}-08$ & $6.36 \mathrm{E}-08$ & 6.90 & $1.41 \mathrm{E}-08$ & $1.60 \mathrm{E}-08$ \\
\hline 1.64 & $6.04 \mathrm{E}-08$ & $6.18 \mathrm{E}-08$ & 7.10 & $1.49 \mathrm{E}-08$ & $1.66 \mathrm{E}-08$ \\
\hline $\begin{array}{l}1.72 \\
1.80\end{array}$ & $\begin{array}{l}6.25 \mathrm{E}-08 \\
6.75 \mathrm{E}-08\end{array}$ & $\begin{array}{l}6.38 \mathrm{E}-08 \\
6.88 \mathrm{E}-08\end{array}$ & & $\begin{array}{l}1.65 \mathrm{E}-08 \\
1.69 \mathrm{E}-08\end{array}$ & $\begin{array}{l}1.80 \mathrm{E}-08 \\
1.83 \mathrm{E}-08\end{array}$ \\
\hline 1.88 & $7.31 \mathrm{E}-08$ & $7.44 \mathrm{E}-08$ & 7.70 & $1.52 \mathrm{E}-08$ & $1.65 \mathrm{E}-08$ \\
\hline 1.96 & $7.64 \mathrm{E}-08$ & $7.78 \mathrm{E}-08$ & 7.90 & $1.19 \mathrm{E}-08$ & $1.30 E-08$ \\
\hline 2.04 & $7.68 \mathrm{E}-08$ & $7.82 \mathrm{E}-08$ & 8.10 & $8.03 E-09$ & $9.06 \mathrm{E}-09$ \\
\hline 2.12 & $7.62 \mathrm{E}-08$ & $7.76 \mathrm{E}-08$ & 8.30 & $4.73 \mathrm{E}-09$ & $5.64 \mathrm{E}-09$ \\
\hline 2.20 & $7.45 \mathrm{E}-08$ & $7.58 \mathrm{E}-08$ & 8.50 & $2.61 \mathrm{E}-09$ & $3.39 \mathrm{E}-09$ \\
\hline 2.28 & $6.89 \mathrm{E}-08$ & $7.01 \mathrm{E}-08$ & 8.73 & $1.57 \mathrm{E}-09$ & $2.25 \mathrm{E}-09$ \\
\hline 2.36 & $5.89 \mathrm{E}-08$ & $6.00 \mathrm{E}-08$ & 8.98 & $1.43 \mathrm{E}-09$ & $2.02 \mathrm{E}-09$ \\
\hline 2.45 & $4.62 \mathrm{E}-08$ & $4.73 E-08$ & 9.23 & $1.47 \mathrm{E}-09$ & $1.97 \mathrm{E}-09$ \\
\hline 2.55 & $3.56 \mathrm{E}-08$ & $3.66 \mathrm{E}-08$ & 9.48 & $1.24 \mathrm{E}-09$ & $1.67 \mathrm{E}-09$ \\
\hline 2.65 & $3.00 \mathrm{E}-08$ & $3.10 \mathrm{E}-08$ & 9.73 & $7.74 \mathrm{E}-10$ & $1.16 \mathrm{E}-09$ \\
\hline 2.75 & $2.77 \mathrm{E}-08$ & $2.88 \mathrm{E}-08$ & 9.98 & $2.58 \mathrm{E}-10$ & $6.12 \mathrm{E}-10$ \\
\hline 2.85 & $2.64 \mathrm{E}-08$ & $2.74 \mathrm{E}-08$ & 10.30 & $-1.68 E-10$ & $1.75 \mathrm{E}-10$ \\
\hline 2.95 & $2.46 \mathrm{E}-08$ & $2.56 \mathrm{E}-08$ & 10.70 & $-2.78 \mathrm{E}$ & $7.30 \mathrm{E}-11$ \\
\hline 3.05 & $2.25 \mathrm{E}-08$ & $2.35 \mathrm{E}-08$ & 11.10 & $-1.35 \mathrm{E}-10$ & $2.29 \mathrm{E}-10$ \\
\hline 3.15 & $2.07 \mathrm{E}-08$ & $2.17 \mathrm{E}-08$ & 11.50 & $1.43 \mathrm{E}-11$ & $3.71 \mathrm{E}-10$ \\
\hline 3.25 & $1.97 \mathrm{E}-08$ & $2.07 \mathrm{E}-08$ & 11.90 & $6.28 \mathrm{E}-11$ & $3.72 \mathrm{E}-10$ \\
\hline 3.35 & $1.96 \mathrm{E}-08$ & $2.06 \mathrm{E}-08$ & 12.30 & $3.42 \mathrm{E}-11$ & $2.70 \mathrm{E}-10$ \\
\hline 3.45 & $2.02 \mathrm{E}-08$ & $2.12 \mathrm{E}-08$ & 12.70 & $6.38 \mathrm{E}-12$ & $1.96 \mathrm{E}-10$ \\
\hline 3.55 & $2.10 \mathrm{E}-08$ & $2.21 \mathrm{E}-0 \mathrm{~B}$ & 13.10 & $-2.76 \mathrm{E}-12$ & $2.11 \mathrm{E}-10$ \\
\hline 3.66 & $\begin{array}{l}2.16 \mathrm{E}-08 \\
2.11 \mathrm{E}-08\end{array}$ & $\begin{array}{l}2.28 \mathrm{E}-0 \mathrm{~B} \\
2.23 \mathrm{~B}-\mathrm{OB}\end{array}$ & $\begin{array}{l}13.50 \\
13.90\end{array}$ & $-2.17 \mathrm{E}-11$ & $2.13 \mathrm{E}-10$ \\
\hline & & & & $-1.47 \mathrm{E}-11$ & $1.72 \mathrm{E}-10$ \\
\hline
\end{tabular}


Table A. 12

Neutron Data

Detector 12 in Fig. 4

Detector Coodinates $(\mathrm{cm}): \quad x=119.0, y=0.0, z^{\prime}=151.0$

\begin{tabular}{|c|c|c|c|c|c|}
\hline $\begin{array}{l}\text { Energy } \\
(\mathrm{MeV})\end{array}$ & 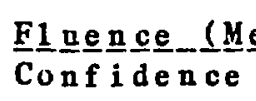 & 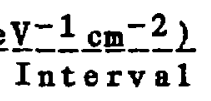 & $\begin{array}{l}\text { Energy } \\
(\mathrm{MeV})\end{array}$ & 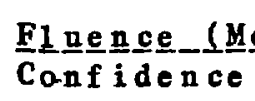 & $=\frac{\left.v^{-1} \operatorname{cm}^{-2}\right)}{\operatorname{Int} \operatorname{cr} \text { a } 1}$ \\
\hline $\begin{array}{l}0.91 \\
1.01 \\
1.11 \\
1.21 \\
1.31 \\
1.41 \\
1.51 \\
1.61 \\
1.71 \\
1.81 \\
1.94 \\
2.11 \\
2.31 \\
2.50 \\
2.69 \\
2.90 \\
3.10 \\
3.30 \\
3.51 \\
3.71 \\
3.90 \\
4.15 \\
4.46 \\
4.75 \\
5.06 \\
5.36 \\
5.64\end{array}$ & $\begin{array}{l}5.36 \mathrm{E}-08 \\
4.97 \mathrm{E}-08 \\
4.45 \mathrm{E}-08 \\
3.95 \mathrm{E}-08 \\
3.53 \mathrm{E}-08 \\
3.20 \mathrm{E}-08 \\
2.92 \mathrm{E}-08 \\
2.62 \mathrm{E}-08 \\
2.32 \mathrm{E}-08 \\
2.04 \mathrm{E}-08 \\
1.80 \mathrm{E}-08 \\
1.63 \mathrm{E}-08 \\
1.45 \mathrm{E}-08 \\
1.23 \mathrm{E}-08 \\
1.00 \mathrm{E}-08 \\
7.93 \mathrm{E}-09 \\
6.26 \mathrm{E}-09 \\
5.03 \mathrm{E}-09 \\
4.11 \mathrm{E}-09 \\
3.54 \mathrm{E}-09 \\
3.20 \mathrm{E}-09 \\
2.82 \mathrm{E}-09 \\
2.45 \mathrm{E}-09 \\
2.17 \mathrm{E}-09 \\
1.94 \mathrm{E}-09 \\
1.69 \mathrm{E}-09 \\
1.45 \mathrm{E}-09\end{array}$ & $\begin{array}{l}5.41 \mathrm{E}-08 \\
5.00 \mathrm{E}-08 \\
4.48 \mathrm{E}-08 \\
3.98 \mathrm{E}-08 \\
3.56 \mathrm{E}-08 \\
3.23 \mathrm{E}-08 \\
2.95 \mathrm{E}-08 \\
2.64 \mathrm{E}-08 \\
2.34 \mathrm{E}-08 \\
2.07 \mathrm{E}-08 \\
1.83 \mathrm{E}-08 \\
1.65 \mathrm{E}-08 \\
1.47 \mathrm{E}-08 \\
1.25 \mathrm{E}-08 \\
1.02 \mathrm{E}-0.8 \\
8.07 \mathrm{E}-09 \\
6.44 \mathrm{E}-09 \\
5.16 \mathrm{E}-09 \\
4.27 \mathrm{E}-09 \\
3.70 \mathrm{E}-09 \\
3.32 \mathrm{E}-09 \\
2.94 \mathrm{E}-09 \\
2.52 \mathrm{E}-09 \\
2.25 \mathrm{E}-09 \\
2.02 \mathrm{E}-09 \\
1.78 \mathrm{E}-09 \\
1.53 \mathrm{E}-09\end{array}$ & $\begin{array}{l}5.94 \\
6.25 \\
6.55 \\
6.83 \\
7.23 \\
7.73 \\
8.25 \\
8.77 \\
9.24 \\
9.72 \\
10.23 \\
1.0 .75 \\
11.26 \\
11.76 \\
12.40 \\
13.20 \\
14.03 \\
14.86 \\
15.62 \\
16.45 \\
17.44 \\
18.51 \\
19.58 \\
20.53 \\
21.43 \\
22.40 \\
23.43\end{array}$ & $\begin{array}{r}1.24 \mathrm{E}-09 \\
1.10 \mathrm{E}-09 \\
1.01 \mathrm{E}-09 \\
9.45 \mathrm{E}-10 \\
8.30 \mathrm{E}-10 \\
7.00 \mathrm{E}-10 \\
5.98 \mathrm{E}-10 \\
5.30 \mathrm{E}-10 \\
4.97 \mathrm{E}-10 \\
4.74 \mathrm{E}-10 \\
4.59 \mathrm{E}-10 \\
4.64 \mathrm{E}-10 \\
4.71 \mathrm{E}-10 \\
4.98 \mathrm{E}-10 \\
6.53 \mathrm{E}-10 \\
9.99 \mathrm{E}-10 \\
1.14 \mathrm{E}-09 \\
8.96 \mathrm{E}-10 \\
5.45 \mathrm{E}-10 \\
2.44 \mathrm{E}-10 \\
7.09 \mathrm{E}-11 \\
1.11 \mathrm{E}-11 \\
-2.82 \mathrm{E}-12 \\
-4.83 \mathrm{E}-12 \\
-4.63 \mathrm{E}-12 \\
-4.71 \mathrm{E}-12 \\
-4.14 \mathrm{E}-12\end{array}$ & $\begin{array}{r}1.31 \mathrm{E}-09 \\
1.17 \mathrm{E}-09 \\
1.09 \mathrm{E}-09 \\
1.01 \mathrm{E}-09 \\
8.8 \mathrm{~B}-10 \\
7.67 \mathrm{E}-10 \\
6.59 \mathrm{E}-10 \\
5.79 \mathrm{E}-10 \\
5.47 \mathrm{E}-10 \\
5.17 \mathrm{E}-10 \\
5.02 \mathrm{E}-10 \\
5.08 \mathrm{E}-10 \\
5.15 \mathrm{E}-10 \\
5.39 \mathrm{E}-10 \\
6.95 \mathrm{E}-10 \\
1.03 \mathrm{E}-09 \\
1.16 \mathrm{E}-09 \\
9.16 \mathrm{E}-10 \\
5.60 \mathrm{E}-10 \\
2.54 \mathrm{E}-10 \\
7.67 \mathrm{E}-11 \\
1.56 \mathrm{E}-11 \\
1.90 \mathrm{E}-12 \\
-4.23 \mathrm{E}-13 \\
-9.70 \mathrm{E}-13 \\
-7.28 \mathrm{E}-13 \\
2.64 \mathrm{E}-13\end{array}$ \\
\hline
\end{tabular}


Table. A.12 (Cont'd)

Gamma-Ray Data

Detector 12 in Fig. 4

Detector Coordinates $(\mathrm{cm}): x=119.0, y=0.0, z^{\prime}=151.0$

\begin{tabular}{|c|c|c|c|c|c|}
\hline \multirow{2}{*}{$\begin{array}{l}\text { Energy } \\
(\mathrm{MeV})\end{array}$} & \multicolumn{2}{|c|}{$\underline{F} \underline{1} \underline{\mathrm{n}} \underline{\mathrm{n}} \mathrm{ce}$ ( $(\underline{\mathrm{M}} \mathrm{eV}-1 \mathrm{~cm}-2)$} & \multirow{2}{*}{$\begin{array}{l}\text { Energy } \\
(\mathrm{MeV})\end{array}$} & \multicolumn{2}{|c|}{ 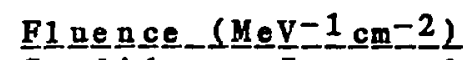 } \\
\hline & Confidence & Interva 1 & & Confidence & Interval \\
\hline 0.72 & $5.18 \mathrm{E}-08$ & $8.79 E-08$ & 3.93 & $1.05 \mathrm{E}-08$ & $1.13 \mathrm{E}-08$ \\
\hline 0.76 & $8.08 \mathrm{E}-08$ & $9.55 \mathrm{E}-08$ & 4.06 & $9.09 \mathrm{E}-09$ & $9.95 \mathrm{E}-09$ \\
\hline 0.80 & $9.61 \mathrm{E}-08$ & $1.00 \mathrm{E}-07$ & 4.19 & $8.13 \mathrm{E}-09$ & $9.04 \mathrm{E}-09$ \\
\hline 0.84 & $9.70 \mathrm{E}-08$ & $9.87 \mathrm{E}-08$ & 4.31 & $7.75 \mathrm{E}-09$ & $8.80 \mathrm{E}-09$ \\
\hline 0.88 & $8.77 E-08$ & $8.91 \mathrm{E}-08$ & 4.44 & $7.91 \mathrm{E}-09$ & $9.16 \mathrm{E}-09$ \\
\hline 0.92 & $7.47 \mathrm{E}-08$ & $7.60 \mathrm{E}-08$ & 4.57 & $8.65 \mathrm{E}-09$ & $1.00 \mathrm{E}-08$ \\
\hline 0.96 & $6.3 \cdot 1 \mathrm{E}-08$ & $6.43 \mathrm{E}-08$ & 4.70 & $9.83 \mathrm{E}-09$ & $1.12 \mathrm{E}-08$ \\
\hline 1.00 & $5.52 E-08$ & $5.63 \mathrm{E}-08$ & 4.83 & $1.07 \mathrm{E}-08$ & $1.22 \mathrm{E}-08$ \\
\hline 1.04 & $5.13 \mathrm{E}-08$ & $5.23 \mathrm{E}-08$ & 4.99 & $1.02 \mathrm{E}-08$ & $1.19 \mathrm{E}-08$ \\
\hline 1.08 & $5.04 \mathrm{E}-08$ & $5.14 E-08$ & 5.15 & $7.12 \mathrm{E}-09$ & $8.98 \mathrm{E}-09$ \\
\hline 1.13 & $5.14 \mathrm{E}-08$ & $5.24 \mathrm{E}-08$ & 5.32 & $3.41 \mathrm{E}-09$ & $5.28 \mathrm{E}-09$ \\
\hline 1.17 & $5.25 \mathrm{E}-08$ & $5.35 \mathrm{E}-08$ & 5.50 & $2.19 \mathrm{E}-09$ & $4.26 \mathrm{E}-09$ \\
\hline 1.22 & $5.23 E-08$ & $5.36 \mathrm{E}-08$ & 5.67 & $5.17 \mathrm{E}-09$ & $7.35 \mathrm{E}-09$ \\
\hline 1.28 & $5.12 E-08$ & $5.25 \mathrm{E}-08$ & 5.83 & $1.05 \mathrm{E}-08$ & $1.26 \mathrm{E}-08$ \\
\hline 1.33 & $4.95 \mathrm{E}-08$ & $5.06 \mathrm{E}-08$ & 6.00 & $1.48 \mathrm{E}-08$ & $1.67 \mathrm{E}-08$ \\
\hline 1.38 & $4.72 \mathrm{E}-08$ & $4.82 \mathrm{E}-08$ & 6.18 & $1.59 \mathrm{E}-08$ & $1.77 \mathrm{E}-08$ \\
\hline 1.42 & $4.45 \mathrm{E}-08$ & $4.55 \mathrm{E}-08$ & 6.35 & $1.39 \mathrm{E}-08$ & $1.57 \mathrm{E}-08$ \\
\hline 1.47 & $4.14 E-08$ & $4.23 \mathrm{E}-08$ & 6.51 & $1.07 \mathrm{E}-08$ & $1.23 \mathrm{E}-08$ \\
\hline 1.53 & $3.84 \mathrm{E}-08$ & $3.93 \mathrm{E}-08$ & 6.70 & $8.14 \mathrm{E}-09$ & $9.43 \mathrm{E}-09$ \\
\hline 1.58 & $3.63 \mathrm{E}-08$ & $3.72 \mathrm{E}-08$ & 6.90 & $7.41 \mathrm{E}-09$ & $8.56 \mathrm{E}-09$ \\
\hline $\begin{array}{l}1.64 \\
1.72 \\
1.80\end{array}$ & $\begin{array}{l}3.54 \mathrm{E}-08 \\
3.66 \mathrm{E}-08 \\
3.94 \mathrm{E}-08\end{array}$ & $\begin{array}{l}3.63 \mathrm{E}-08 \\
3.75 \mathrm{E}-08 \\
4.03 \mathrm{E}-08\end{array}$ & $\begin{array}{l}7.10 \\
7.30 \\
7.50\end{array}$ & $\begin{array}{l}8.39 \mathrm{E}-09 \\
9.67 \mathrm{E}-09 \\
9.92 \mathrm{E}-09\end{array}$ & $\begin{array}{l}9.52 \mathrm{E}-09 \\
1.07 \mathrm{E}-08 \\
1.09 \mathrm{E}-08\end{array}$ \\
\hline 1.88 & $4.28 \mathrm{E}-08$ & $4.36 \mathrm{E}-08$ & 7.70 & $8.75 \mathrm{E}-09$ & $9.65 E-09$ \\
\hline 1.96 & $4.51 E-08$ & $4.60 \mathrm{E}-08$ & 7.90 & $6.63 \mathrm{E}-09$ & $7.44 \mathrm{E}-09$ \\
\hline 2.04 & $4.61 \mathrm{E}-08$ & $4.70 E-08$ & 8.10 & $4.37 \mathrm{E}-09$ & $5.09 \mathrm{E}-09$ \\
\hline 2.12 & $4.60 \mathrm{E}-08$ & $4.68 \mathrm{E}-08$ & 8.30 & $2.54 \mathrm{E}-09$ & $3.18 \mathrm{E}-09$ \\
\hline 2.20 & $4.42 \mathrm{E}-08$ & $4.50 \mathrm{E}-08$ & 8.50 & $1.46 \mathrm{E}-09$ & $1.99 \mathrm{E}-09$ \\
\hline 2.28 & $4.00 \mathrm{E}-08$ & $4.07 \mathrm{E}-08$ & 8.73 & $9.20 \mathrm{E}-10$ & $1.39 \mathrm{E}-09$ \\
\hline 2.36 & $3.37 \mathrm{E}-08$ & $3.44 \mathrm{E}-08$ & 8.98 & $7.98 \mathrm{E}-10$ & $1.21 \mathrm{E}-09$ \\
\hline 2.45 & $2.62 \mathrm{E}-08$ & $2.69 \mathrm{E}-08$ & 9.23 & $7.31 \mathrm{E}-10$ & $1.08 \mathrm{E}-09$ \\
\hline 2.55 & $2.00 \mathrm{E}-08$ & $2.07 E-08$ & 9.48 & $5.28 \mathrm{E}-10$ & $8.31 \mathrm{E}-10$ \\
\hline 2.65 & $1.72 \mathrm{E}-08$ & $1.78 \mathrm{E}-08$ & 9.73 & $2.3 \cdot 8 \mathrm{E}-10$ & $5.04 \mathrm{E}-10$ \\
\hline 2.75 & $1.63 \mathrm{E}-08$ & $1.70 \mathrm{E}-08$ & 9.98 & $-4.26 \mathrm{E}-11$ & $2.04 \mathrm{E}-10$ \\
\hline 2.85 & $1.57 \mathrm{E}-08$ & $1.63 \mathrm{E}-08$ & 10.30 & $-2.45 \mathrm{E}-10$ & $2.46 \mathrm{E}-13$ \\
\hline & $1.43 \mathrm{E}-08$ & $1.49 \mathrm{E}-08$ & 10.70 & $-2.82 \mathrm{E}-10$ & $1.02 \mathrm{E}-11$ \\
\hline 3.05 & $1.28 \mathrm{E}-08$ & $1.34 E-08$ & 11.10 & $-2.37 \mathrm{E}-10$ & $9.07 \mathrm{E}-11$ \\
\hline 3.15 & $1.18 \mathrm{E}-08$ & $1.24 \mathrm{E}-08$ & 11.50 & $-1.96 \mathrm{E}-10$ & $1.35 \mathrm{E}-10$ \\
\hline 3.25 & $1.15 \mathrm{E}-08$ & $1.22 \mathrm{E}-08$ & 11.90 & $-1.39 \mathrm{E}-10$ & $1.63 \mathrm{E}-10$ \\
\hline 3.35 & $1.18 \mathrm{E}-08$ & $1.24 \mathrm{E}-08$ & 12.30 & $-4.44 \mathrm{E}-11$ & $1.88 \mathrm{E}-10$ \\
\hline 3.45 & $1.21 \mathrm{E}-08$ & $1.27 \mathrm{E}-08$ & 12.70 & $7.03 \mathrm{E}-11$ & $2.15 \mathrm{E}-10$ \\
\hline 3.55 & 1. $23 \mathrm{E}-08$ & $1.30 \mathrm{E}-08$ & 13.10 & $1.38 \mathrm{E}-10$ & $2.65 \mathrm{E}-10$ \\
\hline 3.66 & $1.23 \mathrm{E}-08$ & $1.30 \mathrm{E}-08$ & 13.50 & $1.10 \mathrm{E}-10$ & $2.96 \mathrm{E}-10$ \\
\hline 3.79 & $1.17 E-08$ & $1.25 \mathrm{E}-08$ & 13.90 & $7.30 \mathrm{E}-11$ & $2.70 \mathrm{E}-10$ \\
\hline
\end{tabular}


Table. A.13

Neutron Data

Detector 13 in Fig. 4

Detector Coordinates $(\mathrm{cm}): x=0.0, y=0.0, z^{\prime}=181.0$

\begin{tabular}{|c|c|c|c|c|c|}
\hline $\begin{array}{l}\text { Energy } \\
(\mathrm{MeV})\end{array}$ & \multicolumn{2}{|c|}{$\underline{F} 1 \underline{\mathrm{u}} \mathrm{e} \underline{\mathrm{n}} \mathrm{c} \underline{\mathrm{e}}-\left(\mathrm{Me} \mathrm{V}^{-1} \mathrm{c} \underline{m}^{-2}\right)$} & $\begin{array}{l}\text { Energy } \\
(\mathrm{MeV})\end{array}$ & \multicolumn{2}{|c|}{ 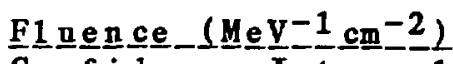 } \\
\hline 0.91 & $6.93 \mathrm{E}-07$ & $7.02 \mathrm{E}-07$ & 5.94 & $4.13 \mathrm{E}-08$ & $4.38 \mathrm{E}-08$ \\
\hline 1.01 & $6.78 \mathrm{E}-07$ & $6.85 \mathrm{E}-07$ & 6.25 & $3.51 \mathrm{E}-08$ & $3.81 \mathrm{E}-08$ \\
\hline 1.11 & $5.60 \mathrm{E}-07$ & $5.69 \mathrm{E}-07$ & 6.55 & $3.24 \mathrm{E}-08$ & $3.56 \mathrm{E}-08$ \\
\hline 1.21 & $5.01 \mathrm{E}-07$ & $5.10 \mathrm{E}-07$ & 6.83 & $3.33 \mathrm{E}-08$ & $3.61 \mathrm{E}-08$ \\
\hline 1.31 & $5.42 \mathrm{E}-07$ & $5.49 \mathrm{E}-07$ & 7.23 & $3.25 \mathrm{E}-08$ & $3.51 \mathrm{E}-08$ \\
\hline 1.41 & $6.15 \mathrm{E}-07$ & $6.22 \mathrm{E}-07$ & 7.73 & $2.13 \mathrm{E}-08$ & $2.43 \mathrm{E}-08$ \\
\hline 1.51 & $6.25 \mathrm{E}-07$ & $6.33 \mathrm{E}-07$ & 8.25 & $2.24 E-09$ & $5.06 \mathrm{E}-09$ \\
\hline 1.61 & $5.53 \mathrm{E}-07$ & $5.60 \mathrm{E}-07$ & 8.77 & $1.55 \mathrm{E}-09$ & $4.08 \mathrm{E}-09$ \\
\hline 1.71 & $4.36 \mathrm{E}-07$ & $4.41 \mathrm{E}-07$ & 9.24 & $5.97 \mathrm{E}-09$ & $8.71 \mathrm{E}-09$ \\
\hline 1.81 & $3.26 \mathrm{E}-07$ & 3.32 E- 07 & 9.72 & $3.87 \mathrm{E}-09$ & $6.41 \mathrm{E}-09$ \\
\hline 1.94 & $2.49 \mathrm{E}-07$ & $2.55 \mathrm{E}-07$ & 10.23 & $6.89 \mathrm{E}-09$ & $9.57 \mathrm{E}-09$ \\
\hline 2.11 & $2.48 \mathrm{E}-07$ & $2.52 \mathrm{E}-07$ & 10.75 & $1.76 \mathrm{E}-08$ & $2.06 \mathrm{E}-08$ \\
\hline 2.31 & $2.43 \mathrm{E}-07$ & $2.48 \mathrm{E}-07$ & 11.26 & $2.19 \mathrm{E}-08$ & $2.49 \mathrm{E}-08$ \\
\hline 2.50 & $1.73 \mathrm{E}-07$ & $1.76 \mathrm{E}-07$ & 11.76 & $1.98 \mathrm{E}-08$ & $2.28 \mathrm{E}-08$ \\
\hline 2.69 & $1.25 \mathrm{E}-07$ & $1.29 \mathrm{E}-07$ & 12.40 & $5.44 \mathrm{E}-08$ & 5.75 E- 08 \\
\hline 2.90 & $1.55 \mathrm{E}-07$ & $1.59 \mathrm{E}-07$ & 13.20 & $2.69 \mathrm{E}-07$ & $2.72 \mathrm{E}-07$ \\
\hline 3.10 & $1.84 \mathrm{E}-07$ & $1.88 \mathrm{E}-07$ & 14.03 & $6.31 \mathrm{E}-07$ & $6.33 \mathrm{E}-07$ \\
\hline & $1.56 \mathrm{E}-07$ & $1.59 \mathrm{E}-07$ & 14.86 & $8.06 \mathrm{E}-07$ & $8.08 \mathrm{E}-07$ \\
\hline 3.51 & $1.07 \mathrm{E}-07$ & $1.11 \mathrm{E}-07$ & 15.62 & $6.77 \mathrm{E}-07$ & $6.79 E-07$ \\
\hline 3.71 & $8.49 \mathrm{E}-08$ & $8.96 \mathrm{E}-08$ & 16.45 & $3.96 \mathrm{E}-07$ & $3.97 \mathrm{E}-07$ \\
\hline 3.90 & $8.44 \mathrm{E}-08$ & $8.76 \mathrm{E}-08$ & 17.44 & $1.49 \mathrm{E}-07$ & $1.50 \mathrm{E}-07$ \\
\hline 4.15 & $7.96 \mathrm{E}-08$ & $8.32 \mathrm{E}-08$ & 18.51 & $3.77 E-08$ & $3.79 \mathrm{E}-08$ \\
\hline 4.46 & $6.45 \mathrm{E}-08$ & $6.67 \mathrm{E}-08$ & 19.58 & $7.11 \mathrm{E}-09$ & $7.20 \mathrm{E}-09$ \\
\hline 4.75 & $5.46 \mathrm{E}-08$ & $5.72 \mathrm{E}-08$ & 20.53 & $4.44 E-10$ & $4.91 \mathrm{E}-10$ \\
\hline 5.06 & $4.77 \mathrm{E}-08$ & $5.08 \mathrm{E}-08$ & 21.43 & $-1.36 E-09$ & $-1.33 \mathrm{E}-09$ \\
\hline 5.36 & $4.26 \mathrm{E}-08$ & $4.59 \mathrm{E}-08$ & 22.40 & $-1.88 \mathrm{E}-09$ & $-1.84 E-09$ \\
\hline 5.64 & $4.27 \mathrm{E}-08$ & $4.56 \mathrm{E}-08$ & 23.43 & $-1.70 \mathrm{E}-09$ & $-1.67 \mathrm{E}-09$ \\
\hline
\end{tabular}


Table A.13 (Cont ' $d$ )

Gamma-Ray Data

Detector 13 in Fig. 4

Detector Coordinates $(\mathrm{cm}): \quad x=0.0, y=0.0, z^{\prime}=181.0$

\begin{tabular}{|c|c|c|c|c|c|}
\hline \multirow{2}{*}{$\begin{array}{l}\text { Energy } \\
(\mathrm{MeV})\end{array}$} & \multicolumn{2}{|c|}{$\underline{F} \underline{1} \underline{\underline{z}} \underline{\mathrm{e}} \mathrm{n} \underline{\mathrm{c}}-\left(\mathrm{Me}-\mathrm{V}^{-1} \mathrm{~cm}^{-2}\right)$} & \multirow{2}{*}{$\begin{array}{l}\text { Energy } \\
\text { (MeV) }\end{array}$} & \multicolumn{2}{|c|}{ 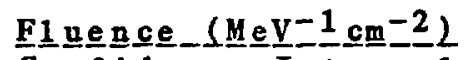 } \\
\hline & Conf idence & Interva & & Confidence & Interval \\
\hline 0.72 & $8.16 \mathrm{E}-07$ & $1.37 \mathrm{E}-06$ & 3.93 & $1.11 \mathrm{E}-07$ & $1.19 \mathrm{E}-07$ \\
\hline 0.76 & $1.50 \mathrm{E}-06$ & $1.73 \mathrm{E}-06$ & 4.06 & $9.53 \mathrm{E}-08$ & $1.04 \mathrm{E}-07$ \\
\hline 0.80 & $1.96 \mathrm{E}-06$ & $2.03 \mathrm{E}-06$ & 4.19 & $8.83 \mathrm{E}-08$ & $9.76 \mathrm{E}-08$ \\
\hline 0.84 & $2.09 \mathrm{E}-06$ & $2.11 \mathrm{E}-06$ & 4.31 & $9.64 \mathrm{E}-08$ & $1.07 \mathrm{E}-07$ \\
\hline 0.88 & $1.91 E-06$ & $1.93 \mathrm{E}-06$ & 4.44 & $1.21 \mathrm{E}-07$ & $1.34 \mathrm{E}-07$ \\
\hline 0.92 & $1.59 \mathrm{E}-06$ & $1.61 \mathrm{E}-06$ & 4.57 & $1.51 \mathrm{E}-07$ & $1.65 \mathrm{E}-07$ \\
\hline 0.96 & $1.27 \mathrm{E}-06$ & $1.29 \mathrm{E}-06$ & 4.70 & $1.68 \mathrm{E}-07$ & $1.82 \mathrm{E}-07$ \\
\hline 1.00 & $1.03 \mathrm{E}-06$ & $1.05 \mathrm{E}-06$ & 4.83 & $1.57 \mathrm{E}-07$ & $1.71 \mathrm{E}-07$ \\
\hline 1.04 & $8.93 \mathrm{E}-07$ & $9.07 \mathrm{E}-07$ & 4.99 & $1.17 \mathrm{E}-07$ & $1.34 \mathrm{E}-07$ \\
\hline 1.08 & $8.40 \mathrm{E}-07$ & $8.53 \mathrm{E}-07$ & 5.15 & $7.17 \mathrm{E}-08$ & $8.97 \mathrm{E}-08$ \\
\hline 1.13 & $8.62 \mathrm{E}-07$ & $8.74 E-07$ & 5.32 & $5.90 \mathrm{E}-08$ & $7.81 \mathrm{E}-08$ \\
\hline 1.17 & $9.36 \mathrm{E}-07$ & $9.48 \mathrm{E}-07$ & 5.50 & $6.86 \mathrm{E}-08$ & $8.88 E-08$ \\
\hline 1.22 & $9.93 \mathrm{E}-07$ & $1.01 \mathrm{E}-06$ & 5.67 & $7.59 \mathrm{E}-08$ & $9.77 \mathrm{E}-08$ \\
\hline 1.28 & $9.88 \mathrm{E}-07$ & $1.00 \mathrm{E}-06$ & & $7.10 \mathrm{E}-08$ & $9.18 \mathrm{E}-08$ \\
\hline 1.33 & $9.19 \mathrm{E}-07$ & $9.30 \mathrm{E}-0.7$ & 6.00 & $6.54 \mathrm{E}-08$ & $8.43 \mathrm{E}-08$ \\
\hline 1.38 & $8.07 \mathrm{E}-07$ & $8.18 \mathrm{E}-07$ & 6.18 & $6.94 \mathrm{E}-08$ & $8.81 \mathrm{E}-08$ \\
\hline 1.42 & $6.85 \mathrm{E}-07$ & $6.96 \mathrm{E}-07$ & 6.35 & $8.06 \mathrm{E}-08$ & $9.86 \mathrm{E}-08$ \\
\hline 1.47 & $5.75 \mathrm{E}-07$ & $5.85 \mathrm{E}-07$ & 6.51 & $8.89 \mathrm{E}-08$ & $1.05 \mathrm{E}-07$ \\
\hline 1.53 & $4.90 E-07$ & $5.00 \mathrm{E}-07$ & 6.70 & $8.34 \mathrm{E}-08$ & $9.68 \mathrm{E}-08$ \\
\hline 1.58 & $4.35 \mathrm{E}-07$ & $4.45 \mathrm{E}-07$ & 6.90 & $6.40 \mathrm{E}-08$ & $7.61 \mathrm{E}-08$ \\
\hline 1.64 & $4.07 \mathrm{E}-07$ & $4.17 \mathrm{E}-07$ & 7.10 & $4.63 \mathrm{E}-08$ & $5.83 \mathrm{E}-08$ \\
\hline 1.72 & $4.09 \mathrm{E}-07$ & $4.18 \mathrm{E}-07$ & 7.30 & $4.15 \mathrm{E}-08$ & $5.28 \mathrm{E}-08$ \\
\hline 1.80 & $4.15 \mathrm{E}-07$ & $4.24 \mathrm{E}-07$ & 7.50 & $4.76 \mathrm{E}-08$ & $5.83 \mathrm{E}-08$ \\
\hline 1.88 & $4.08 \mathrm{E}-07$ & $4.17 \mathrm{E}-07$ & 7.70 & $5.45 \mathrm{E}-08$ & $6.47 \mathrm{E}-08$ \\
\hline 1.96 & $3.82 \mathrm{E}-07$ & $3.91 \mathrm{E}-07$ & 7.90 & $5.46 \mathrm{E}-08$ & $6.43 \mathrm{E}-0.8$ \\
\hline 2.04 & $3.47 \mathrm{E}-07$ & $3.55 \mathrm{E}-07$ & 8.10 & $4.61 \mathrm{E}-08$ & $5.52 \mathrm{E}-08$ \\
\hline 2.12 & $3.20 \mathrm{E}-07$ & $3.28 \mathrm{E}-07$ & 8.30 & $3.30 \mathrm{E}-08$ & $4.13 \mathrm{E}-08$ \\
\hline 2.20 & $3.05 \mathrm{E}-07$ & $3.13 \mathrm{E}-07$ & 8.50 & $2.15 \mathrm{E}-08$ & $2.93 \mathrm{E}-08$ \\
\hline 2.28 & $2.85 \mathrm{E}-07$ & $2.93 \mathrm{E}-07$ & 8.73 & $1.48 \mathrm{E}-08$ & $2.19 \mathrm{E}-08$ \\
\hline 2.36 & $2.56 \mathrm{E}-07$ & $2.64 E-07$ & 8.98 & $1.56 \mathrm{E}-08$ & $2.18 \mathrm{E}-08$ \\
\hline 2.45 & $2.30 \mathrm{E}-07$ & $2.37 \mathrm{E}-07$ & 9.23 & $1.96 \mathrm{E}-08$ & $2.54 \mathrm{E}-08$ \\
\hline 2.55 & $2.20 \mathrm{E}-07$ & $2.27 \mathrm{E}-07$ & 9.48 & $2.14 \mathrm{E}-08$ & $2.66 \mathrm{E}-08$ \\
\hline 2.65 & $2.13 \mathrm{E}-07$ & $2.20 \mathrm{E}-07$ & 9.73 & $1.82 \mathrm{E}-08$ & $2.27 \mathrm{E}-08$ \\
\hline 2.75 & $1.99 \mathrm{E}-07$ & $2.05 \mathrm{E}-07$ & 9.98 & $1.10 \mathrm{E}-08$ & $1.52 \mathrm{E}-0.8$ \\
\hline 2.85 & $1.82 \mathrm{E}-07$ & $1.89 \mathrm{E}-07$ & 10.30 & $9.34 \mathrm{E}-10$ & $4.44 E-09$ \\
\hline 2.95 & $1.70 \mathrm{E}-07$ & $1.77 \mathrm{E}-07$ & 10.70 & $-7.70 \mathrm{E}-09$ & $-4.28 E-09$ \\
\hline 3.05 & $1.63 \mathrm{E}-07$ & $1.70 \mathrm{E}-07$ & 11.10 & $-1.15 \mathrm{E}-08$ & $-7.49 \mathrm{E}-09$ \\
\hline 3.15 & $1.59 \mathrm{E}-07$ & $1.66 \mathrm{E}-07$ & 11.50 & $-1.22 \mathrm{E}-08$ & $-7.79 \mathrm{E}-09$ \\
\hline 3.25 & $1.58 \mathrm{E}-07$ & $1.65 \mathrm{E}-07$ & 11.90 & $-1.07 \mathrm{E}-08$ & $-6.53 \mathrm{E}-09$ \\
\hline 3.35 & $1.58 \mathrm{E}-07$ & $1.65 \mathrm{E}-07$ & 12.30 & $-7.09 \mathrm{E}-09$ & $-3.79 \mathrm{E}-09$ \\
\hline 3.45 & $1.57 \mathrm{E}-07$ & $1.64 \mathrm{E}-07$ & 12.70 & $-1.76 \mathrm{E}-09$ & $2.56 \mathrm{E}-10$ \\
\hline 3.55 & $1.53 \mathrm{E}-07$ & $1.61 \mathrm{E}-07$ & 13.10 & $3.50 \mathrm{E}-09$ & $4.71 \mathrm{E}-09$ \\
\hline 3.66 & $1.44 \mathrm{E}-07$ & $1.52 \mathrm{E}-07$ & 13.50 & $6.54 \mathrm{E}-09$ & $8.57 E-091$ \\
\hline & $1.29 \mathrm{E}-07$ & $1.37 \mathrm{E}-07$ & 13.90 & $7.25 \mathrm{E}-09$ & $9.76 \mathrm{E}-09$ \\
\hline & & , $\quad$ sll & & & \\
\hline
\end{tabular}


Table A.14

\section{Neutron Data}

Detector 14 in Fig. 4

Detector Coordinates $(\mathrm{cm}): x=81.0, y=0.0, z^{\prime}=181.0$

\begin{tabular}{|c|c|c|c|c|c|}
\hline $\begin{array}{l}\text { Energy } \\
(\mathrm{MeV})\end{array}$ & 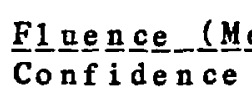 & 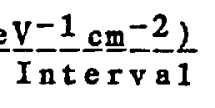 & $\begin{array}{l}\text { Energy } \\
(\mathrm{MeV})\end{array}$ & 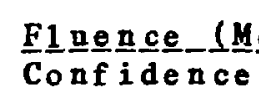 & $\left.=\frac{V-1}{I n t e r} m^{-2}\right)$ \\
\hline 0.91 & $1.31 \mathrm{E}-07$ & $1.33 \mathrm{E}-07$ & 5.94 & $3.82 \mathrm{E}-09$ & $4.03 \mathrm{E}-09$ \\
\hline 1.01 & $1.23 \mathrm{E}-07$ & $1.24 \mathrm{E}-07$ & 6.25 & $3.53 \mathrm{E}-09$ & $3.76 \mathrm{E}-09$ \\
\hline 1.11 & $1.11 \mathrm{E}-07$ & $1.12 \mathrm{E}-07$ & 6.55 & $3.31 \mathrm{E}-09$ & $3.55 \mathrm{E}-09$ \\
\hline 1.21 & $9.88 \mathrm{E}-08$ & $9.97 \mathrm{E}-08$ & 6.83 & $3.10 \mathrm{E}-09$ & $3.30 \mathrm{E}-09$ \\
\hline 1.31 & $8.82 \mathrm{E}-08$ & $8.89 \mathrm{E}-08$ & 7.23 & $2.70 \mathrm{E}-09$ & $2.88 \mathrm{E}-0.9$ \\
\hline 1.41 & $7.92 \mathrm{E}-08$ & $8.00 \mathrm{E}-08$ & 7.73 & $2.16 \mathrm{E}-09$ & $2.38 \mathrm{E}-09$ \\
\hline 1.51 & $7.20 \mathrm{E}-08$ & $7.28 \mathrm{E}-08$ & 8.25 & $1.74 \mathrm{E}-09$ & $1.93 \mathrm{E}-09$ \\
\hline 1.61 & $6.44 \mathrm{E}-08$ & $6.51 \mathrm{E}-08$ & 8.77 & $1.53 \mathrm{E}-09$ & $1.68 \mathrm{E}-09$ \\
\hline 1.71 & $5.67 E-08$ & $5.73 \mathrm{E}-08$ & 9.24 & $1.37 \mathrm{E}-09$ & $1.54 \mathrm{E}-09$ \\
\hline 1.81 & $5.00 \mathrm{E}-08$ & $5.07 \mathrm{E}-08$ & 9.72 & $1.33 \mathrm{E}-09$ & $1.47 \mathrm{E}-09$ \\
\hline 1.94 & $4.41 \mathrm{E}-08$ & $4.49 E-08$ & 10.23 & $1.47 \mathrm{E}-09$ & $1.62 \mathrm{E}-09$ \\
\hline 2.11 & $4.01 \mathrm{E}-08$ & $4.06 \mathrm{E}-08$ & 10.75 & $1.59 \mathrm{E}-09$ & $1.75 E-09$ \\
\hline 2.31 & $3.50 \mathrm{E}-08$ & $3.56 \mathrm{E}-08$ & 11.26 & $1.50 \mathrm{E}-09$ & $1.66 \mathrm{E}-09$ \\
\hline 2.50 & $2.91 \mathrm{E}-08$ & $2.95 \mathrm{E}-08$ & 11.76 & $1.45 \mathrm{E}-09$ & $1.61 \mathrm{E}-09$ \\
\hline 2.69 & $2.42 \mathrm{E}-08$ & $2.45 \mathrm{E}-08$ & 12.40 & $2.10 \mathrm{E}-09$ & $2.25 \mathrm{E}-09$ \\
\hline 2.90 & $2.04 \mathrm{E}-08$ & $2.07 \mathrm{E}-08$ & 13.20 & $4.42 \mathrm{E}-09$ & $4.55 \mathrm{E}-09$ \\
\hline 3.10 & $1.67 \mathrm{E}-08$ & $1.72 \mathrm{E}-08$ & 14.03 & $7.22 \mathrm{E}-09$ & $7.34 \mathrm{E}-09$ \\
\hline 3.30 & $1.34 E-08$ & $1.38 E-08$ & 14.86 & $7.65 \mathrm{E}-09$ & $7.75 \mathrm{E}-09$ \\
\hline 3.51 & $1.09 E-08$ & $1.13 \mathrm{E}-08$ & 15.62 & $5.66 \mathrm{E}-09$ & $5.74 \mathrm{E}-09$ \\
\hline $\begin{array}{l}3.71 \\
3.90 \\
4.15\end{array}$ & $\begin{array}{l}9.49 \mathrm{E}-09 \\
8.83 \mathrm{E}-09 \\
7.98 \mathrm{E}-09\end{array}$ & $\begin{array}{l}9.94 \mathrm{E}-09 \\
9.14 \mathrm{E}-09 \\
8.32 \mathrm{E}-09\end{array}$ & $\begin{array}{l}16.45 \\
17.44 \\
18.51\end{array}$ & $\begin{array}{l}2.95 \mathrm{E}-09 \\
9.93 \mathrm{E}-10 \\
2.23 \mathrm{E}-10\end{array}$ & $\begin{array}{l}3.00 \mathrm{E}-09 \\
1.02 \mathrm{E}-09 \\
2.36 \mathrm{E}-10\end{array}$ \\
\hline 4.46 & $6.85 \mathrm{E}-09$ & $7.04 \mathrm{E}-09$ & 19.58 & $3.12 \mathrm{E}-11$ & $4.21 \mathrm{E}-11$ \\
\hline 4.75 & $5.84 \mathrm{E}-09$ & $6.07 \mathrm{E}-09$ & 20.53 & $-8.26 \mathrm{E}-12$ & $1.41 \mathrm{E}-12$ \\
\hline 5.06 & $5.01 \mathrm{E}-09$ & $5.26 \mathrm{E}-09$ & 21.43 & -1.85 E-11 & $-1.06 \mathrm{E}-11$ \\
\hline 5.36 & $4.38 \mathrm{E}-09$ & $4.65 \mathrm{E}-09$ & 22.40 & $-2.25 \mathrm{E}-11$ & $-1.39 \mathrm{E}-11$ \\
\hline 5.64 & $4.07 E-09$ & $4.30 \mathrm{E}-09$ & 23.43 & $-2.05 E-11$ & $-1.11 \mathrm{E}-11$ \\
\hline
\end{tabular}


Table A.14 (Cont'd)

Gamma-Ray Data

Detector 14 in Fig. 4

Detector Coordinates $(\mathrm{cm}): x=81.0, y=0.0, z^{\prime}=181.0$

\begin{tabular}{|c|c|c|c|c|c|}
\hline $\begin{array}{l}\text { Energg } \\
(\mathrm{MeV})\end{array}$ & $\frac{F}{\text { Conf }}-\underline{\text { ence }}$ idence & $\frac{V-1}{\text { Interva }-\frac{2}{c} L}$ & $\begin{array}{l}\text { Energy } \\
(\mathrm{MeV})\end{array}$ & $\frac{\text { Fin }}{\text { Conf }}$ idence & $=\frac{v-1}{I n t e r m-2}=\frac{1}{1}$ \\
\hline 0.72 & $1.31 \overline{1}-0 \overline{7}$ & $2.16 \mathrm{E}-07$ & 3.93 & $2.29 \mathrm{E}-08$ & $2.46 \mathrm{E}-08$ \\
\hline 0.76 & $2.02 E-07$ & $2.36 \mathrm{E}-07$ & 4.06 & $2.07 \mathrm{E}-08$ & $2.26 \mathrm{E}-08$ \\
\hline 0.80 & $2.39 \mathrm{E}-07$ & $2.49 \mathrm{E}-07$ & 4.19 & $1.99 \mathrm{E}-08$ & $2.19 \mathrm{E}-08$ \\
\hline 0.84 & $2.43 \mathrm{E}-07$ & $2.47 \mathrm{E}-07$ & 4.31 & $2.00 \mathrm{E}-08$ & $2.24 \mathrm{E}-08$ \\
\hline 0.88 & $2.23 E-07$ & $2.26 \mathrm{E}-07$ & 4.44 & $2.02 \mathrm{E}-08$ & $2.30 \mathrm{E}-08$ \\
\hline 0.92 & $1.94 E-07$ & $1.97 \mathrm{E}-07$ & 4.57 & $2.01 \mathrm{E}-08$ & $2.31 \mathrm{E}-08$ \\
\hline 0.96 & $1.65 \mathrm{E}-07$ & $1.68 \mathrm{E}-07$ & 4.70 & $1.96 \mathrm{E}-08$ & $2.26 \mathrm{E}-08$ \\
\hline 1.00 & $1.43 E-07$ & $1.46 E-07$ & 4.83 & $1.85 \mathrm{E}-08$ & $2.17 \mathrm{E}-0.8$ \\
\hline 1.04 & $1.29 \mathrm{E}-07$ & $1.32 \mathrm{E}-07$ & 4.99 & $1.68 \mathrm{E}-08$ & $2.06 \mathrm{E}-08$ \\
\hline 1.08 & $1.23 \mathrm{E}-07$ & $1.25 \mathrm{E}-07$ & 5.15 & $1.49 \mathrm{E}-08$ & $1.90 \mathrm{E}-08$ \\
\hline 1.13 & $1.21 E-07$ & $1.23 \mathrm{E}-07$ & 5.32 & $1.35 \mathrm{E}-08$ & $1.76 \mathrm{E}-08$ \\
\hline 1.17 & $1.22 \mathrm{E}-07$ & $1.24 \mathrm{E}-07$ & 5.50 & $1.31 \mathrm{E}-08$ & $1.76 \mathrm{E}-08$ \\
\hline 1.22 & $1.21 \mathrm{E}-07$ & $1.24 \mathrm{E}-07$ & 5.67 & $1.50 \mathrm{E}-08$ & $1.98 \mathrm{E}-08$ \\
\hline 1.28 & $1.19 \mathrm{E}-07$ & $1.22 \mathrm{E}-07$ & 5.83 & $1.93 \mathrm{E}-08$ & $2.38 \mathrm{E}-08$ \\
\hline 1.33 & $1.15 \mathrm{E}-07$ & $1.17 \mathrm{E}-07$ & 6.00 & $2.40 \mathrm{E}-08$ & $2.81 \mathrm{E}-08$ \\
\hline 1.38 & $1.10 E-07$ & $1.12 \mathrm{E}-07$ & 6.18 & $2.70 \mathrm{E}-08$ & $3.12 \mathrm{E}-08$ \\
\hline 1.42 & $1.02 E-07$ & $1.04 \mathrm{E}-07$ & 6.35 & $2.74 \mathrm{E}-08$ & $3.13 \mathrm{E}-08$ \\
\hline 1.47 & $9.24 E-08$ & $9.45 \mathrm{E}-08$ & 6.51 & $2.56 \mathrm{E}-08$ & $2.90 \mathrm{E}-08$ \\
\hline 1.53 & $8.46 \mathrm{E}-08$ & $8.66 \mathrm{E}-08$ & 6.70 & $2.23 \mathrm{E}-08$ & $2.52 \mathrm{E}-08$ \\
\hline 1.58 & $7.99 \mathrm{E}-08$ & $8.19 \mathrm{E}-08$ & 6.90 & $1.90 \mathrm{E}-08$ & $2.15 \mathrm{E}-08$ \\
\hline $\begin{array}{l}1.64 \\
1.72 \\
1.80\end{array}$ & $\begin{array}{l}7.83 \mathrm{E}-08 \\
8.08 \mathrm{E}-08 \\
8.63 \mathrm{E}-08\end{array}$ & $\begin{array}{l}8.02 E-08 \\
8.28 E-08 \\
8.84 E-08\end{array}$ & $\begin{array}{l}7.10 \\
7.30 \\
7.50\end{array}$ & $\begin{array}{l}1.72 \mathrm{E}-08 \\
1.72 \mathrm{E}-08 \\
1.76 \mathrm{E}-08\end{array}$ & $\begin{array}{l}1.97 \mathrm{E}-08 \\
1.94 \mathrm{E}-08 \\
1.98 \mathrm{E}-08\end{array}$ \\
\hline 1.88 & $9.16 \mathrm{E}-08$ & $9.36 \mathrm{E}-08$ & 7.70 & $1.71 \mathrm{E}-08$ & $1.90 \mathrm{E}-08$ \\
\hline $\begin{array}{l}1.96 \\
2.04\end{array}$ & $\begin{array}{l}9.37 \mathrm{E}-08 \\
9.34 \mathrm{E}-08\end{array}$ & $\begin{array}{l}9.57 \mathrm{E}-08 \\
9.52 \mathrm{E}-08\end{array}$ & $\begin{array}{l}7.90 \\
8.10\end{array}$ & $\begin{array}{l}1.47 \mathrm{E}-08 \\
10 \mathrm{R}-08\end{array}$ & $\begin{array}{l}1.64 \mathrm{E}-08 \\
25 \mathrm{E}-08\end{array}$ \\
\hline 2.12 & $9.27 \mathrm{E}-08$ & $9.44 \mathrm{E}-08$ & 8.30 & $6.93 \mathrm{E}-09$ & $8.25 \mathrm{E}-09$ \\
\hline 2.20 & $9.00 \mathrm{E}-08$ & $9.17 \mathrm{E}-08$ & 8.50 & $3.69 \mathrm{E}-09$ & $4.85 \mathrm{E}-09$ \\
\hline 2.28 & $8.22 \mathrm{E}-08$ & $8.38 \mathrm{E}-08$ & 8.73 & $1.59 \mathrm{E}-09$ & $2.62 \mathrm{E}-09$ \\
\hline 2.36 & $6.92 \mathrm{E}-08$ & $7.08 \mathrm{E}-08$ & 8.98 & $9.83 \mathrm{E}-10$ & $1.90 \mathrm{E}-09$ \\
\hline 2.45 & $5.41 \mathrm{E}-08$ & $5.57 \cdot \mathrm{E}-08$ & 9.23 & $1.22 \mathrm{E}-09$ & $1.97 \mathrm{E}-09$ \\
\hline 2.55 & $4.34 \mathrm{E}-08$ & $4.49 \mathrm{E}-08$ & & & $2.06 \mathrm{E}-09$ \\
\hline 2.65 & $3.90 \mathrm{E}-08$ & $4.05 E-08$ & 9.73 & $1.17 \mathrm{E}-09$ & $1.84 E-09$ \\
\hline 2.75 & $3.68 \mathrm{E}-08$ & $3.83 \mathrm{E}-08$ & 9.98 & $6.35 \mathrm{E}-10$ & $1.41 \mathrm{E}-09$ \\
\hline 2.85 & $3.43 \mathrm{E}-08$ & $3.57 \mathrm{E}-08$ & 10.30 & $-4.53 \mathrm{E}-11$ & $8.12 \mathrm{E}-10$ \\
\hline 2.95 & $3.16 \mathrm{E}-08$ & $3.30 \mathrm{E}-08$ & 10.70 & $-4.44 \mathrm{E}-10$ & $4.03 \mathrm{E}-10$ \\
\hline 3.05 & $2.93 \mathrm{E}-08$ & $3.07 \mathrm{E}-08$ & 11.10 & $-3.56 \mathrm{E}-10$ & $2.58 \mathrm{E}-10$ \\
\hline 3.15 & $2.77 \mathrm{E}-08$ & $2.92 \mathrm{E}-08$ & 11.50 & $-1.46 \mathrm{E}-10$ & $3.20 \mathrm{E}-10$ \\
\hline 3.25 & $2.65 \mathrm{E}-08$ & $2.80 \mathrm{E}-08$ & 11.90 & $-8.02 \mathrm{E}-11$ & $4.82 E-10$ \\
\hline 3.35 & $2.59 \mathrm{E}-08$ & $2.74 E-08$ & 12.30 & $-3.92 E-11$ & $5.34 \mathrm{E}-10$ \\
\hline 3.45 & $2.59 \mathrm{E}-08$ & $2.74 \mathrm{E}-08$ & 12.70 & $-1.11 E-11$ & $4.59 \mathrm{E}-10$ \\
\hline 3.55 & $2.63 \mathrm{E}-08$ & $2.80 \mathrm{E}-08$ & 13.10 & $-3.97 \mathrm{E}-11$ & $3.79 \mathrm{E}-10$ \\
\hline 3.66 & $2.65 \mathrm{E}-08$ & $2.83 \mathrm{E}-08$ & 13.50 & $-5.03 E-11$ & $3.10 \mathrm{E}-10$ \\
\hline 3.79 & $2.53 \mathrm{E}-08$ & $2.71 \mathrm{E}-08$ & 13.90 & $-4.52 \mathrm{E}-11$ & $2.54 \mathrm{E}-10$ \\
\hline
\end{tabular}


Table A.15

Neutron Data

Detector 15 in Fig. 4

Detector Coordinates $(\mathrm{cm}): \mathrm{x}=0.0, y=0.0, \mathrm{z}^{\prime}=213.0$

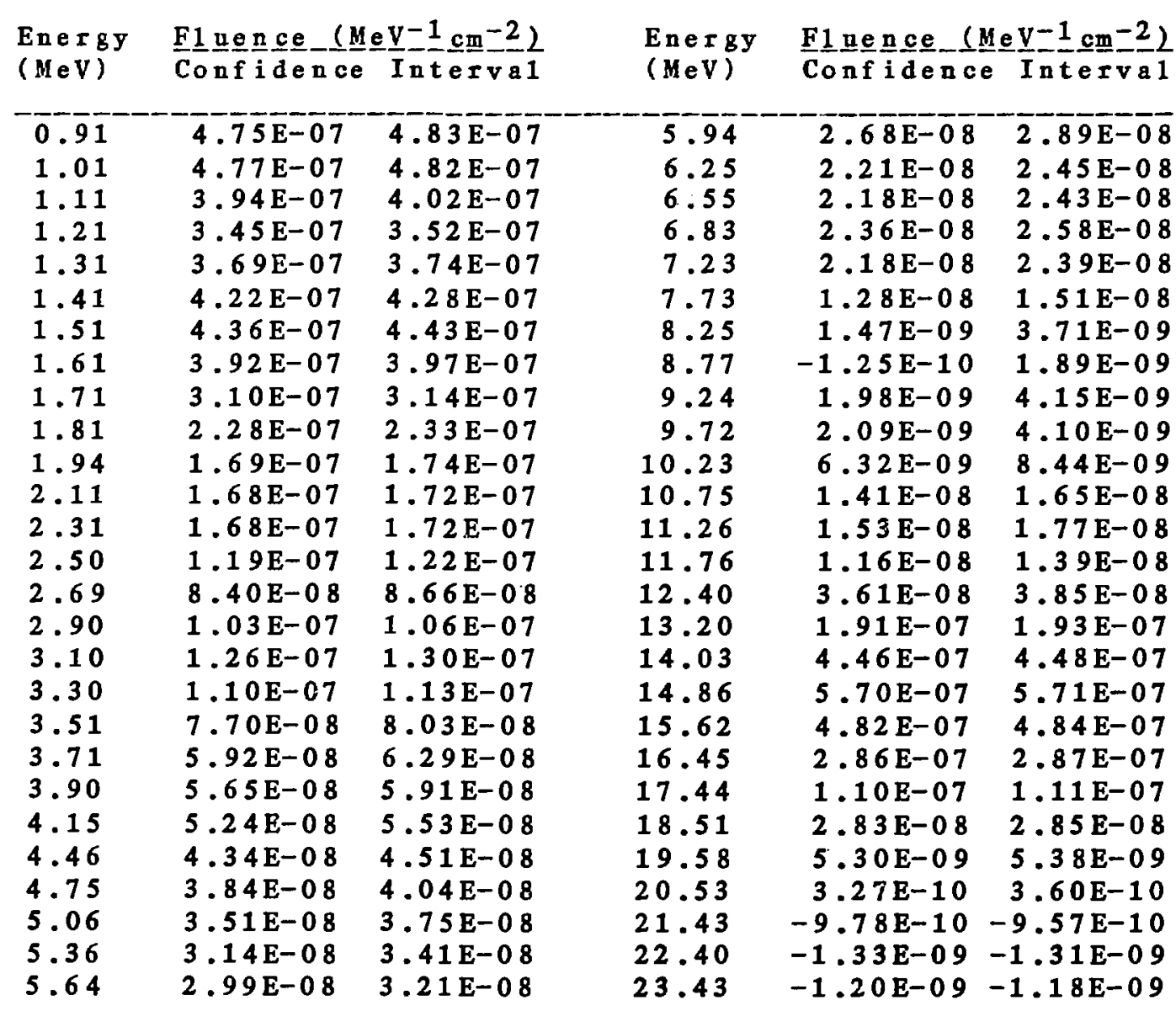


Table A.15 (Cont'd)

Gamma-Ray Data

Detector 15 in Fig. 4

Detector Coordinates $(\mathrm{cm}): \mathrm{x}=0.0, y=0.0, \mathrm{z}^{\prime}=213.0$

\begin{tabular}{|c|c|c|c|c|c|}
\hline \multirow{2}{*}{$\begin{array}{l}\text { Energy } \\
(\mathrm{MeV})\end{array}$} & \multicolumn{2}{|c|}{$\underline{F} \underline{1} \underline{\mathrm{n}} \underline{\mathrm{n}} \mathrm{c} \underline{\mathrm{e}}-\left(\underline{\mathrm{MeV}} \underline{\mathrm{v}}^{-1} \underline{\mathrm{cm}}^{-2}\right)$} & \multirow{2}{*}{$\begin{array}{l}\text { Energy } \\
(\mathrm{MeV})\end{array}$} & \multicolumn{2}{|c|}{ 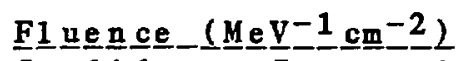 } \\
\hline & Confidence & Interva 1 & & Confidence & Interval \\
\hline 0.72 & $4.91 \mathrm{E}-07$ & $8.86 \mathrm{E}-07$ & 3.93 & $7.69 \mathrm{E}-08$ & $8.34 E-08$ \\
\hline 0.76 & $1.02 \mathrm{E}-06$ & $1.18 \mathrm{E}-06$ & 4.06 & $6.26 \mathrm{E}-08$ & $6.96 \mathrm{E}-08$ \\
\hline 0.80 & $1.36 \mathrm{E}-06$ & $1.41 \mathrm{E}-06$ & 4.19 & $5.67 \mathrm{E}-08$ & $6.41 \mathrm{E}-08$ \\
\hline 0.84 & $1.46 \mathrm{E}-06$ & $1.48 \mathrm{E}-06$ & 4.31 & $6.25 \mathrm{E}-08$ & $7.09 \mathrm{E}-08$ \\
\hline 0.88 & $1.34 \mathrm{E}-06$ & $1.36 \mathrm{E}-06$ & 4.44 & $8.08 \mathrm{E}-08$ & $9.08 \mathrm{E}-08$ \\
\hline 0.92 & $1.12 \mathrm{E}-06$ & $1.13 \mathrm{E}-06$ & 4.57 & $1.06 \mathrm{E}-07$ & $1.16 \mathrm{E}-07$ \\
\hline 0.96 & $8.99 \mathrm{E}-07$ & $9.12 \mathrm{E}-07$ & 4.70 & $1.23 \mathrm{E}-07$ & $1.34 \mathrm{E}-07$ \\
\hline 1.00 & $7.29 \mathrm{E}-07$ & $7.40 \mathrm{E}-07$ & 4.83 & $1.20 \mathrm{E}-07$ & $1.31 \mathrm{E}-07$ \\
\hline 1.04 & $6.22 \mathrm{E}-07$ & $6.32 \mathrm{E}-07$ & 4.99 & $8.92 \mathrm{E}-08$ & $1.02 \mathrm{E}-07$ \\
\hline 1.08 & $5.77 \mathrm{E}-07$ & $5.87 \mathrm{E}-07$ & 5.15 & $4.64 \mathrm{E}-08$ & $6.06 \mathrm{E}-08$ \\
\hline 1.13 & $5.93 \mathrm{E}-07$ & $6.02 \mathrm{E}-07$ & 5.32 & $2.59 \mathrm{E}-08$ & $4.10 \mathrm{E}-08$ \\
\hline 1.17 & $6.53 \mathrm{E}-07$ & $6.62 \mathrm{E}-07$ & 5.50 & $3.14 \mathrm{E}-08$ & $4.75 \mathrm{E}-08$ \\
\hline 1.22 & $6.98 \mathrm{E}-07$ & $7.08 \mathrm{E}-07$ & 5.67 & $4.86 E-08$ & $6.57 E-08$ \\
\hline 1.28 & $6.94 \mathrm{E}-07$ & $7.05 \mathrm{E}-07$ & 5.83 & $6.11 \mathrm{E}-08$ & $7.74 E-08$ \\
\hline 1.33 & $6.47 \mathrm{E}-07$ & $6.56 \mathrm{E}-07$ & 6.00 & $6.44 \mathrm{E}-08$ & $7.98 \mathrm{E}-08$ \\
\hline 1.38 & $5.75 \mathrm{E}-07$ & $5.83 \mathrm{E}-07$ & 6.18 & $6.23 \mathrm{E}-08$ & $7.72 \mathrm{E}-08$ \\
\hline 1.42 & $4.95 \mathrm{E}-07$ & $5.04 \mathrm{E}-07$ & 6.35 & $5.84 \mathrm{E}-08$ & $7.26 \mathrm{E}-08$ \\
\hline 1.47 & $4.20 \mathrm{E}-07$ & $4.27 \mathrm{E}-07$ & 6.51 & $5.54 \mathrm{E}-08$ & $6.81 \mathrm{E}-08$ \\
\hline 1.53 & $3.55 \mathrm{E}-07$ & 3. $62 \mathrm{E}-07$ & 6.70 & $5.11 \mathrm{E}-08$ & $6.18 \mathrm{E}-08$ \\
\hline 1.58 & $3.09 \mathrm{E}-07$ & $3.16 \mathrm{E}-07$ & 6.90 & $4.48 \mathrm{E}-08$ & $5.45 \mathrm{E}-08$ \\
\hline 1.64 & $2.81 \mathrm{E}-07$ & $2.89 \mathrm{E}-07$ & 7.10 & $3.93 \mathrm{E}-08$ & $4.88 \mathrm{E}-08$ \\
\hline 1.72 & $2.83 \mathrm{E}-07$ & $2.90 \mathrm{E}-07$ & 7.30 & $3.65 \mathrm{E}-08$ & $4.57 \mathrm{E}-08$ \\
\hline 1.80 & $2.93 \mathrm{E}-07$ & $3.00 E-07$ & 7.50 & $3.68 \mathrm{E}-08$ & $4.52 \mathrm{E}-08$ \\
\hline 1.88 & $2.91 \mathrm{E}-07$ & $2.98 \mathrm{E}-07$ & 7.70 & $3.70 \mathrm{E}-08$ & $4.49 \mathrm{E}-08$ \\
\hline 1.96 & $2.70 \mathrm{E}-07$ & $2.77 \mathrm{E}-07$ & 7.90 & $3.47 \mathrm{E}-08$ & $4.22 E-08$ \\
\hline 2.04 & $2.43 \mathrm{E}-07$ & $2.49 \mathrm{E}-07$ & 8.10 & $2.91 \mathrm{E}-08$ & $3.61 \mathrm{E}-08$ \\
\hline 2.12 & $2.24 \mathrm{E}-07$ & $2.31 \mathrm{E}-07$ & 8.30 & $2.17 \mathrm{E}-08$ & $2.82 \mathrm{E}-08$ \\
\hline 2.20 & $2.15 \mathrm{E}-07$ & $2.21 \mathrm{E}-07$ & 8.50 & $1.54 \mathrm{E}-08$ & $2.16 \mathrm{E}-08$ \\
\hline 2.28 & $2.02 \mathrm{E}-07$ & $2.08 \mathrm{E}-07$ & 8.73 & $1.19 \mathrm{E}-08$ & $1.75 \mathrm{E}-08$ \\
\hline 2.36 & $1.81 \mathrm{E}-07$ & $1.86 \mathrm{E}-07$ & 8.98 & $1.22 \mathrm{E}-08$ & $1.71 E-08$ \\
\hline 2.45 & $1.59 \mathrm{E}-07$ & $1.64 \mathrm{E}-07$ & 9.23 & $1.34 E-08$ & $1.80 \mathrm{E}-08$ \\
\hline 2.55 & $1.50 \mathrm{E}-07$ & $1.55 \mathrm{E}-07$ & 9.48 & $1.29 \mathrm{E}-08$ & $1.70 \mathrm{E}-08$ \\
\hline 2.65 & $1.49 \mathrm{E}-07$ & $1.54 \mathrm{E}-07$ & 9.73 & $1.01 \mathrm{E}-08$ & $1.36 \mathrm{E}-08$ \\
\hline 2.75 & $1.43 \mathrm{E}-07$ & $1.48 \mathrm{E}-07$ & 9.98 & $6.08 \mathrm{E}-09$ & $9.25 \mathrm{E}-09$ \\
\hline 2.85 & $1.32 \mathrm{E}-07$ & $1.37 \mathrm{E}-07$ & 10.30 & $1.24 \mathrm{E}-09$ & $3.89 \mathrm{E}-09$ \\
\hline 2.95 & $1.21 \mathrm{E}-07$ & $1.26 \mathrm{E}-07$ & 10.70 & $-3.40 \mathrm{E}-09$ & $-8.43 \mathrm{E}-10$ \\
\hline 3.05 & $1.15 \mathrm{E}-07$ & $1.20 \mathrm{E}-07$ & 11.10 & $-6.36 \mathrm{E}-09$ & $-3.43 \mathrm{E}-09$ \\
\hline 3.15 & $1.12 \mathrm{E}-07$ & $1.18 \mathrm{E}-07$ & 11.50 & $-7.39 \mathrm{E}-09$ & $-4.12 \mathrm{E}-09$ \\
\hline 3.25 & $1.11 \mathrm{E}-07$ & $1.17 \mathrm{E}-07$ & 11.90 & $-6.30 \mathrm{E}-09$ & $-3.21 \mathrm{E}-09$ \\
\hline 3.35 & $1.10 \mathrm{E}-07$ & $1.15 \mathrm{E}-07$ & 12.30 & $-3.59 \mathrm{E}-09$ & $-1.17 \mathrm{E}-09$ \\
\hline 3.45 & $1.09 \mathrm{E}-07$ & $1.14 \mathrm{E}-07$ & 12.70 & $-2.11 \mathrm{E}-10$ & $1.32 \mathrm{E}-09$ \\
\hline 3.55 & $1.08 \mathrm{E}-07$ & $1.14 \mathrm{E}-07$ & 13.10 & $2.67 \mathrm{E}-09$ & $3.63 \mathrm{E}-09$ \\
\hline 3.66 & $1.04 \mathrm{E}-07$ & $1.10 \mathrm{E}-07$ & 13.50 & $3.99 \mathrm{E}-09$ & $5.47 \mathrm{E}-09$ \\
\hline 3.79 & $9.31 \mathrm{E}-08$ & $9.93 \mathrm{E}-08$ & 13.90 & $4.06 \mathrm{E}-09$ & $5.85 \mathrm{E}-09$ \\
\hline
\end{tabular}


Table A.16

Neutron Data

Detector 16 in Fig. 4

Detector Coordinates $(\mathrm{cm}): \quad x=98.0, y=0.0, z^{\prime}=213.0$

\begin{tabular}{|c|c|c|c|c|c|}
\hline $\begin{array}{l}\text { Energy } \\
(\mathrm{MeV})\end{array}$ & 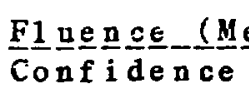 & 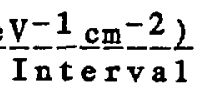 & $\begin{array}{l}\text { Energy } \\
(\mathrm{MeV})\end{array}$ & F & $\frac{v^{-1}}{I n t} \frac{c^{-2}-2}{2} \frac{2}{1}$ \\
\hline 0.91 & $1.06 \mathrm{E}-07$ & $1.07 \mathrm{E}-07$ & 5.94 & $2.75 \mathrm{E}-09$ & $2.91 \mathrm{E}-09$ \\
\hline 1.01 & $9.97 \mathrm{E}-08$ & $1.00 \mathrm{E}-07$ & 6.25 & $2.40 \mathrm{E}-09$ & $2.58 \mathrm{E}-09$ \\
\hline 1.11 & $8.94 \mathrm{E}-08$ & $9.02 \mathrm{E}-08$ & 6.55 & $2.27 \mathrm{E}-09$ & $2.46 \mathrm{E}-09$ \\
\hline 1.21 & $7.94 \mathrm{E}-08$ & $8.01 E-08$ & 6.83 & $2.21 \mathrm{E}-09$ & $2.37 E-09$ \\
\hline 1.31 & $7.07 \mathrm{E}-08$ & $7.13 \mathrm{E}-08$ & 7.23 & $2.02 \mathrm{E}-09$ & $2.17 \mathrm{E}-09$ \\
\hline 1.41 & $6.33 E-08$ & $6.39 \mathrm{E}-08$ & 7.73 & $1.66 \mathrm{E}-09$ & $1.83 \mathrm{E}-09$ \\
\hline 1.51 & $5.74 E-08$ & $5.81 E-08$ & 8.25 & $1.35 E-09$ & $1.50 E-09$ \\
\hline 1.61 & $5.16 \mathrm{E}-08$ & $5.22 \mathrm{E}-08$ & 8.77 & $1.20 \mathrm{E}-09$ & $1.33 \mathrm{E}-09$ \\
\hline 1.71 & $4.57 E-08$ & $4.62 \mathrm{E}-08$ & 9.24 & $1.12 \mathrm{E}-09$ & $1.26 \mathrm{E}-09$ \\
\hline 1.81 & $4.01 \mathrm{E}-08$ & $4.07 \mathrm{E}-08$ & 9.72 & $1.06 \mathrm{E}-09$ & $1.18 \mathrm{E}-09$ \\
\hline 1.94 & $3.48 \mathrm{E}-08$ & $3.54 \mathrm{E}-08$ & 10.23 & $1.09 \mathrm{E}-09$ & $1.22 \mathrm{E}-09$ \\
\hline 2.11 & $3.12 \mathrm{E}-08$ & $3.16 \mathrm{E}-08$ & 10.75 & $1.18 \mathrm{E}-09$ & $1.31 \mathrm{E}-09$ \\
\hline 2.31 & $2.77 \mathrm{E}-08$ & $2.81 \mathrm{E}-08$ & 11.26 & $1.13 \mathrm{E}-09$ & $1.26 \mathrm{E}-09$ \\
\hline 2.50 & $2.33 \mathrm{E}-08$ & $2.37 \mathrm{E}-08$ & 11.76 & $1.11 \mathrm{E}-09$ & $1.23 \mathrm{E}-09$ \\
\hline 2.69 & $1.91 \mathrm{E}-08$ & $1.94 \mathrm{E}-08$ & 12.40 & $1.63 \mathrm{E}-09$ & $1.75 \mathrm{E}-09$ \\
\hline 2.90 & $1.55 \mathrm{E}-08$ & $1.59 \mathrm{E}-08$ & 13.20 & $3.50 \mathrm{E}-09$ & $3.60 \mathrm{E}-09$ \\
\hline 3.10 & $1.26 \mathrm{E}-08$ & $1.30 \mathrm{E}-08$ & 14.03 & $5.55 \mathrm{E}-09$ & $5.64 E-09$ \\
\hline 3.30 & $1.02 \mathrm{E}-08$ & $1.05 \mathrm{E}-08$ & 14.86 & $5.59 \mathrm{E}-09$ & $5.67 \mathrm{E}-09$ \\
\hline 3.51 & $8.48 \mathrm{E}-09$ & $8.81 \mathrm{E}-09$ & 15.62 & $3.96 \mathrm{E}-09$ & $4.02 \mathrm{E}-09$ \\
\hline $\begin{array}{l}3.71 \\
3.90 \\
4.15\end{array}$ & $\begin{array}{l}7.48 \mathrm{E}-09 \\
6.98 \mathrm{E}-09 \\
6.17 \mathrm{E}-09\end{array}$ & $\begin{array}{l}7.84 E-09 \\
7.23 E-09 \\
6.45 E-09\end{array}$ & $\begin{array}{l}16.45 \\
17.44 \\
18.51\end{array}$ & $\begin{array}{l}1.98 \mathrm{E}-09 \\
6.55 \mathrm{E}-10 \\
1.51 \mathrm{E}-10\end{array}$ & $\begin{array}{l}2.02 \mathrm{E}-09 \\
6.75 \mathrm{E}-10 \\
1.61 \mathrm{E}-10\end{array}$ \\
\hline 4.46 & $5.06 \mathrm{E}-09$ & $5.22 \mathrm{E}-09$ & 19.58 & $2.18 \mathrm{E}-11$ & $3.09 \mathrm{E}-11$ \\
\hline 4.75 & $4.40 \mathrm{E}-09$ & $4.58 \mathrm{E}-09$ & 20.53 & $-7.28 E-12$ & $7.48 \mathrm{E}-13$ \\
\hline 5.06 & $4.05 \mathrm{E}-09$ & $4.25 \mathrm{E}-09$ & 21.43 & $-1.52 \mathrm{E}-11$ & $-8.66 E-12$ \\
\hline 5.36 & $3.59 \mathrm{E}-09$ & $3.81 \mathrm{E}-09$ & 22.40 & $-1.80 \mathrm{E}-11$ & $-1.09 \mathrm{E}-11$ \\
\hline 5.64 & $3.16 \mathrm{E}-09$ & $3.36 \mathrm{E}-09$ & 23.43 & $-1.61 \mathrm{E}-11$ & $-8.24 E-12$ \\
\hline
\end{tabular}


Table A.16 (Cont'd)

Gamma-Ray Data

Detector 16 in Fig. 4

Detector Coordinates $(\mathrm{cm}): \quad x=98.0, y=0.0, z^{\prime}=213.0$

\begin{tabular}{|c|c|c|c|c|c|}
\hline \multirow{2}{*}{$\begin{array}{l}\text { Energy } \\
(\mathrm{MeV})\end{array}$} & \multicolumn{2}{|c|}{ 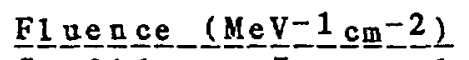 } & \multirow{2}{*}{$\begin{array}{l}\text { Energy } \\
(\mathrm{MeV})\end{array}$} & \multicolumn{2}{|c|}{ 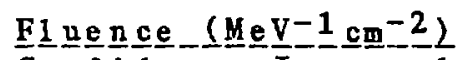 } \\
\hline & Confidence & Interva 1 & & Confidence & \\
\hline 0.72 & $8.70 \mathrm{E}-08$ & $1.53 \mathrm{E}-07$ & 3.93 & $1.72 \mathrm{E}-08$ & $1.86 \mathrm{E}-08$ \\
\hline 0.76 & $1.51 \mathrm{E}-07$ & $1.78 \mathrm{E}-07$ & 4.06 & $1.56 \mathrm{E}-08$ & $1.71 \mathrm{E}-08$ \\
\hline 0.80 & $1.84 \mathrm{E}-07$ & $1.92 \mathrm{E}-07$ & 4.19 & $1.49 \mathrm{E}-08$ & $1.65 \mathrm{E}-08$ \\
\hline 0.84 & $1.87 \mathrm{E}-07$ & $1.91 \mathrm{E}-07$ & 4.31 & $1.43 \mathrm{E}-08$ & $1.62 E-08$ \\
\hline 0.88 & $1.71 \mathrm{E}-07$ & $1.74 E-07$ & 4.44 & $1.37 \mathrm{E}-08$ & $1.59 E-08$ \\
\hline 0.92 & $1.47 \mathrm{E}-07$ & $1.50 \mathrm{E}-07$ & 4.57 & $1.37 \mathrm{E}-08$ & $1.61 \mathrm{E}-08$ \\
\hline 0.96 & $1.25 \mathrm{E}-07$ & $1.27 \mathrm{E}-07$ & 4.70 & $1.45 \mathrm{E}-08$ & $1.70 \mathrm{E}-08$ \\
\hline 1.00 & $1.08 \mathrm{E}-07$ & $1.10 \mathrm{E}-07$ & 4.83 & $1.56 \mathrm{E}-08$ & $1.82 \mathrm{E}-08$ \\
\hline 1.04 & $9.79 \mathrm{E}-08$ & $1.00 \mathrm{E}-07$ & 4.99 & $1.56 \mathrm{E}-08$ & $1.87 \mathrm{E}-08$ \\
\hline 1.08 & $9.34 \mathrm{E}-08$ & $9.53 \mathrm{E}-08$ & 5.15 & $1.31 \mathrm{E}-08$ & $1.63 \mathrm{E}-08$ \\
\hline 1.13 & $9.29 \mathrm{E}-08$ & $9.47 \mathrm{E}-08$ & 5.32 & $8.98 \mathrm{E}-09$ & $1.23 \mathrm{E}-08$ \\
\hline 1.17 & $9.47 \mathrm{E}-08$ & $9.66 \mathrm{E}-08$ & 5.50 & $6.40 \mathrm{E}-09$ & $1.01 \mathrm{E}-08$ \\
\hline 1.22 & $9.57 \mathrm{E}-08$ & $9.79 \mathrm{E}-08$ & 5.67 & $8.15 \mathrm{E}-09$ & $1.20 \mathrm{E}-08$ \\
\hline 1.28 & $9.39 \mathrm{E}-08$ & $9.62 \mathrm{E}-08$ & 5.83 & $1.38 \mathrm{E}-08$ & $1.75 E-08$ \\
\hline 1.33 & $9.03 \mathrm{E}-08$ & $9.22 \mathrm{E}-08$ & 6.00 & $2.01 \mathrm{E}-08$ & $2.34 \mathrm{E}-08$ \\
\hline 1.38 & $8.57 \mathrm{E}-08$ & $8.74 \mathrm{E}-08$ & 6.18 & $2.35 \mathrm{E}-08$ & $2.69 \mathrm{E}-08$ \\
\hline 1.42 & $8.02 \mathrm{E}-08$ & $8.20 E-08$ & 6.35 & $2.26 \mathrm{E}-08$ & $2.58 \mathrm{E}-08$ \\
\hline 1.47 & $7.39 \mathrm{E}-08$ & $7.56 \mathrm{E}-08$ & 6.51 & $1.88 \mathrm{E}-08$ & $2.15 \mathrm{E}-08$ \\
\hline 1.53 & $6.79 \mathrm{E}-08$ & $6.95 E-08$ & 6.70 & $1.42 \mathrm{E}-08$ & $1.65 \mathrm{E}-08$ \\
\hline 1.58 & $6.34 \mathrm{E}-08$ & $6.50 E-08$ & 6.90 & $1.16 \mathrm{E}-08$ & $1.37 \mathrm{E}-0.8$ \\
\hline $\begin{array}{l}1.64 \\
1.72 \\
1.80\end{array}$ & $\begin{array}{l}6.08 \mathrm{E}-08 \\
6.17 \mathrm{E}-08 \\
6.50 \mathrm{E}-08\end{array}$ & $\begin{array}{l}6.25 \mathrm{E}-08 \\
6.33 \mathrm{E}-08 \\
6.66 \mathrm{E}-08\end{array}$ & $\begin{array}{l}7.10 \\
7.30 \\
7.50\end{array}$ & $\begin{array}{l}1.20 \mathrm{E}-08 \\
1.38 \mathrm{E}-08 \\
1.50 \mathrm{E}-08\end{array}$ & $\begin{array}{l}1.40 \mathrm{E}-08 \\
1.57 \mathrm{E}-08 \\
1.67 \mathrm{E}-08\end{array}$ \\
\hline 1.88 & $6.85 \mathrm{E}-08$ & $7.01 \mathrm{E}-08$ & 7.70 & $1.41 \mathrm{E}-08$ & $1.57 \mathrm{E}-08$ \\
\hline 1.96 & $7.05 \mathrm{E}-08$ & $7.21 \mathrm{E}-08$ & & $1.13 E-08$ & $1.27 E-08$ \\
\hline 2.04 & $7.14 \mathrm{E}-08$ & $7.29 \mathrm{E}-08$ & 8.10 & $7.51 \mathrm{E}-09$ & $8.82 E-09$ \\
\hline 2.12 & $7.12 \mathrm{E}-08$ & $7.27 \mathrm{E}-08$ & 8.30 & $4.09 \mathrm{E}-09$ & $5.23 \mathrm{E}-09$ \\
\hline 2.20 & $6.86 \mathrm{E}-08$ & $7.00 \mathrm{E}-08$ & 8.50 & $1.90 \mathrm{E}-09$ & $2.90 \mathrm{E}-09$ \\
\hline 2.28 & $6.22 \mathrm{E}-08$ & $6.36 \mathrm{E}-08$ & 8.73 & $1.01 \mathrm{E}-09$ & $1.86 \mathrm{E}-09$ \\
\hline 2.36 & $5.28 \mathrm{E}-08$ & $5.41 \mathrm{E}-08$ & 8.98 & $1.17 \mathrm{E}-09$ & $1.92 \mathrm{E}-09$ \\
\hline 2.45 & $4.19 \mathrm{E}-08$ & $4.31 \mathrm{E}-08$ & 9.23 & $1.46 \mathrm{E}-09$ & $2.09 \mathrm{E}-09$ \\
\hline 2.55 & $3.34 \mathrm{E}-08$ & $3.45 \mathrm{E}-08$ & 9.48 & $1.32 \mathrm{E}-09$ & $1.89 \mathrm{E}-09$ \\
\hline 2.65 & $2.94 \mathrm{E}-08$ & $3.06 \mathrm{E}-08$ & 9.73 & $7.84 \mathrm{E}-10$ & $1.32 \mathrm{E}-09$ \\
\hline 2.75 & $2.77 \mathrm{E}-08$ & $2.89 \mathrm{E}-08$ & 9.98 & $1.37 \mathrm{E}-10$ & $6.49 \mathrm{E}-10$ \\
\hline 2.85 & $2.60 \mathrm{E}-08$ & $2.71 \mathrm{E}-08$ & $10.30^{\circ}$ & $-4.23 \mathrm{E}-10$ & $1.08 \mathrm{E}-10$ \\
\hline 2.95 & $2.38 \mathrm{E}-08$ & $2.49 \mathrm{E}-08$ & 10.70 & $-5.29 \mathrm{E}-10$ & $4.67 \mathrm{E}-11$ \\
\hline 3.05 & $2.18 \mathrm{E}-08$ & $2.30 \mathrm{E}-08$ & 11.10 & $-2.85 \mathrm{E}-10$ & $3.40 \mathrm{E}-10$ \\
\hline 3.15 & $2.07 \mathrm{E}-08$ & $2.19 \mathrm{E}-08$ & 11.50 & $-1.95 E-11$ & $4.93 \mathrm{E}-10$ \\
\hline 3.25 & $2.04 \mathrm{E}-08$ & $2.17 \mathrm{E}-08$ & 11.90 & $7.71 \mathrm{E}-11$ & $4.61 \mathrm{E}-10$ \\
\hline 3.35 & $2.05 \mathrm{E}-08$ & $2.17 \mathrm{E}-08$ & 12.30 & $3.87 \mathrm{E}-11$ & $4.39 \mathrm{E}-10$ \\
\hline 3.45 & $2.08 \mathrm{E}-08$ & $2.20 \mathrm{E}-08$ & 12.70 & $3.85 \mathrm{E}-11$ & $4.55 \mathrm{E}-10$ \\
\hline 3.55 & $2.11 \mathrm{E}-0.8$ & $2.24 E-08$ & 13.10 & $2.08 \mathrm{E}-11$ & $3.95 \mathrm{E}-10$ \\
\hline 3.66 & $2.09 \mathrm{E}-08$ & $2.23 \mathrm{E}-08$ & 13.50 & $1.67 \mathrm{E}-11$ & $3.17 \mathrm{E}-10$ \\
\hline 3.79 & $1.94 \mathrm{E}-08$ & $2.08 \mathrm{E}-08$ & 13.90 & $-4.54 E-12$ & $2.56 \mathrm{E}-10$ \\
\hline
\end{tabular}


Table A.17

Neutron Data

Detector 1 in Fig. 5 (shadow bar in place)

Detector Coordinatec $(\mathrm{cm}): x=0.0, y=0.0, z^{\prime}=151.0$

\begin{tabular}{|c|c|c|c|c|c|}
\hline $\begin{array}{l}\text { Energy } \\
(\mathrm{MeV})\end{array}$ & 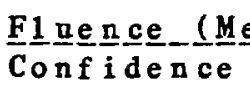 & $=\frac{V-1}{I n t e r v a l}$ & $\begin{array}{l}\text { Energy } \\
(\mathrm{MeV})\end{array}$ & 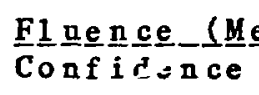 & 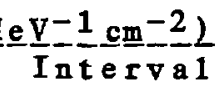 \\
\hline 0.91 & $6.02 \mathrm{E}-07$ & $6.07 \mathrm{E}-07$ & 5.94 & $2.19 \mathrm{E}-08$ & $2.28 \mathrm{E}-08$ \\
\hline 1.01 & $5.80 E-07$ & $5.83 \mathrm{E}-07$ & 6.25 & $1.97 \mathrm{E}-08$ & $2.06 \mathrm{E}-08$ \\
\hline 1.11 & $5.20 \mathrm{E}-07$ & $5.23 \mathrm{E}-07$ & 6.55 & $1.80 \mathrm{E}-08$ & $1.90 \mathrm{E}-08$ \\
\hline 1.21 & $4.60 E-07$ & $4.63 E-07$ & 6.83 & $1.68 \mathrm{E}-08$ & $1.77 \mathrm{E}-08$ \\
\hline 1.31 & $4.09 E-07$ & $4.12 \mathrm{E}-07$ & 7.23 & $1.53 \mathrm{E}-08$ & $1.61 \mathrm{E}-08$ \\
\hline 1.41 & $3.68 \mathrm{E}-07$ & $3.71 \mathrm{E}-07$ & 7.73 & $1.35 \mathrm{E}-08$ & $1.44 \mathrm{E}-08$ \\
\hline 1.51 & $3.36 \mathrm{E}-07$ & $3.39 \mathrm{E}-07$ & 8.25 & $1.04 \mathrm{E}-08$ & $1.13 \mathrm{E}-08$ \\
\hline 1.61 & $3.02 \mathrm{E}-07$ & $3.05 \mathrm{E}-07$ & 8.77 & $8.89 \mathrm{E}-09$ & $9.59 \mathrm{E}-09$ \\
\hline 1.71 & $2.68 \mathrm{E}-07$ & $2.70 \mathrm{E}-07$ & 9.24 & $8.75 \mathrm{E}-09$ & $9.50 \mathrm{E}-09$ \\
\hline 1.81 & $2.36 \mathrm{E}-07$ & $2.38 \mathrm{E}-07$ & 9.72 & $8.56 \mathrm{E}-09$ & $9.23 \mathrm{E}-09$ \\
\hline 1.94 & $2.05 \mathrm{E}-07$ & $2.08 \mathrm{E}-07$ & 10.23 & $8.40 \mathrm{E}-09$ & $9.10 \mathrm{E}-09$ \\
\hline 2.11 & $1.81 \mathrm{E}-07$ & $1.83 \mathrm{E}-07$ & 10.75 & $8.53 E-09$ & $9.28 \mathrm{E}-09$ \\
\hline 2.31 & $1.56 \mathrm{E}-07$ & $1.58 \mathrm{E}-07$ & 11.26 & $8.87 \mathrm{E}-09$ & $9.62 \mathrm{E}-09$ \\
\hline 2.50 & $1.30 \mathrm{E}-07$ & $1.32 \mathrm{E}-07$ & 11.76 & $1.05 \mathrm{E}-08$ & $1.13 E-08$ \\
\hline 2.69 & $1.11 \mathrm{E}-07$ & $1.13 \mathrm{E}-07$ & 12.40 & $1.81 \mathrm{E}-0.8$ & $1.88 \mathrm{E}-08$ \\
\hline 2.90 & $1.00 \mathrm{E}-07$ & $1.01 \mathrm{E}-07$ & 13.20 & $4.03 \mathrm{E}-08$ & $4.10 \mathrm{E}-08$ \\
\hline 3.10 & $8.78 \mathrm{E}-08$ & $8.97 \mathrm{E}-08$ & 14.03 & $6.70 \mathrm{E}-08$ & $6.75 \mathrm{E}-08$ \\
\hline 3.30 & $7.44 \mathrm{E}-08$ & $7.57 \mathrm{E}-08$ & 14.86 & $7.03 \mathrm{E}-08$ & $7.07 \mathrm{E}-08$ \\
\hline 3.51 & $6.22 \mathrm{E}-08$ & $6.38 \mathrm{E}-08$ & 15.62 & $5.10 \mathrm{E}-08$ & $5.14 \mathrm{E}-08$ \\
\hline 3.71 & $5.48 \mathrm{E}-08$ & $5.66 \mathrm{E}-08$ & 16.45 & $2.59 \mathrm{E}-08$ & $2.61 E-08$ \\
\hline $\begin{array}{l}3.90 \\
4.15\end{array}$ & $\begin{array}{l}5.12 \mathrm{E}-08 \\
4.61 \mathrm{E}-08\end{array}$ & $\begin{array}{l}5.24 \mathrm{E}-08 \\
4.75 \mathrm{E}-08\end{array}$ & $\begin{array}{l}17.44 \\
18.51\end{array}$ & $\begin{array}{l}8.48 \mathrm{E}-09 \\
1.88 \mathrm{E}-09\end{array}$ & $\begin{array}{l}8.60 \mathrm{E}-09 \\
1.94 \mathrm{E}-09\end{array}$ \\
\hline 4.46 & $3.89 \mathrm{E}-08$ & $3.96 \mathrm{E}-08$ & 19.58 & $2.73 \mathrm{E}-10$ & $3.22 \mathrm{E}-10$ \\
\hline 4.75 & $3.29 \mathrm{E}-08$ & $3.38 \mathrm{E}-08$ & 20.53 & $-6.55 \mathrm{E}-11$ & $-2.26 \mathrm{E}-11$ \\
\hline 5.06 & $2.85 \mathrm{E}-08$ & $2.95 \mathrm{E}-08$ & 21.43 & $-1.61 \mathrm{E}-10$ & $-1.26 \mathrm{E}-10$ \\
\hline 5.36 & $2.53 \mathrm{E}-08$ & $2.65 \mathrm{E}-08$ & 22.40 & $-1.93 E-10$ & $-1.55 \mathrm{E}-10$ \\
\hline 5.64 & $2.37 \mathrm{E}-08$ & $2.46 \mathrm{E}-08$ & 23.43 & $-1.73 \mathrm{E}-10$ & $-1.30 \mathrm{E}-10$ \\
\hline
\end{tabular}


Table A.17 (Cont'd)

Gamma-Ray Data

Detector 1 in Fig. 5 (shadow bar in place)

Detector Coordinates $(\mathrm{cm}): x=0.0, y=0.0, z^{\prime}=151.0$

\begin{tabular}{|c|c|c|c|c|c|}
\hline \multirow{2}{*}{$\begin{array}{l}\text { Energy } \\
(\mathrm{MeV})\end{array}$} & \multicolumn{2}{|c|}{ 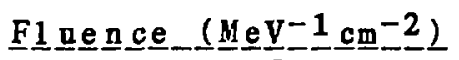 } & \multirow{2}{*}{$\begin{array}{l}\text { Energy } \\
(\mathrm{MeV})\end{array}$} & \multicolumn{2}{|c|}{ 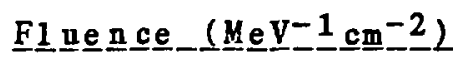 } \\
\hline & Confidence & Interva 1 & & Confidence & Interva 1 \\
\hline 0.72 & $5.31 \mathrm{E}-07$ & $8.29 \mathrm{E}-07$ & 3.93 & $6.39 \mathrm{E}-08$ & $6 . \overline{82}-08$ \\
\hline 0.76 & $8.59 \mathrm{E}-07$ & $9.79 \mathrm{E}-07$ & 4.06 & $5.58 \mathrm{E}-08$ & $6.04 \mathrm{E}-08$ \\
\hline 0.80 & $1.03 \mathrm{E}-06$ & $1.07 \mathrm{E}-06$ & 4.19 & $5.17 \mathrm{E}-08$ & $5.66 \mathrm{E}-08$ \\
\hline 0.84 & $1.04 E-06$ & $1.06 \mathrm{E}-06$ & 4.31 & $5.12 \mathrm{E}-08$ & $5.69 \mathrm{E}-08$ \\
\hline 0.88 & $9.40 \mathrm{E}-07$ & $9.50 \mathrm{E}-07$ & 4.44 & $5.38 \mathrm{E}-08$ & $6.05 \mathrm{E}-08$ \\
\hline 0.92 & $7.94 \mathrm{E}-07$ & $8.04 \mathrm{E}-07$ & 4.57 & $5.80 \mathrm{E}-08$ & $6.52 \mathrm{E}-08$ \\
\hline 0.96 & $6.59 \mathrm{E}-07$ & $6.68 \mathrm{E}-07$ & 4.70 & $6.08 \mathrm{E}-08$ & $6.80 \mathrm{E}-08$ \\
\hline 1.00 & $5.60 \mathrm{E}-07$ & $5.67 \mathrm{E}-07$ & 4.83 & $5.88 \mathrm{E}-08$ & $6.64 \mathrm{E}-08$ \\
\hline 1.04 & $4.99 \mathrm{E}-07$ & $5.06 \mathrm{E}-07$ & 4.99 & $4.95 \mathrm{E}-08$ & $5.87 \mathrm{E}-08$ \\
\hline 1.08 & $4.71 \mathrm{E}-07$ & $4.77 \mathrm{E}-07$ & 5.15 & $3.56 \mathrm{E}-08$ & $4.54 \mathrm{E}-08$ \\
\hline 1.13 & $4.66 \mathrm{E}-07$ & $4.73 \mathrm{E}-07$ & 5.32 & $2.66 \mathrm{E}-08$ & $3.64 \mathrm{E}-08$ \\
\hline 1.17 & $4.76 \mathrm{E}-07$ & $4.83 \mathrm{E}-07$ & 5.50 & $2.74 \mathrm{E}-08$ & $3.83 \mathrm{E}-08$ \\
\hline 1.22 & $4.78 \mathrm{E}-07$ & $4.85 \mathrm{E}-07$ & 5.67 & $3.84 \mathrm{E}-08$ & $4.99 \mathrm{E}-08$ \\
\hline 1.28 & $4.57 \mathrm{E}-07$ & $4.65 \mathrm{E}-07$ & 5.83 & $5.25 \mathrm{E}-08$ & $6.33 \mathrm{E}-08$ \\
\hline 1.33 & $4.18 \mathrm{E}-07$ & $4.25 \mathrm{E}-07$ & 6.00 & $6.14 \mathrm{E}-08$ & $7.14 \mathrm{E}-08$ \\
\hline 1.38 & $3.72 \mathrm{E}-07$ & $3.77 \mathrm{E}-07$ & 6.18 & $6.14 \mathrm{E}-08$ & $7.14 E-08$ \\
\hline 1.42 & $3.28 \mathrm{E}-07$ & $3.34 \mathrm{E}-07$ & 6.35 & $5.44 \mathrm{E}-08$ & $6.36 \mathrm{E}-08$ \\
\hline 1.47 & $2.92 \mathrm{E}-07$ & $2.98 \mathrm{E}-07$ & 6.51 & $4.54 \mathrm{E}-08$ & $5.37 \mathrm{E}-08$ \\
\hline 1.53 & $2.66 \mathrm{E}-07$ & $2.71 \mathrm{E}-07$ & 6.70 & $3.90 \mathrm{E}-08$ & $4.61 \mathrm{E}-08$ \\
\hline 1.58 & $2.50 \mathrm{E}-07$ & $2.56 \mathrm{E}-07$ & 6.90 & $3.76 \mathrm{E}-08$ & $4.39 E-08$ \\
\hline 1.64 & $2.44 \mathrm{E}-07$ & $2.49 \mathrm{E}-07$ & 7.10 & $3.96 \mathrm{E}-08$ & $4.56 \mathrm{E}-08$ \\
\hline $\begin{array}{l}1.72 \\
1.80\end{array}$ & $\begin{array}{l}2.48 \mathrm{E}-07 \\
2.55 \mathrm{E}-07\end{array}$ & $\begin{array}{l}2.53 \mathrm{E}-07 \\
2.60 \mathrm{E}-07\end{array}$ & $\begin{array}{l}7.30 \\
7.50\end{array}$ & $\begin{array}{l}4.11 \mathrm{E}-08 \\
3.94 \mathrm{E}-08\end{array}$ & $\begin{array}{l}4.66 \mathrm{E}-08 \\
4.45 \mathrm{E}-08\end{array}$ \\
\hline 1.88 & $2.57 \mathrm{E}-07$ & $2.62 \mathrm{E}-07$ & 7.70 & $3.39 \mathrm{E}-08$ & $3.88 \mathrm{E}-08$ \\
\hline 1.96 & $2.49 \mathrm{E}-07$ & $2.55 \mathrm{E}-07$ & 7.90 & $2.63 \mathrm{E}-08$ & $3.07 \mathrm{E}-08$ \\
\hline 2.04 & $2.38 \mathrm{E}-07$ & $2.42 \mathrm{E}-07$ & & $1.87 \mathrm{E}-08$ & $2.26 \mathrm{E}-08$ \\
\hline 2.12 & $2.29 \mathrm{E}-07$ & $2.33 \mathrm{E}-07$ & 8.30 & $1.26 \mathrm{E}-08$ & $1.60 \mathrm{E}-08$ \\
\hline 2.20 & $2.19 \mathrm{E}-07$ & $2.23 \mathrm{E}-07$ & 8.50 & $8.81 \mathrm{E}-09$ & $1.18 \mathrm{E}-08$ \\
\hline 2.28 & $2.01 \mathrm{E}-07$ & $2.05 \mathrm{E}-07$ & 8.73 & $6.56 \mathrm{E}-09$ & $9.28 \mathrm{E}-09$ \\
\hline 2.36 & $1.76 \mathrm{E}-07$ & $1.80 \mathrm{E}-07$ & 8.98 & $5.64 \mathrm{E}-09$ & $8.09 \mathrm{E}-09$ \\
\hline 2.45 & $1.49 \mathrm{E}-07$ & $1.53 \mathrm{E}-07$ & 9.23 & $5.04 \mathrm{E}-09$ & $7.11 \mathrm{E}-09$ \\
\hline 2.55 & $1.32 E-07$ & $1.36 \mathrm{E}-07$ & 9.48 & $4.07 \mathrm{E}-09$ & $5.95 \mathrm{E}-09$ \\
\hline 2.65 & $1.23 \mathrm{E}-07$ & $1.26 \mathrm{E}-07$ & 9.73 & $2.86 \mathrm{E}-09$ & $4.54 \mathrm{E}-09$ \\
\hline 2.75 & $1.14 \mathrm{E}-07$ & $1.18 \mathrm{E}-07$ & 9.98 & $1.51 \mathrm{E}-09$ & $3.28 \mathrm{E}-09$ \\
\hline 2.85 & $1.05 \mathrm{E}-07$ & $1.09 \mathrm{E}-07$ & 10.30 & $1.30 \mathrm{E}-10$ & $2.09 \mathrm{E}-09$ \\
\hline 2.95 & $9.70 \mathrm{E}-08$ & $1.01 \mathrm{E}-07$ & 10.70 & $-6.53 \mathrm{E}-10$ & $1.46 \mathrm{E}-09$ \\
\hline 3.05 & $9.08 \mathrm{E}-08$ & $9.44 \mathrm{E}-08$ & 11.10 & $-5.97 \mathrm{E}-10$ & $1.26 \mathrm{E}-09$ \\
\hline 3.15 & & $9.11 \mathrm{E}-08$ & 11.50 & $-2.67 \mathrm{E}-10$ & $1.03 \mathrm{E}-09$ \\
\hline 3.25 & $8.63 \mathrm{E}-08$ & $9.01 \mathrm{E}-08$ & 11.90 & $-1.19 \mathrm{E}-10$ & $1.01 \mathrm{E}-09$ \\
\hline 3.35 & $8.63 \mathrm{E}-08$ & $9.01 \mathrm{E}-08$ & 12.30 & $-1.41 \mathrm{E}-10$ & $1.21 \mathrm{E}-09$ \\
\hline 3.45 & $8.61 \mathrm{E}-08$ & $8.99 \mathrm{E}-08$ & 12.70 & $-4.25 \mathrm{E}-11$ & $1.20 \mathrm{E}-09$ \\
\hline 3.55 & $8.49 \mathrm{E}-08$ & $8.89 E-08$ & 13.10 & $-8.95 \mathrm{E}-11$ & $1.01 \mathrm{E}-09$ \\
\hline 3.66 & $8.12 \mathrm{E}-08$ & $8.55 \mathrm{E}-0.8$ & 13.50 & $-1.04 \mathrm{E}-10$ & $7.97 \mathrm{E}-10$ \\
\hline 3.79 & $7.35 \mathrm{E}-08$ & $7.78 E-08$ & 13.90 & $-1.01 \mathrm{E}-10$ & $6.64 \mathrm{E}-10$ \\
\hline
\end{tabular}


Table A. 18

Neutron Data

Detector 2 in Fig. 5 (shadow bar in place)

Detector Coordinates $(\mathrm{cm}): \quad x=0.0, y=0.0, z^{\prime}=181.0$

\begin{tabular}{|c|c|c|c|c|c|}
\hline \multirow{2}{*}{$\begin{array}{l}\text { Energy } \\
(\mathrm{MeV})\end{array}$} & \multicolumn{2}{|c|}{ F1 uence $\left(M e V-1 c m^{-2}\right)$} & \multirow{2}{*}{$\begin{array}{l}\text { Energy } \\
\text { (MeV) }\end{array}$} & \multicolumn{2}{|c|}{ F1 uㅡ를 $\left(\underline{M e V}-1 \mathrm{~cm}^{-2}\right)$} \\
\hline & Confidence & Interval & & Confidence & Interval \\
\hline 0.91 & $3.76 \mathrm{E}-07$ & $3.79 E-07$ & 5.94 & $1.43 \mathrm{E}-$ & $1.48 \mathrm{E}-08$ \\
\hline 1.01 & $3.65 \mathrm{E}-07$ & $3.67 \mathrm{E}-07$ & 6.25 & $1.25 \mathrm{E}-08$ & $1.30 \mathrm{E}-08$ \\
\hline 1.11 & $3.31 \mathrm{E}-07$ & $3.33 \mathrm{E}-07$ & 6.55 & $1.16 \mathrm{E}-08$ & $1.22 \mathrm{E}-08$ \\
\hline 1.21 & $2.95 \mathrm{E}-07$ & $2.97 \mathrm{E}-07$ & 6.83 & $1.11 \mathrm{E}-08$ & $1.16 \mathrm{E}-08$ \\
\hline 1.31 & $2.63 \mathrm{E}-07$ & $2.65 \mathrm{E}-07$ & 7.23 & $1.02 E-08$ & $1.06 \mathrm{E}-08$ \\
\hline 1.41 & $2.38 \mathrm{E}-07$ & $2.40 \mathrm{E}-07$ & 7.73 & $8.03 \mathrm{E}-09$ & $8.59 \mathrm{E}-09$ \\
\hline 1.51 & $2.17 \mathrm{E}-07$ & $2.19 \mathrm{E}-07$ & 8.25 & $5.99 \mathrm{E}-09$ & $6.49 \mathrm{E}-09$ \\
\hline 1.61 & $1.96 \mathrm{E}-07$ & $1.98 \mathrm{E}-07$ & 8.77 & $5.73 \mathrm{E}-09$ & $6.15 \mathrm{E}-09$ \\
\hline 1.71 & $1.74 E-07$ & $1.75 \mathrm{E}-07$ & 9.24 & $5.91 E-09$ & $6.36 \mathrm{E}-09$ \\
\hline 1.81 & $1.54 \mathrm{E}-07$ & $1.56 \mathrm{E}-07$ & 9.72 & $5.75 \mathrm{E}-09$ & $6.15 \mathrm{E}-09$ \\
\hline 1.94 & $1.35 \mathrm{E}$ & $1.37 \mathrm{E}-07$ & 10.23 & $5.68 \mathrm{E}$ & 6.1 \\
\hline 2.1 & 1.2 & 1. $22 \mathrm{E}-07$ & 10.75 & 5.8 & -09 \\
\hline 2.31 & $1.05 \mathrm{E}-07$ & $1.06 \mathrm{E}-07$ & 11.26 & $6.04 \mathrm{E}-09$ & $6.49 \mathrm{E}-09$ \\
\hline 2.50 & $8.74 \mathrm{E}-08$ & $8.84 \mathrm{E}-08$ & 11.76 & $6.77 \mathrm{E}-09$ & $7.21 \mathrm{E}-09$ \\
\hline 2.69 & $7.43 \mathrm{E}-08$ & $7.51 \mathrm{E}-08$ & 12.40 & $1.11 \mathrm{E}-08$ & $1.15 \mathrm{E}-08$ \\
\hline 2.90 & $6.55 \mathrm{E}-08$ & $6.64 \mathrm{E}-08$ & 13.20 & $2.50 \mathrm{E}-08$ & $2.53 \mathrm{E}-08$ \\
\hline 3.10 & $5.69 \mathrm{E}-08$ & $5.81 \mathrm{E}-0.8$ & 14.03 & $4.17 \mathrm{E}-08$ & $4.21 E-08$ \\
\hline 3.30 & $4.88 E-08$ & $4.96 \mathrm{E}-08$ & 14.86 & $4.37 E-08$ & $4.40 \mathrm{E}-08$ \\
\hline 3.51 & $4.22 \mathrm{E}-08$ & $4.32 \mathrm{E}-08$ & 15.62 & $3.16 E-08$ & $3.18 \mathrm{E}-08$ \\
\hline 3.71 & $3.75 E-08$ & $3.86 \mathrm{E}-08$ & 16.45 & $1.60 \mathrm{E}-08$ & $1.62 \mathrm{E}-08$ \\
\hline 3.90 & $3.40 E-08$ & $3.48 \mathrm{E}-08$ & 17.44 & $5.27 \mathrm{E}-09$ & $5.34 E-09$ \\
\hline 4.15 & $2.93 \mathrm{E}-08$ & $3.02 \mathrm{E}-08$ & 18.51 & $1.18 \mathrm{E}-09$ & $1.21 \mathrm{E}-09$ \\
\hline 4.46 & $2.47 \mathrm{E}-08$ & $2.52 \mathrm{E}-0.8$ & 19.58 & $1.74 \mathrm{E}-10$ & $2.04 \mathrm{E}-10$ \\
\hline 4.75 & $2.16 \mathrm{E}-08$ & $2.21 \mathrm{E}-08$ & 20.53 & $-4.03 E-11$ & $-1.39 E-11$ \\
\hline 5.06 & $1.91 \mathrm{E}-08$ & $1.97 \mathrm{E}-08$ & 21.43 & $-1.01 \mathrm{E}-10$ & $-7.96 E-11$ \\
\hline 5.36 & $1.71 \mathrm{E}-08$ & $1.78 \mathrm{E}-08$ & 22.40 & $-1.21 \mathrm{E}-10$ & $-9.73 E-11$ \\
\hline 5.64 & $1.58 \mathrm{E}-08$ & $1.64 \mathrm{E}-08$ & 23.43 & $-1.08 \mathrm{E}-10$ & $-8.17 E-11$ \\
\hline
\end{tabular}


Table A.18 (Cont'd)

Gamma-Ray Data

Detector 2 in Fig. 5 (shadow bar in place)

Detector Coordinates $(\mathrm{cm}): \mathrm{x}=0.0, y=0.0, \mathrm{z}^{\prime}=181.0$

\begin{tabular}{|c|c|c|c|c|c|}
\hline \multirow{2}{*}{$\begin{array}{l}\text { Energy } \\
(\mathrm{MeV})\end{array}$} & \multicolumn{2}{|c|}{ 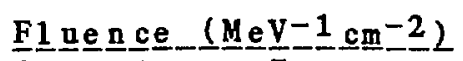 } & \multirow{2}{*}{$\begin{array}{l}\text { Energy } \\
(\mathrm{MeV})\end{array}$} & \multicolumn{2}{|c|}{$\underline{F} \underline{1} \underline{\underline{u}} \underline{\mathrm{e}} \underline{\underline{n}} \underline{\mathrm{c}} \underline{\mathrm{e}}-\left(\underline{\mathrm{Me}} \underline{\mathrm{V}}^{-1} \mathrm{c} \underline{\underline{\mathrm{m}}-2}\right)$} \\
\hline & Conf idence & Interva 1 & & Confidence & InterTa 1 \\
\hline 0.72 & $3.65 \mathrm{E}-07$ & $5.67 \mathrm{E}-07$ & 3.93 & $4.56 \mathrm{E}-08$ & $4.84 E-08$ \\
\hline 0.76 & $5.91 \mathrm{E}-07$ & $6.73 \mathrm{E}-07$ & 4.06 & $4.03 \mathrm{E}-08$ & $4.33 E-08$ \\
\hline 0.80 & $7.18 \mathrm{E}-07$ & $7.42 \mathrm{E}-07$ & 4.19 & $3.80 \mathrm{E}-08$ & $4.12 E-08$ \\
\hline 0.84 & $7.30 \mathrm{E}-07$ & $7.38 \mathrm{E}-07$ & 4.31 & $3.78 \mathrm{E}-08$ & $4.15 E-08$ \\
\hline 0.88 & $6.55 \mathrm{E}-07$ & $6.61 \mathrm{E}-07$ & 4.44 & $3.83 \mathrm{E}-08$ & $4.26 E-08$ \\
\hline 0.92 & $5.46 \bar{E}-07$ & $5.52 \mathrm{E}-07$ & 4.57 & $3.87 \mathrm{E}-08$ & $4.33 E-08$ \\
\hline 0.96 & $4.46 \mathrm{E}-07$ & $4.51 \mathrm{E}-07$ & 4.70 & $3.90 \mathrm{E}-08$ & $4.37 E-0 \mathrm{~s}$ \\
\hline 1.00 & $3.76 \mathrm{E}-07$ & $3.80 \mathrm{E}-07$ & 4.83 & $3.86 \mathrm{E}-08$ & $4.35 E-08$ \\
\hline 1.04 & $3.35 \mathrm{E}-07$ & $3.39 \mathrm{E}-07$ & 4.99 & $3.61 \mathrm{E}-08$ & $4.19 E-08$ \\
\hline 1.08 & $3.18 \mathrm{E}-07$ & $3.22 \mathrm{E}-07$ & 5.15 & $3.00 \mathrm{E}-08$ & $3.62 \mathrm{E}-08$ \\
\hline 1.13 & $3.19 \mathrm{E}-07$ & $3.22 \mathrm{E}-07$ & 5.32 & $2.32 \mathrm{E}-08$ & $2.95 \mathrm{E}-08$ \\
\hline 1.17 & $3.29 \mathrm{E}-07$ & $3.33 \mathrm{E}-07$ & 5.50 & $2.01 \mathrm{E}-08$ & $2.71 E-08$ \\
\hline 1.22 & $3.32 \mathrm{E}-07$ & $3.36 \mathrm{E}-07$ & 5.67 & $2.43 \mathrm{E}-08$ & $3.17 E-08$ \\
\hline 1.28 & $3.17 \mathrm{E}-07$ & $3.22 \mathrm{E}-07$ & 5.83 & $3.33 \mathrm{E}-08$ & $4.03 E-08$ \\
\hline 1.33 & $2.87 \mathrm{E}-07$ & $2.91 \mathrm{E}-07$ & 6.00 & $4.17 \mathrm{E}-08$ & $4.81 E-08$ \\
\hline 1.38 & $2.50 \mathrm{E}-07$ & $2.54 \mathrm{E}-07$ & 6.18 & $4.50 \mathrm{E}-08$ & $5.14 \mathrm{E}-08$ \\
\hline 1.42 & $2.17 \mathrm{E}-07$ & $2.21 \mathrm{E}-07$ & 6.35 & $4.28 \mathrm{E}-08$ & $4.87 E-08$ \\
\hline 1.47 & $1.91 \mathrm{E}-07$ & $1.95 \mathrm{E}-07$ & 6.51 & 3.7 & $4 E-08$ \\
\hline 1.53 & 1.7 & $1.77 \mathrm{E}-07$ & & & $3.49 \mathrm{E}-08$ \\
\hline 1.58 & $1.65 \mathrm{E}-07$ & $1.68 \mathrm{E}-07$ & 6.90 & $2.57 \mathrm{E}-08$ & $2.97 \mathrm{E}-08$ \\
\hline 1.64 & $1.64 \mathrm{E}-07$ & $1.67 \mathrm{E}-07$ & 7.10 & $2.47 \mathrm{E}-08$ & $2.85 \mathrm{E}-08$ \\
\hline 1.72 & $1.70 \mathrm{E}-07$ & $1.73 \mathrm{E}-07$ & 7.30 & $2.57 \mathrm{E}-08$ & $2.92 \mathrm{E}-08$ \\
\hline 1.80 & $1.74 \mathrm{E}-07$ & $1.77 \mathrm{E}-07$ & 7.50 & $2.62 \mathrm{E}-08$ & $2.94 \mathrm{E}-08$ \\
\hline 1.88 & $1.73 \mathrm{E}-07$ & $1.76 \mathrm{E}-07$ & 7.70 & $2.39 E-08$ & $2.71 \mathrm{E}-08$ \\
\hline 1.96 & $1.70 \mathrm{E}-07$ & $1.73 \mathrm{E}-07$ & 7.90 & $1.96 \mathrm{E}-08$ & $2.23 \mathrm{E}-08$ \\
\hline 2.04 & $1.65 \mathrm{E}-07$ & $1.69 \mathrm{E}-07$ & 8.10 & $1.41 \mathrm{E}-08$ & $1.66 \mathrm{E}-08$ \\
\hline 2.12 & $1.59 \mathrm{E}-07$ & $1.62 \mathrm{E}-07$ & 8.30 & $9.18 \mathrm{E}-$ & $1.14 \mathrm{E}-08$ \\
\hline 2.20 & $1.49 \mathrm{E}-07$ & $1.52 \mathrm{E}-07$ & 8.50 & $5.88 \mathrm{E}-09$ & $7.82 \mathrm{E}-09$ \\
\hline 2.28 & $1.35 \mathrm{E}-07$ & $1.38 \mathrm{E}-07$ & 8.73 & $4.09 \mathrm{E}-09$ & $5.88 \mathrm{E}-09$ \\
\hline 2.36 & $1.18 \mathrm{E}-07$ & $1.21 \mathrm{E}-07$ & 8.98 & $3.90 \mathrm{E}-09$ & $5.48 \mathrm{E}-09$ \\
\hline 2.45 & $1.02 \mathrm{E}-07$ & $1.05 \mathrm{E}-07$ & 9.23 & $4.07 \mathrm{E}-09$ & $5.43 \mathrm{E}-09$ \\
\hline 2.55 & $9.07 \mathrm{E}-08$ & $9.30 \mathrm{E}-08$ & 9.48 & $3.62 \mathrm{E}-09$ & $4.84 \mathrm{E}-09$ \\
\hline 2.65 & $8.34 \mathrm{E}-08$ & $8.58 \mathrm{E}-08$ & 9.73 & $2.56 \mathrm{E}-09$ & $3.66 \mathrm{E}-09$ \\
\hline 2.75 & $7.72 \mathrm{E}-08$ & $7.95 \mathrm{E}-08$ & 9.98 & 1.24 E- 09 & $2.38 \mathrm{E}-09$ \\
\hline & $7.15 \mathrm{E}-08$ & & 10.30 & $-4.37 \mathrm{E}-11$ & $1.21 \mathrm{E}-09$ \\
\hline $\begin{array}{l}2.95 \\
3.05\end{array}$ & $\begin{array}{l}6.69 E-08 \\
6.31 E-08\end{array}$ & $\begin{array}{l}6.91 \mathrm{E}-08 \\
6.53 \mathrm{E}-08\end{array}$ & $\begin{array}{l}10.70 \\
11.10\end{array}$ & $\begin{array}{l}-5.91 E-10 \\
-4.14 E-10\end{array}$ & $\begin{array}{l}7.54 \mathrm{E}-10 \\
7.73 \mathrm{E}-10\end{array}$ \\
\hline $\begin{array}{l}3.15 \\
3.25\end{array}$ & $\begin{array}{l}6.00 \mathrm{E}-08 \\
5.84 \mathrm{E}-08\end{array}$ & $\begin{array}{l}6.24 \mathrm{E}-08 \\
6.08 \mathrm{E}-08\end{array}$ & $\begin{array}{l}11.50 \\
11.90\end{array}$ & $\begin{array}{l}-1.23 \mathrm{E}-10 \\
-1.77 \mathrm{E}-11\end{array}$ & $\begin{array}{l}7.03 \mathrm{E}-10 \\
6.94 \mathrm{E}-10\end{array}$ \\
\hline 3.35 & $5.86 E-08$ & $6.10 \mathrm{E}-08$ & 12.30 & $-3.72 E-11$ & $\begin{array}{l}0.94 \mathrm{E}-10 \\
\text { B. } 17 \mathrm{E}-10\end{array}$ \\
\hline & $5.97 E-08$ & $6.22 E-08$ & 12.70 & $1.84 \mathrm{E}-11$ & 8. $02 \mathrm{E}-10$ \\
\hline 3.55 & $6.02 \mathrm{E}-08$ & $6.28 \mathrm{E}-08$ & 13.10 & $-2.47 \mathrm{E}-11$ & $6.74 \mathrm{E}-10$ \\
\hline 3.66 & $5.82 \mathrm{E}-08$ & $6.10 \mathrm{E}-08$ & 13.50 & $-4.79 E-11$ & $5.25 \mathrm{E}-10$ \\
\hline 3.79 & $5.26 \mathrm{E}-08$ & $5.53 \mathrm{E}-08$ & 13.90 & $-5.54 \mathrm{E}-11$ & $4.30 \mathrm{E}-10$ \\
\hline
\end{tabular}


Table A.19

Neutron Data

Detector 3 in Fig. 5 (shadow bax in place)

Detector Coordinates $(\mathrm{cm}): \mathrm{x}=0.0, \mathrm{y}=0.0, \mathrm{z}^{\prime}=213.0$

\begin{tabular}{|c|c|c|c|c|c|}
\hline $\begin{array}{l}\text { Energy } \\
(\mathrm{MeV})\end{array}$ & 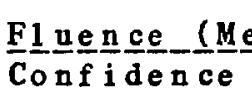 & 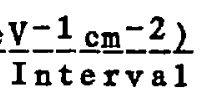 & $\begin{array}{l}\text { Energy } \\
(\mathrm{MeV})\end{array}$ & $\frac{\text { Fl }}{\text { Conf }} \underline{\text { n }} \frac{\text { nce }}{\text { idence }}$ & 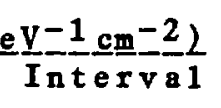 \\
\hline $\begin{array}{l}0.91 \\
1.01 \\
1.11 \\
1.21 \\
1.31 \\
1.41 \\
1.51 \\
1.61 \\
1.71 \\
1.81 \\
1.94 \\
2.11 \\
2.31 \\
2.50 \\
2.69 \\
2.90 \\
3.10 \\
3.30 \\
3.51 \\
3.71 \\
3.90 \\
4.15 \\
4.46 \\
4.75 \\
5.06 \\
5.36 \\
5.64\end{array}$ & $\begin{array}{l}2.59 \mathrm{E}-07 \\
2.51 \mathrm{E}-07 \\
2.28 \mathrm{E}-07 \\
2.04 \mathrm{E}-07 \\
1.83 \mathrm{E}-07 \\
1.65 \mathrm{E}-07 \\
1.51 \mathrm{E}-07 \\
1.36 \mathrm{E}-07 \\
1.22 \mathrm{E}-07 \\
1.08 \mathrm{E}-07 \\
9.43 \mathrm{E}-08 \\
8.43 \mathrm{E}-08 \\
7.31 \mathrm{E}-08 \\
6.11 \mathrm{E}-08 \\
5.22 \mathrm{E}-08 \\
4.63 \mathrm{E}-08 \\
4.01 \mathrm{E}-08 \\
3.35 \mathrm{E}-08 \\
2.80 \mathrm{E}-08 \\
2.49 \mathrm{E}-08 \\
2.31 \mathrm{E}-08 \\
2.04 \mathrm{E}-08 \\
1.72 \mathrm{E}-08 \\
1.48 \mathrm{E}-08 \\
1.29 \mathrm{E}-08 \\
1.15 \mathrm{E}-08 \\
1.07 \mathrm{E}-08\end{array}$ & $\begin{array}{l}2.61 \mathrm{E}-07 \\
2.53 \mathrm{E}-07 \\
2.30 \mathrm{E}-07 \\
2.05 \mathrm{E}-07 \\
1.84 \mathrm{E}-07 \\
1.66 \mathrm{E}-07 \\
1.52 \mathrm{E}-07 \\
1.37 \mathrm{E}-07 \\
1.22 \mathrm{E}-07 \\
1.09 \mathrm{E}-07 \\
9.55 \mathrm{E}-08 \\
8.51 \mathrm{E}-08 \\
7.41 \mathrm{E}-08 \\
6.18 \mathrm{E}-08 \\
5.28 \mathrm{E}-08 \\
4.70 \mathrm{E}-08 \\
4.09 \mathrm{E}-08 \\
3.41 \mathrm{E}-08 \\
2.87 \mathrm{E}-08 \\
2.56 \mathrm{E}-08 \\
2.36 \mathrm{E}-08 \\
2.10 \mathrm{E}-08 \\
1.76 \mathrm{E}-08 \\
1.52 \mathrm{E}-08 \\
1.33 \mathrm{E}-08 \\
1.20 \mathrm{E}-08 \\
1.11 \mathrm{E}-08\end{array}$ & $\begin{array}{r}5.94 \\
6.25 \\
6.55 \\
6.83 \\
7.23 \\
7.73 \\
8.25 \\
8.77 \\
9.24 \\
9.72 \\
10.23 \\
10.75 \\
11.26 \\
11.76 \\
12.40 \\
13.20 \\
14.03 \\
14.86 \\
15.62 \\
16.45 \\
17.44 \\
18.51 \\
19.58 \\
20.53 \\
21.43 \\
22.40 \\
23.43\end{array}$ & $\begin{array}{r}9.78 \mathrm{E}-09 \\
8.68 \mathrm{E}-09 \\
7.91 \mathrm{E}-09 \\
7.36 \mathrm{E}-09 \\
6.49 \mathrm{E}-09 \\
5.38 \mathrm{E}-09 \\
4.26 \mathrm{E}-09 \\
3.78 \mathrm{E}-09 \\
3.73 \mathrm{E}-09 \\
3.68 \mathrm{E}-09 \\
3.78 \mathrm{E}-09 \\
3.97 \mathrm{E}-09 \\
4.05 \mathrm{E}-09 \\
4.54 \mathrm{E}-09 \\
7.38 \mathrm{E}-09 \\
1.61 \mathrm{E}-08 \\
2.66 \mathrm{E}-08 \\
2.82 \mathrm{E}-08 \\
2.07 \mathrm{E}-08 \\
1.07 \mathrm{E}-08 \\
3.57 \mathrm{E}-09 \\
7.99 \mathrm{E}-10 \\
1.17 \mathrm{E}-10 \\
-2.53 \mathrm{E}-11 \\
-6.43 \mathrm{E}-11 \\
-7.72 \mathrm{E}-11 \\
-6.94 \mathrm{E}-11-\end{array}$ & $\begin{array}{r}1.01 \mathrm{E}-08 \\
9.09 \mathrm{E}-09 \\
8.34 \mathrm{E}-09 \\
7.73 \mathrm{E}-09 \\
6.83 \mathrm{E}-09 \\
5.78 \mathrm{E}-09 \\
4.61 \mathrm{E}-09 \\
4.08 \mathrm{E}-09 \\
4.05 \mathrm{E}-09 \\
3.97 \mathrm{E}-09 \\
4.07 \mathrm{E}-09 \\
4.29 \mathrm{E}-09 \\
4.37 \mathrm{E}-09 \\
4.85 \mathrm{E}-09 \\
7.69 \mathrm{E}-09 \\
1.63 \mathrm{E}-08 \\
2.68 \mathrm{E}-08 \\
2.84 \mathrm{E}-08 \\
2.09 \mathrm{E}-08 \\
1.08 \mathrm{E}-08 \\
3.62 \mathrm{E}-09 \\
8.23 \mathrm{E}-10 \\
1.38 \mathrm{E}-10 \\
-6.34 \mathrm{E}-12 \\
-4.88 \mathrm{E}-11 \\
-6.04 \mathrm{E}-11 \\
-5.08 \mathrm{E}-11\end{array}$ \\
\hline
\end{tabular}


Table A.19 (Cont'd)

Gamma-Ray Data

Detector 3 in Fig. 5 (shadow bar in place)

Detector Coordinates $(\mathrm{cm}): \quad x=0.0, y=0.0, z^{\prime}=213.0$

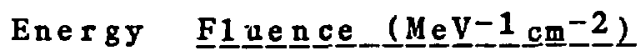
(MeV) Confidence Interval

\begin{tabular}{ll}
\hline 0.72 & $2.73 \mathrm{E}-07$ \\
0.76 & $4.30 \mathrm{E}-07$ \\
0.80 & $5.21 \mathrm{E}-07$ \\
0.84 & $5.31 \mathrm{E}-07$ \\
0.88 & $4.78 \mathrm{E}-07$ \\
0.92 & $3.97 \mathrm{E}-07$ \\
0.96 & $3.21 \mathrm{E}-07$ \\
1.00 & $2.67 \mathrm{E}-07$ \\
1.04 & $2.36 \mathrm{E}-07$ \\
1.08 & $2.23 \mathrm{E}-07$ \\
1.13 & $2.24 \mathrm{E}-07$ \\
1.17 & $2.35 \mathrm{E}-07$ \\
1.22 & $2.41 \mathrm{E}-07$ \\
1.28 & $2.33 \mathrm{E}-07$ \\
1.33 & $2.12 \mathrm{E}-07$ \\
1.38 & $1.86 \mathrm{E}-07$ \\
1.42 & $1.60 \mathrm{E}-07$ \\
1.47 & $1.39 \mathrm{E}-07$ \\
1.53 & $1.25 \mathrm{E}-07$ \\
1.58 & $1.18 \mathrm{E}-07$ \\
1.64 & $1.17 \mathrm{E}-07$ \\
1.72 & $1.20 \mathrm{E}-07$ \\
1.80 & $1.24 \mathrm{E}-07$ \\
1.88 & $1.25 \mathrm{E}-07$ \\
1.96 & $1.23 \mathrm{E}-07$ \\
2.04 & $1.19 \mathrm{E}-07$ \\
2.12 & $1.14 \mathrm{E}-07$ \\
2.20 & $1.08 \mathrm{E}-07$ \\
2.28 & $9.87 \mathrm{E}-08$ \\
2.36 & $8.77 \mathrm{E}-08$ \\
2.45 & $7.55 \mathrm{E}-08$ \\
2.55 & $6.56 \mathrm{E}-08$ \\
2.65 & $6.02 \mathrm{E}-08$ \\
2.75 & $5.66 \mathrm{E}-08$ \\
2.85 & $5.23 \mathrm{E}-08$ \\
2.95 & $4.74 \mathrm{E}-08$ \\
3.05 & $2.37 \mathrm{E}-08$ \\
3.15 & $4.19 \mathrm{E}-08$ \\
3.25 & $4.16 \mathrm{E}-08$ \\
3.35 & $4.23 \mathrm{E}-08$ \\
3.45 & $4.34 \mathrm{E}-08$ \\
3.55 & $4.40 \mathrm{E}-08$ \\
3.66 & $4.27 \mathrm{E}-08$ \\
3.79 & $3.85 \mathrm{E}-08$
\end{tabular}

$4.20 \mathrm{E}-07$

$4.89 \mathrm{E}-07$

$5.39 \mathrm{E}-07$

$5.38 \mathrm{E}-07$

$4.83 \mathrm{E}-07$

$4.02 \mathrm{E}-07$

$3.26 \mathrm{E}-07$

$2.71 \mathrm{E}-07$

$2.39 \mathrm{E}-07$

$2.26 \mathrm{E}-07$

$2.27 \mathrm{E}-07$

$2.38 \mathrm{E}-07$

$2.45 \mathrm{E}-07$

$2.37 \mathrm{E}-07$

$2.16 \mathrm{E}-0.7$

$1.89 \mathrm{E}-07$

$1.63 \mathrm{E}-07$

$1.42 \mathrm{E}-07$

$1.28 \mathrm{E}-07$

$1.21 \mathrm{E}-07$

$1.19 \mathrm{E}-07$

$1.22 \mathrm{E}-07$

$1.26 \mathrm{E}-07$

$1.28 \mathrm{E}-07$

$1.26 \mathrm{E}-07$

1. $21 \mathrm{E}-07$

$1.16 \mathrm{E}-07$

1. $10 \mathrm{E}-07$

$1.01 \mathrm{E}-07$

$8.96 \mathrm{E}-08$

$7.74 \mathrm{E}-08$

$6.74 \mathrm{E}-08$

$6.20 \mathrm{E}-08$

$5.84 \mathrm{E}-08$

$5.40 \mathrm{E}-08$

$4.91 \mathrm{E}-08$

$4.54 \mathrm{E}-08$

$4.37 \mathrm{E}-08$

$4.35 \mathrm{E}-08$

$4.41 \mathrm{E}-08$

$4.52 \mathrm{E}-08$

$4.59 \mathrm{E}-08$

$4.06 \mathrm{E}-08$
$4.47 \mathrm{E}-08$

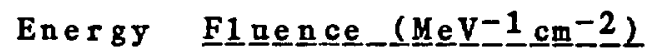
(MeV) Confidence Interval

3.93

4.06

4.19

4.31

4.44

4.57

4.70

4.83

4.99

5.15

5.32

5.50

5.67

5.83

6.00

6.18

6.35

6.51

6.70

6.90

7.10

7.30

7.50

7.70

7.90

8.10

8.30

8.50

8.73

8.98

9.23

9.48

9.73

9.98

10.30

10.70

11.10

11.50

11.90

12.30

12.70

13.10

13.50

13.90
$3.32 \mathrm{E}-08$

2.88 E- 08

2. $59 \mathrm{E}-08$

2. 46 E-0 8

2.54 E-08

2.81 E- 08

$3.09 \mathrm{E}-08$

$3.13 \mathrm{E}-08$

2. $76 \mathrm{E}-08$

2.02 E- 08

1.44 E-0 8

$1.39 \mathrm{E}-08$

$1.96 \mathrm{E}-08$

$2.77 \mathrm{E}-08$

$3.30 \mathrm{E}-08$

$3.34 \mathrm{E}-08$

$2.99 \mathrm{E}-08$

$2.54 \mathrm{E}-08$

$2.19 \mathrm{E}-08$

$2.05 \mathrm{E}-08$

$2.08 \mathrm{E}-08$

$2.09 \mathrm{E}-08$

$1.99 \mathrm{E}-08$

1.72 E- 08

$1.36 \mathrm{E}-08$

$9.80 \mathrm{E}-09$

$6.80 \mathrm{E}-09$

$4.87 \mathrm{E}-09$

$3.75 \mathrm{E}-09$

3.19E-09

2.81E-09

$2.27 \mathrm{E}-09$

1. $51 \mathrm{E}-09$

$6.80 \mathrm{E}-10$

$-1.72 E-10$

$-5.98 E-10$

$-4.08 \mathrm{E}-10$

$-1.56 \mathrm{E}-10$

-8.44 E-11

$-3.07 E-11$

1. $65 \mathrm{E}-12$

$-2.94 E-11$

$-5.08 E-11$

-4.78 E- 11
3. $53 \mathrm{E}-08$
$3.11 \mathrm{E}-08$
$2.83 \mathrm{E}-08$
2.74 E- 08
$2.87 \mathrm{E}-08$
$3.16 \mathrm{E}-08$
3.44 E- 08
3. $50 \mathrm{E}-08$
$3.21 \mathrm{E}-08$
2. $50 \mathrm{E}-08$
1.92 E-0 8
$1.92 \mathrm{E}-08$
2. 52 E-0 8
3. $30 \mathrm{E}-08$
$3.78 \mathrm{E}-08$
$3.82 \mathrm{E}-08$
$3.45 \mathrm{E}-08$
2.94 E-0 8
2.52 E- 08
$2.35 \mathrm{E}-08$
$2.37 \mathrm{E}-08$
2.36 E- 08
$2.24 \mathrm{E}-0.8$
$1.95 \mathrm{E}-08$
$1.57 \mathrm{E}-08$
1.17 E-0 8
8.47 E-09
$6.39 \mathrm{E}-09$
$5.08 \mathrm{E}-09$
$4.41 \mathrm{E}-09$
3.84 E- 09
$3.19 \mathrm{E}-09$
$2.45 \mathrm{E}-09$
$1.73 \mathrm{E}-09$
$1.01 \mathrm{E}-09$
$6.10 \mathrm{E}-10$
$5.08 \mathrm{E}-10$
$5.83 \mathrm{E}-10$
$8.20 E-10$
8. 22 E-10
$6.89 \mathrm{E}-10$
$5.65 \mathrm{E}-10$
$4.60 \mathrm{E}-10$
$3.75 \mathrm{E}-10$ 
Table A. 20

Neutron Data

Detector 4 in Fig. 5 (shadow bar in place)

Detector Coordinates $(\mathrm{cm}): x=70.0, y=0.0, z^{\prime}=151.0$

\begin{tabular}{|c|c|c|c|c|c|}
\hline \multirow{2}{*}{$\begin{array}{l}\text { Energy } \\
(\mathrm{MeV})\end{array}$} & \multicolumn{2}{|c|}{ 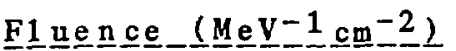 } & \multirow{2}{*}{$\begin{array}{l}\text { Energy } \\
(\mathrm{MeV})\end{array}$} & \multicolumn{2}{|c|}{ 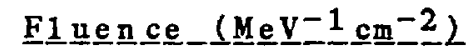 } \\
\hline & Confidence & Interva 1 & & Confidence & Interval \\
\hline $0.9 \overline{1}$ & $2.57 \mathrm{E}-07$ & $2.59 \mathrm{E}-07$ & 5.94 & $1.02 \mathrm{E}-08$ & $1.07 \mathrm{E}-08$ \\
\hline 1.01 & $2.48 \mathrm{E}-07$ & $2.49 \mathrm{E}-07$ & 6.25 & $9.30 \mathrm{E}-09$ & $9.83 \mathrm{E}-09$ \\
\hline 1.11 & $2.21 \mathrm{E}-07$ & $2.23 \mathrm{E}-07$ & 6.55 & $8.82 \mathrm{E}-09$ & $9.37 \mathrm{E}-09$ \\
\hline 1.21 & $1.97 \mathrm{E}-07$ & $1.99 \mathrm{E}-07$ & 6.83 & $8.50 \mathrm{E}-09$ & $8.97 \mathrm{E}-09$ \\
\hline 1.31 & $1.81 \mathrm{E}-07$ & $1.82 \mathrm{E}-07$ & 7.23 & $7.63 \mathrm{E}-09$ & $8.07 \mathrm{E}-09$ \\
\hline 1.41 & $1.68 \mathrm{E}-07$ & $1.69 \mathrm{E}-07$ & 7.73 & $5.84 \mathrm{E}-09$ & $6.35 \mathrm{E}-09$ \\
\hline 1.51 & $1.55 \mathrm{E}-07$ & $1.57 \mathrm{E}-07$ & 8.25 & $3.96 \mathrm{E}-09$ & $4.42 \mathrm{E}-09$ \\
\hline 1.61 & $1.39 \mathrm{E}-07$ & $1.40 \mathrm{E}-07$ & 8.77 & $3.57 \mathrm{E}-09$ & $3.97 \mathrm{E}-09$ \\
\hline 1.71 & $1.21 \mathrm{E}-07$ & $1.22 \mathrm{E}-07$ & 9.24 & $3.59 \mathrm{E}-09$ & $4.02 \mathrm{E}-09$ \\
\hline 1.81 & $1.05 \mathrm{E}-07$ & $1.06 \mathrm{E}-07$ & 9.72 & $3.41 \mathrm{E}-09$ & $3.80 \mathrm{E}-09$ \\
\hline 1.94 & $9.13 \mathrm{E}-08$ & $9.27 \mathrm{E}-08$ & 10.23 & $3.64 \mathrm{E}-09$ & $4.05 \mathrm{E}-09$ \\
\hline 2.11 & $8.37 \mathrm{E}-08$ & $8.46 \mathrm{E}-08$ & 10.75 & $4.21 \mathrm{E}-09$ & $4.66 \mathrm{E}-09$ \\
\hline 2.31 & $7.24 E-08$ & $7.34 \mathrm{E}-08$ & 11.26 & $4.34 \mathrm{E}-09$ & $4.78 \mathrm{E}-09$ \\
\hline 2.50 & $5.86 \mathrm{E}-08$ & $5.94 \mathrm{E}-08$ & 11.76 & $4.73 E-09$ & $5.17 \mathrm{E}-09$ \\
\hline 2.69 & $5.03 \mathrm{E}-08$ & $5.09 \mathrm{E}-08$ & 12.40 & $9.03 \mathrm{E}-09$ & $9.48 \mathrm{E}-09$ \\
\hline 2.90 & $4.74 E-08$ & $4.81 \mathrm{E}-08$ & 13.20 & $2.62 E-08$ & $2.66 \mathrm{E}-08$ \\
\hline 3.10 & $4.24 \mathrm{E}-08$ & $4.34 E-08$ & 14.03 & $5.10 \mathrm{E}-08$ & $5.14 \mathrm{E}-08$ \\
\hline 3.30 & $3.46 \mathrm{E}-08$ & $3.53 \mathrm{E}-08$ & 14.86 & $5.85 \mathrm{E}-08$ & $5.88 \mathrm{E}-08$ \\
\hline 3.51 & $2.76 \mathrm{E}-08$ & $2.85 \mathrm{E}-08$ & 15.62 & $4.49 \mathrm{E}-08$ & $4.52 \mathrm{E}-08$ \\
\hline 3.71 & $2.44 \mathrm{E}-08$ & $2.54 \mathrm{E}-08$ & 16.45 & $2.40 \mathrm{E}-08$ & $2.42 E-08$ \\
\hline 3.90 & $2.35 \mathrm{E}-08$ & $2.41 \mathrm{E}-08$ & 17.44 & $8.30 \mathrm{E}-09$ & $8.38 \mathrm{E}-09$ \\
\hline 4.15 & $2.13 \mathrm{E}-08$ & $2.20 \mathrm{E}-08$ & 18.51 & $1.92 \mathrm{E}-09$ & $1.96 \mathrm{E}-09$ \\
\hline 4.46 & $1.75 \mathrm{E}-08$ & $1.79 \mathrm{E}-08$ & 19.58 & $3.02 \mathrm{E}-10$ & $3.37 \mathrm{E}-10$ \\
\hline 4.75 & $1.50 \mathrm{E}-08$ & $1.55 \mathrm{E}-08$ & 20.53 & $-3.57 \mathrm{E}-11$ & $-5.09 \mathrm{E}-12$ \\
\hline 5.06 & $1.33 \mathrm{E}-08$ & $1.38 \mathrm{E}-08$ & 21.43 & $-1.26 E-10$ & $-1.01 \mathrm{E}-10$ \\
\hline 5.36 & $1.17 \mathrm{E}-08$ & $1.23 \mathrm{E}-08$ & 22.40 & $-1.54 E-10$ & $-1.27 E-10$ \\
\hline 5.64 & $1.09 \mathrm{E}-08$ & $1.15 \mathrm{E}-08$ & 23.43 & $-1.39 E-10$ & $-1.09 \mathrm{E}-10$ \\
\hline
\end{tabular}




\section{Table A.20 (Cont'd)}

Gamma-Ray Data

Detector 4 in Fig. 5 (shadow bar in place)

Detector Coordinates $(\mathrm{cm}): x=79.0, y=0.0, z^{\prime}=151.0$

\begin{tabular}{|c|c|c|c|c|c|}
\hline \multirow{2}{*}{$\begin{array}{l}\text { Energy } \\
(\mathrm{MeV})\end{array}$} & \multicolumn{2}{|c|}{$\underline{F} \underline{\underline{u}} \underline{\mathrm{e}} \underline{\mathrm{c}} \mathrm{e} \underline{\mathrm{e}}\left(\underline{\mathrm{Me}} \underline{\mathrm{V}}-1 \mathrm{c} \mathrm{m}^{-2}\right)$} & \multirow{2}{*}{$\begin{array}{l}\text { Energy } \\
(\mathrm{MeV})\end{array}$} & \multicolumn{2}{|c|}{ 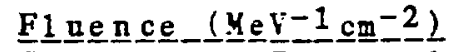 } \\
\hline & Confidence & Interva 1 & & & Interva 1 \\
\hline 0.72 & $2.49 \mathrm{E}-07$ & $3.96 \mathrm{E}-07$ & 3.93 & $3.40 \mathrm{E}-08$ & $3.62 \mathrm{E}-08$ \\
\hline 0.76 & $4.09 \mathrm{E}-07$ & $4.69 \mathrm{E}-07$ & 4.06 & $3.08 \mathrm{E}-08$ & $3.33 \mathrm{E}-08$ \\
\hline 0.80 & $4.94 E-07$ & $5.11 \mathrm{E}-07$ & 4.19 & $2.84 \mathrm{E}-08$ & $3.09 \mathrm{E}-08$ \\
\hline 0.84 & $5.00 \mathrm{E}-07$ & $5.07 \mathrm{E}-07$ & 4.31 & $2.68 \mathrm{E}-08$ & $2.98 \mathrm{E}-08$ \\
\hline 0.88 & $4.53 \mathrm{E}-07$ & $4.59 \mathrm{E}-07$ & 4.44 & $2.72 \mathrm{E}-08$ & $3.07 \mathrm{E}-08$ \\
\hline 0.92 & $3.83 \mathrm{E}-07$ & $3.89 \mathrm{E}-07$ & 4.57 & $2.97 \mathrm{E}-08$ & $3.35 \mathrm{E}-08$ \\
\hline 0.96 & $3.17 \mathrm{E}-07$ & $3.22 \mathrm{E}-07$ & 4.70 & $3.29 \mathrm{E}-08$ & $3.67 \mathrm{E}-08$ \\
\hline 1.00 & $2.69 \mathrm{E}-07$ & $2.73 E-07$ & 4.83 & $3.39 \mathrm{E}-08$ & $3.78 \mathrm{E}-08$ \\
\hline 1.04 & $2.41 \mathrm{E}-07$ & $2.45 \mathrm{E}-07$ & 4.99 & $3.06 \mathrm{E}-08$ & $3.55 \mathrm{E}-08$ \\
\hline 1.08 & $2.31 \mathrm{E}-07$ & $2.34 \mathrm{E}-07$ & 5.15 & $2.35 \mathrm{E}-08$ & $2.86 \mathrm{E}-08$ \\
\hline 1.13 & $2.33 \mathrm{E}-07$ & $2.36 \mathrm{E}-07$ & 5.32 & $1.75 \mathrm{E}-08$ & $2.26 \mathrm{E}-08$ \\
\hline 1.17 & $2.41 \mathrm{E}-07$ & $2.44 \mathrm{E}-07$ & 5.50 & $1.52 \mathrm{E}-08$ & $2.09 \mathrm{E}-08$ \\
\hline 1.22 & $2.42 \mathrm{E}-07$ & $2.46 \mathrm{E}-07$ & 5.67 & $1.82 \mathrm{E}-08$ & $2.42 \mathrm{E}-08$ \\
\hline 1.28 & $2.32 \mathrm{E}-07$ & $2.36 \mathrm{E}-07$ & 5.83 & $2.47 \mathrm{E}-08$ & $3.04 \mathrm{E}-08$ \\
\hline 1.33 & $2.13 \mathrm{E}-07$ & $2.16 \mathrm{E}-07$ & 6.00 & $3.17 \mathrm{E}-08$ & $3.69 \mathrm{E}-08$ \\
\hline 1.38 & $1.90 \mathrm{E}-07$ & $1.93 E-07$ & 6.18 & $3.58 \mathrm{E}-08$ & $4.10 \mathrm{E}-08$ \\
\hline 1.42 & $1.68 \mathrm{E}-07$ & $1.72 \mathrm{E}-07$ & 6.35 & $3.53 \mathrm{E}-$ & $4.02 \mathrm{E}-08$ \\
\hline 1.47 & $1.49 \mathrm{E}-07$ & $1.52 \mathrm{E}-07$ & 6.51 & $3.10 \mathrm{E}-$ & $3.53 \mathrm{E}-08$ \\
\hline 1.53 & $1.34 \mathrm{E}-07$ & $1.37 \mathrm{E}-07$ & 6.70 & $2.48 \mathrm{E}-08$ & $2.83 E-08$ \\
\hline 1.58 & $1.25 \mathrm{E}-07$ & $1.28 \mathrm{E}-07$ & 6.90 & $1.95 \mathrm{E}-08$ & $2.27 \mathrm{E}-0.8$ \\
\hline 1.64 & $1.23 \mathrm{E}-07$ & $1.25 \mathrm{E}-07$ & 7.10 & $1.78 \mathrm{E}-08$ & $2.08 \mathrm{E}-08$ \\
\hline 1.72 & $1.26 \mathrm{E}-07$ & $1.29 \mathrm{E}-07$ & 7.30 & $1.88 \mathrm{E}-08$ & $2.16 \mathrm{E}-08$ \\
\hline 1.80 & $1.30 \mathrm{E}-07$ & $1.33 \mathrm{E}-07$ & 7.50 & $2.01 \mathrm{E}-08$ & $2.27 E-08$ \\
\hline 1.88 & $1.34 \mathrm{E}-07$ & $1.36 \mathrm{E}-07$ & 7.70 & $1.93 \mathrm{E}-08$ & $2.18 E-08$ \\
\hline 1.96 & $1.34 \mathrm{E}-07$ & $1.36 \mathrm{E}-07$ & 7.90 & $1.62 \mathrm{E}-08$ & $1.84 \mathrm{E}-08$ \\
\hline 2.04 & $1.29 \mathrm{E}-07$ & $1.32 \mathrm{E}-07$ & 8.10 & $1.17 \mathrm{E}-08$ & $1.37 \mathrm{E}-08$ \\
\hline 2.12 & $1.23 \mathrm{E}-07$ & $1.25 \mathrm{E}-07$ & 8.30 & $7.32 \mathrm{E}-09$ & $9.12 \mathrm{E}-09$ \\
\hline 2.20 & $1.17 \mathrm{E}-07$ & $1.19 \mathrm{E}-07$ & 8.50 & $4.29 \mathrm{E}-09$ & $5.90 \mathrm{E}-09$ \\
\hline 2.28 & $1.07 \mathrm{E}-07$ & $1.09 \mathrm{E}-07$ & 8.73 & $2.76 E-09$ & $4.16 \mathrm{E}-09$ \\
\hline 2.36 & $9.34 \mathrm{E}-08$ & $9.54 \mathrm{E}-08$ & 8.98 & $2.78 \mathrm{E}-09$ & $4.02 \mathrm{E}-09$ \\
\hline 2.45 & $7.85 \mathrm{E}-08$ & $8.04 \mathrm{E}-08$ & 9.23 & $3.31 \mathrm{E}-09$ & $4.40 \mathrm{E}-09$ \\
\hline 2.55 & $6.89 E-08$ & $7.08 \mathrm{E}-08$ & 9.48 & $3.31 \mathrm{E}-09$ & $4.26 \mathrm{E}-09$ \\
\hline 2.65 & $6.35 \mathrm{E}-08$ & $6.54 \mathrm{E}-08$ & 9.73 & $2.55 \mathrm{E}-09$ & $3.37 \mathrm{E}-09$ \\
\hline 2.75 & $5.80 E-08$ & $5.99 \mathrm{E}-08$ & 9.98 & $1.41 \mathrm{E}-09$ & $2.13 \mathrm{E}-09$ \\
\hline 2.85 & $5.29 \mathrm{E}-08$ & $5.48 \mathrm{E}-08$ & 10.30 & $1.23 \mathrm{E}-10$ & $7.90 \mathrm{E}-10$ \\
\hline 2.95 & $4.98 E-08$ & $5.16 \mathrm{E}-08$ & 10.70 & $-5.80 E-10$ & $9.88 \mathrm{E}-11$ \\
\hline 3.05 & $4.79 \mathrm{E}-08$ & $4.97 \mathrm{E}-08$ & 11.10 & $-5.88 E-10$ & $9.89 \mathrm{E}-11$ \\
\hline 3.15 & $4.59 \mathrm{E}-08$ & $4.78 \mathrm{E}-08$ & 11.50 & $-3.46 E-10$ & $2.69 \mathrm{E}-10$ \\
\hline 3.25 & $4.44 \mathrm{E}-08$ & $4.64 \mathrm{E}-08$ & 11.90 & $-6.36 E-11$ & $3.96 \mathrm{E}-10$ \\
\hline 3.35 & $4.40 \mathrm{E}-08$ & $4.59 E-08$ & 12.30 & $1.21 \mathrm{E}-10$ & $4.80 \mathrm{E}-10$ \\
\hline 3.45 & $4.43 E-08$ & $4.62 \mathrm{E}-08$ & 12.70 & $2.09 \mathrm{E}-10$ & $5.46 \mathrm{E}-10$ \\
\hline 3.55 & $4.41 \mathrm{E}-0 \mathrm{~s}$ & $4.61 \mathrm{E}-08$ & 13.10 & $2.22 \mathrm{E}-10$ & $5.94 \mathrm{E}-10$ \\
\hline 3.66 & $4.20 E-08$ & $4.42 \mathrm{E}-08$ & 13.50 & $1.29 \mathrm{E}-10$ & $5.19 \mathrm{E}-10$ \\
\hline 3.79 & $3.80 E-08$ & $4.02 \mathrm{E}-08$ & 13.90 & $6.05 E-11$ & $3.72 \mathrm{E}-10$ \\
\hline
\end{tabular}

